\title{
Efficient Hermite-based Variability Analysis using Decoupling Technique
}

\author{
by
}

\author{
Tuan Anh Pham, B.Eng
}

\begin{abstract}
A Thesis submitted to the Faculty of Graduate Studies and Postdoctoral Affairs in partial fulfilment of the requirements for the degree of

Master of Applied Science

in
\end{abstract}

Electrical and Computer Engineering

\author{
Ottawa-Carleton Institute for Electrical and Computer Engineering \\ Department of Electronics \\ Faculty of Engineering \\ Carleton University \\ Ottawa, Ontario, Canada \\ January 2014
}

Copyright (C) 2014

Tuan Anh Pham 


\section{Abstract}

During the design and fabrication process of electronic systems, one of the major concerns is predicting the effect of the variability of their geometrical and physical parameters on the general performance of the designed circuits.

To address the above difficulty, this thesis presents a new Hermite-based approach to circuit variability analysis using the Polynomial-Chaos (PC) paradigm. The new approach is aimed at limiting the growth of the computational cost of variability analysis with the increase in the number of random variables and the number of Hermite coefficients used to represent the circuit response in each random variable. The proposed method is based on deriving a closed-form for the structure of the augmented matrices generated by the PC approach. An algorithm is then developed to decouple the large augmented matrices into independent matrices that can be factorized in parallel. In addition, the model-order reduction (MOR) is applied to the circuit stochastic analysis using the proposed PC approach to show its ability to achieve an additional speed-up while still retaining the result accuracy. Several numerical examples are presented to demonstrate the computational efficiency of the proposed method and its ability to handle the increasing dimension of random space. 


\section{Acknowledgments}

I would like to express my deep appreciation to my co-supervisors, Prof. Michel Nakhla, Prof. Emad Gad and Prof. Ram Achar, for their constant support and encouragement, as well as their valuable advices which made this work one of the most memorable experiences in my life. I am truly grateful to Professor Nakhla for all his thorough guidance and support since the first day I came to Carleton University. I would also like to thank Prof. Emad Gad for introducing me to the fascinating world of circuit variability analysis and giving me a chance to do a research on this interesting topic.

I also acknowledge my colleagues in our CAD group, especially Mina and Behzad, for their friendly and valuable discussions which helped me a lot, not only in this work, but also in my graduate life here in Carleton. Specially, I really appreciate Mina for his very kind help in $\mathrm{C}++$.

I would like to give special thanks to my fiancee, Jolie, for all her support, understanding and respect, which motivates me a lot to overcome all the difficulties and obstacles. Above all, her love is the greatest encouragement to me when I live far away from my hometown.

Last but not least, I would like to express my deepest gratitude to my family, especially my mother, for her unconditional love and caring, and my father, who always follows every steps of mine, I believe, from heaven. To them, I dedicate this thesis. 


\section{Table of Contents}

Abstract $\quad$ ii

Acknowledgments $\quad$ iii

Table of Contents $\quad$ iv

List of Tables vii

List of Figures viii

List of Acronyms $\quad$ X

List of Symbols $\quad$ xii

1 Introduction 1

1.1 Background and Motivation . . . . . . . . . . . . . . 1

1.2 Objectives and Contributions ................... 3

1.3 Organization of the Thesis . . . . . . . . . . . . . 4

2 Background on Stochastic Analysis 5

2.1 Probabilistic and Statistical Background . . . . . . . . . . 6

2.2 The Monte-Carlo Method . . . . . . . . . . . . . 7

2.2.1 Basic knowledge of MC method . . . . . . . . . . . . . . 7

2.2.2 Sample Mean Estimator . . . . . . . . . . . . . . . . . . . . 9 
2.2.3 Sample Standard Deviation Estimator . . . . . . . . . . . . . 9

2.3 Polynomial Chaos Paradigm . . . . . . . . . . . . . . . . 10

2.3.1 Hermite Polynomial Chaos for Single Random Variable . . . . 11

2.3.2 Hermite Polynomial Chaos for Multi-random Variables . . . . 14

2.3.3 Truncation Schemes . . . . . . . . . . . . . 15

2.3.4 Ordering the Multi-indices . . . . . . . . . . . . . . . . 16

3 Applications of Polynomial-Chaos to Circuit Variability Analysis 20

3.1 Frequency Domain Variability Analysis . . . . . . . . . . . . . 20

3.2 Illustrative Example of the Augmented System . . . . . . . . . . . . . 25

3.3 Difficulties with the PC Approach . . . . . . . . . . . . 26

4 Closed-form Representation of Augmented Matrices arising from $\begin{array}{ll}\text { Hermite-based PC } & 27\end{array}$

4.1 The Case of a Single Random Variable . . . . . . . . . . . . . . . . . 27

4.1 Lemma 1 . . . . . . . . . . . . . . . . . . . . . . 28

4.1 .2 Lemma 2 . . . . . . . . . . . . . . . . . . . . . 32

4.1 .3 Lemma 3 . . . . . . . . . . . . . . . . 36

4.2 The Case of Multi-random Variables . . . . . . . . . . . . . . 36

4.3 Closed-form Representation of Augmented Matrices using Total Order 39

5 The Proposed Decoupling Approach $\quad 41$

5.1 The Case of a Single Random Parameter . . . . . . . . . . . . . . . . 41

5.1.1 The Selector Operator . . . . . . . . . . . . . . 42

5.1.2 Approximate Decoupling Technique . . . . . . . . . . . 43

5.2 The Case of Multi-random Parameters . . . . . . . . . . . . . . 46

5.3 An Improved Decoupled Formulation . . . . . . . . . . . . . . . . . . 46

5.4 Numerical Validation . . . . . . . . . . . . . . . . . 50 
5.4.1 Example 1.................... 50

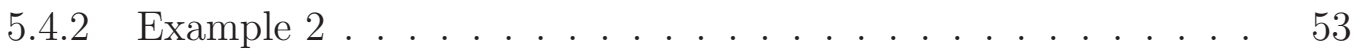

5.4 .3 Example $3 \ldots \ldots \ldots$. . . . . . . . . . . 57

5.4.4 The CPU Time Comparison . . . . . . . . . . . . . . 61

6 Application of Model Order Reduction to Variability Analysis 63

6.1 Applying MOR to Circuit Variability Analysis using Standard PC Approach ............................ 65

6.2 Applying MOR to Circuit Variability Analysis using Decoupled PC

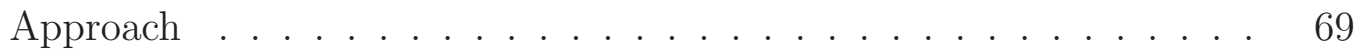

6.3 Numerical Example . . . . . . . . . . . . . . . . . . . . . . 73

7 Conclusions and Future Work $\quad 78$

7.1 Conclusions . . . . . . . . . . . . . . . . . . . . . . . 78

7.2 Future Work . . . . . . . . . . . . . . . . . . . . 79

$\begin{array}{ll}\text { List of References } & 81\end{array}$ 


\section{List of Tables}

1 Wiener-Askey polynomial chaos and their corresponding optimal random variables . . . . . . . . . . . . . . . . . . . 11

2 Hermite polynomials of orders up to $M=4 \ldots \ldots$. . . . . 13

3 An example of ordering $\boldsymbol{\alpha}$ using total order where $d=2$ and $M=2 . \quad 15$

4 An example of ordering $\boldsymbol{\alpha}$ using tensor order where $d=2$ and $M=2 \quad 17$

5 An example of ordering $\boldsymbol{\alpha}$ using total lexicographic order where $d=2$ and $M=2 \ldots \ldots \ldots \ldots \ldots$

6 Problems sizes and CPU computation time (CPU time for experiments that could not be completed due to lack of memory are marked with

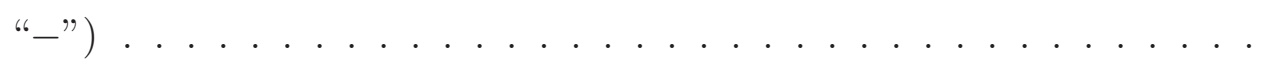

7 CPU computation time with and without MOR (speed-ups are obtained compared with the case of coupled PC without MOR) . . . . 


\section{List of Figures}

1 Standard deviation diagram $\ldots \ldots \ldots \ldots \ldots \ldots$

2 Two coupled transmission-line circuit (top panel) and its cross-section (bottom panel) of Example 1. . . . . . . . . . . . . . . . . 51

3 Sparsity patterns of augmented coupled matrix $\overline{\boldsymbol{G}}^{*}$ with total order (left hand side) and augmented decoupled matrix $\check{G}$ with tensor order (right hand side $) \ldots \ldots \ldots \ldots \ldots$. . . . . . . . . . . . . 51

4 Statistical analysis for the magnitude of the far-end voltage of line 1 (example 1) . . . . . . . . . . . . . . . . . . . 52

5 Statistical analysis for the magnitude of the far-end cross-talk of line 2 (example 1) . . . . . . . . . . . . . . .

6 Eight coupled transmission-line circuit (top panel) and its cross-section (bottom panel) of example $2 \ldots \ldots \ldots \ldots$

7 Statistical analysis for the magnitude of the far-end voltage of line 1

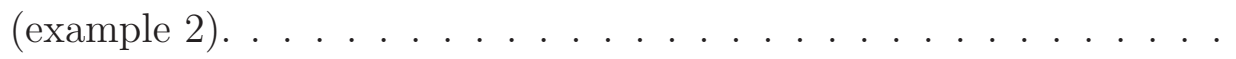

8 Statistical analysis for the magnitude of the far-end voltage of line 3

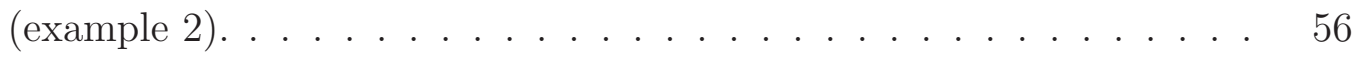

9 Statistical analysis for the magnitude of the far-end voltage of line 5

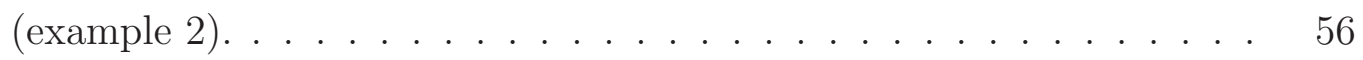

10 Statistical analysis for the magnitude of the far-end voltage of line 7

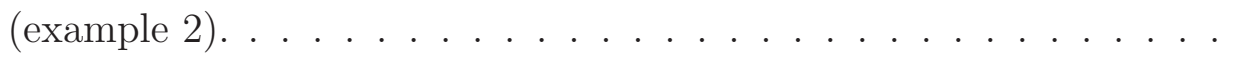


11 Schematic of an active low-pass filter. . . . . . . . . . . . 58

12 Op-Amp subcircuit model. . . . . . . . . . . . . . . . 58

13 Pass-band response of the filter (Example 3) . . . . . . . . . . . 59

14 Stop-band response of the filter (Example 3) . . . . . . . . . . . . 59

15 Yield of the Filter. . . . . . . . . . . . . . . . . . 60

16 Circuit of three 32-MTL groups and the cross-section of each MTL group (bottom left of the figure) . . . . . . . . . . . . 73

17 Statistical analysis for the magnitude of the far-end voltage of line 2-1. 75

18 Statistical analysis for the magnitude of the far-end voltage of line 2-9. 75

19 Statistical analysis for the magnitude of the far-end voltage of line 2-17. 76

20 Statistical analysis for the magnitude of the far-end voltage of line 2-25. 76 


\section{List of Acronyms}

\begin{tabular}{ll} 
Acronyms & Definition \\
\hline \hline AWE & Asymptotic Waveform Evaluation \\
CAD & Computer Aided Design \\
CAL & Computer Aided Learning \\
CPU & central processing unit \\
DAE & Differential-Algebraic Equations \\
EM & Electro-Magnetic \\
gPC & generalized Polynomial Chaos \\
IC & Integrated Circuit \\
LPF & Low Pass Filter \\
MATLAB & Matrix Laboratory Software \\
MC & Monte Carlo \\
MNA & Modified Nodal Analysis \\
MMTs & Moment Matching Techniques \\
\hline
\end{tabular}




\begin{tabular}{ll} 
MOR & Model Order Reduction \\
MTL & Multi-conductor Transmission Line \\
PC & Polynomial Chaos \\
PDF & Probability Density Function \\
PRIMA & Passive Reduced-order Interconnect Macro-modeling Algorithm \\
p.u.l. & Per unit length \\
RF & Radio Frequency \\
SPICE & Simulation Program with Integrated Circuit Emphasis \\
TL & Transmission Line \\
VLSI & Very Large-Scale Integration \\
\hline
\end{tabular}




\title{
List of Symbols
}

\author{
Symbols Definition

\begin{tabular}{ll}
\hline \hline$\mu$ & sample mean \\
$\sigma$ & sample standard deviation \\
$v a r$ & sample variance \\
$\xi$ & single random variable \\
$\xi$ & set of multi-random variable \\
$<,>$ & inner product \\
$H_{i}(\xi)$ & Hermite polynomial of degree "i" where $i=0,1, \cdots$ \\
$w(\xi)$ & weighting function \\
$\delta_{i j}$ & Kronecker delta function \\
$X$ & stochastic process of the single random variable $\xi$ \\
$X_{i}$ & a scalar expansion coefficient corresponding to the Hermite \\
& polynomial $H_{i}(\xi)$ \\
$\Omega$ & multi-dimensional random space
\end{tabular}


$\phi_{\boldsymbol{\alpha}}(\boldsymbol{\xi}) \quad$ multi-dimensional polynomials that are orthogonal with respect to a probability measure $W(\boldsymbol{\xi})$

$\boldsymbol{\alpha} \quad$ multi-index

$\boldsymbol{\beta} \quad$ multi-index

$\alpha_{i} \quad i^{\text {th }}$ component of $\boldsymbol{\alpha}$

$N_{\text {total }} \quad$ total number of Hermite coefficients using total order scheme

$N_{\text {tensor }} \quad$ total number of Hermite coefficients using tensor order scheme

$p_{i} \quad$ highest order of Hermite polynomials corresponding to random variable $\xi_{i}$ in tensor order scheme

$|\boldsymbol{\alpha}| \quad$ rank of a multi-index $\boldsymbol{\alpha}$

$\boldsymbol{G}$ matrix describing the memoryless elements in the circuit

C matrix describing the memory elements in the circuit

$\boldsymbol{X}(s) \quad$ vector of the voltages of circuit nodes, currents in the inductors or independent voltage sources

$X(\boldsymbol{\xi}) \quad$ stochastic process that represents the uncertain response of the system due to multi-random variables in $\boldsymbol{\xi}$

$\boldsymbol{U} \quad$ vector representing the independent stimulus of a circuit

$N$ number of the variables in the circuit response

$\mathcal{H}_{\boldsymbol{\alpha}}(\boldsymbol{\xi}) \quad$ generalized Hermite polynomials 
$H_{m}\left(\xi_{i}\right) \quad$ the single-dimensional $m^{\text {th }}$ degree Hermite polynomial

$\hat{\boldsymbol{G}}_{\boldsymbol{\beta}} \quad$ matrix-valued coefficients in the Hermite expansion of $\boldsymbol{G}$

$\hat{\boldsymbol{C}}_{\boldsymbol{\beta}}$ matrix-valued coefficients in the Hermite expansion of $\boldsymbol{C}$

$\overline{\boldsymbol{G}} \quad$ augmented matrix which is constructed from $\hat{\boldsymbol{G}}_{\boldsymbol{\beta}}$

$\overline{\boldsymbol{C}} \quad$ augmented matrix which is constructed from $\hat{\boldsymbol{C}}_{\boldsymbol{\beta}}$

* $\quad$ superscript which implies the total lexicographic order

$\boldsymbol{\tau} \quad$ selector operator to extract the total lexicographic order from tensor order

$\hat{\boldsymbol{X}}(s) \quad$ vector grouping all Hermite coefficients of all variables of the circuit response

$\hat{\boldsymbol{X}}_{i}(s) \quad$ vector grouping all Hermite coefficients of those Hermite polynomials whose rank is equal to $i$, for all the $N$ circuit variables of the MNA formulation

$\top \quad$ the transpose operator

$\otimes \quad$ the Kronecker product operator

$\boldsymbol{A}_{L} \quad$ matrix defined in Lemma 2 - Chapter 4

$\boldsymbol{A}_{U} \quad$ matrix defined in Lemma 2 - Chapter 4

$\boldsymbol{K}_{i} \quad$ matrix defined in Lemma 1 - Chapter 4

$\check{\boldsymbol{K}}_{i} \quad$ matrix which is constructed by $\left(\boldsymbol{A}_{L}+\boldsymbol{A}_{U}\right)^{i}$

$\tilde{\boldsymbol{K}}_{i} \quad$ an improvedly approximate version of $\boldsymbol{K}_{i}$ 


\begin{tabular}{|c|c|}
\hline$\check{G}$ & augmented matrix which is constructed using $\check{\boldsymbol{K}}_{i}$ \\
\hline$\tilde{G}$ & augmented matrix which is constructed using $\tilde{\boldsymbol{K}}_{i}$ \\
\hline$\check{C}$ & augmented matrix which is constructed using $\check{\boldsymbol{K}}_{i}$ \\
\hline$\tilde{C}$ & augmented matrix which is constructed using $\tilde{\boldsymbol{K}}_{i}$ \\
\hline$\psi$ & a selector operator \\
\hline $\boldsymbol{Y}(s)$ & vector of circuit variables of interest \\
\hline$\overline{\boldsymbol{Y}}(s)$ & vector which contains all the Hermite coefficients of circuit \\
\hline & variables of interest \\
\hline $\boldsymbol{V}$ & matrix of eigenvectors of the matrix $\boldsymbol{A}_{L}+\boldsymbol{A}_{U}$ \\
\hline$\lambda$ & $\begin{array}{l}\text { diagonal matrix whose diagonal elements are eigenvalues } \\
\text { of } \boldsymbol{A}_{L}+\boldsymbol{A}_{U}\end{array}$ \\
\hline $\boldsymbol{I}_{N}$ & identity matrix of size $N$ \\
\hline$\left[\boldsymbol{A}_{U}, \boldsymbol{A}_{L}\right]$ & Lie Bracket operation \\
\hline
\end{tabular}




\section{Chapter 1}

\section{Introduction}

\subsection{Background and Motivation}

In the world of today's technology, electronic systems, such as computers and mobile devices, have become essential parts in our daily lives. With the remarkable growth of very large-scale integration (VLSI) technology as well as computer-aided design (CAD) techniques at both chip and packaging level, these electronic systems can operate up to gigahertz frequencies with the rise times in the order of nanosecond level. The ever increasing demand for high-speed applications require these systems to be more compact and have denser layouts while still retaining the accuracy of the circuit performance, therefore, the precision in the design and manufacturing process of such systems becomes a major concern and extremely important nowadays.

During the early phase of a design, simulating the performance of the entire circuit is traditionally carried out on the basis of a deterministic model with well-defined geometrical and physical parameters. However, in practice, one of the main challenges for any designer is to predict accurately how the inherent process variability of these parameters affects the general performance of the circuit design. The lack of predictability arises mainly from the difficulty of controlling the physical and geometrical parameters during the fabrication process. This effectively makes the numerical 
values for those parameters subject to significant uncertainty, which, in turn, produces uncertainty in the electric performance of the circuit, causing significant measurement inaccuracies compared with the simulation results of the design, possibly resulting in redesigning as well as expensive refabrication. Recently, variability analysis, or stochastic analysis, has become available as a powerful means for modelling and simulating complex electronic systems involving random process variations.

Traditionally, Monte Carlo (MC) analysis has been used in commercial circuit and Electromagnetic (EM) simulations for predicting the statistical distribution of the circuit and system performances. However, the slow convergence for MC has become a computational burden, especially in simulating large and complex circuits. This fact has prompted wide interest in exploring alternative approaches to the problem of statistical analysis of electronic circuits.

A recent approach based on the notion of polynomial chaos (PC) was developed and showed great computational advantage over the standard MC-based analysis [1]. This approach has been used in estimating the statistical properties of different types of circuits. Earlier PC approaches focused on characterizing the variability analysis of interconnects [2] and multi-conductor transmission lines in the presence of process variations [3]. Further work addressed the problem of variability in generic multiport linear circuits [4]. It was also applied to handle the problem of variability due to uncertainty in physical parameters of Carbon Nanotube interconnects [5].

The basic idea of the PC approach is to expand the circuit response in a series of the Askey-Wiener type of orthogonal polynomials, e.g, Hermite polynomials. Subsequent to that, a Galerkin projection (GP) process is carried out to construct a deterministic system of equations in the coefficients of the series of Hermite polynomials.

However, one of the main issues that still hinders widening the domain of applications of the PC-based variability analysis to more problems is the issue of the 
computational cost for general problems. This issue arises from the fact that the computational complexity does not scale favourably with the involved number of random variables and the number of Hermite coefficients used with each random variable. The difficulty therein stems from the fact that the Galerkin projection process always results in a system of equations that couples the Hermite coefficients of each random variable to each component of the circuit response. This fact makes the augmented matrix that must be factorized to be significantly larger and denser (i.e., without the desirable sparsity patterns that characterize general circuits).

It is important to stress that working with the large matrix arising from the GP technique is avoidable if one considers the collocation approach to the PC problem, typically known as the Stochastic Collocation (SC) [6]. Indeed, SC can be viewed as a two-level decoupling process: On the first level, the system is analyzed at grid points that sample the random space at a set of predetermined points. On the second level, the solutions obtained for a particular system component, or a group of components, are assembled and used to solve, either using quadrature or linear regression, for the coefficients of the PC expansion of those components. A good study for the SC versus the Stochastic GP-based (SG) paradigms has been presented recently in [7]. As indicated in this study, the main computational bottleneck of the SG is the large matrix which often times becomes cumbersome to handle.

\subsection{Objectives and Contributions}

The goal of this thesis is to address the above difficulty through presenting an efficient method whose computational complexity scales linearly with the growth of the random variables or the number of Hermite coefficients used to represent the components of circuit responses.

A new method has been developed to present a new general formula to characterize 
the structure of the augmented matrices which arise from a Hermite-based PC. It then shows that this structure can be approximated with a different structure which can be decoupled into smaller components and solved independently, or in parallel leading to higher computational efficiency. In contrast to the SC, the proposed decoupling technique is based on the GP and achieves the decoupling through a direct one-level process using predetermined transformation matrices. Some of the results presented in this work, for the special case of a single random parameter have been introduced in [8] by the author. This thesis will present the generalization of those results to problems with multiple random parameters and provides all the necessary proofs. In another contribution, a Model-order reduction (MOR) is applied to the decoupled matrices, thereby enabling more computational savings.

\subsection{Organization of the Thesis}

The rest of this thesis is organized as follows. Chapter 2 reviews briefly the preliminaries of the variability analysis and describes the basic ideas of the Monte-Carlo method as well as the Polynomial Chaos approach. The applications of Polynomial Chaos to circuit variability analysis in the frequency domain is presented in Chapter 3. Chapter 4 presents new theoretical results to characterize the general structure of the augmented matrices obtained from a Hermite-based PC application to circuit equations. Following to this, the proposed decoupling technique along with several numerical examples to validate its accuracy are described in Chapter 5. Next, Chapter 6 presents the application of MOR to the circuit stochastic analysis using both the standard and the proposed PC approaches. Finally, Chapter 7 provides the concluding remarks and future research options. 


\section{Chapter 2}

\section{Background on Stochastic Analysis}

Stochastic analysis, or variability analysis, is well-known as an effective tool in modeling complex physical and engineering problems involving uncertainty. Specially in circuit simulation at the early design stage of electronic systems, when the circuit parameters are not purely deterministic and suffer from different sources of variations, the stochastic simulation could help designers to predict the performance errors due to uncertainty in manufacturing process and avoid the need for expensive refabrication. There are several ways to predict the impact of the uncertainty in the design parameters on the variability of the circuit performance. The most popular and simplest one is the so-called Monte Carlo method which requires a vast number of simulations with difference samples of random variables to compute the output statistics. Conversely, in the polynomial chaos approach which has been recently proposed and developed, the stochastic process is expressed as a truncated series and only one simulation is required to obtain the statistical behavior of the circuit.

This chapter starts by first outlining, in Section 2.1 some of the fundamental concepts related to probabilistic arguments and random variables. Section 2.2 then presents the basic MC approach and its application in the statistical characterization of the circuit performance. Next, section 2.3 presents the general ideas of the polynomial chaos. 


\subsection{Probabilistic and Statistical Background}

Let $\boldsymbol{\eta}$ be a random variable, which is the stochastic selection of possible values $\eta_{1}, \eta_{2}, \cdots, \eta_{n}$. If the probability of choosing $\eta_{i}$ is given by $P_{i}=\boldsymbol{P}\left\{\boldsymbol{\eta}=\eta_{i}\right\}$, the random variable $\boldsymbol{\eta}$ has its expectation $E(\boldsymbol{\eta})$ which is defined as:

$$
E(\boldsymbol{\eta}):=\sum_{i} \boldsymbol{P}\left\{\boldsymbol{\eta}=\eta_{i}\right\} \eta_{i}=\sum_{i} P_{i} \eta_{i}=\mu
$$

where $\mu$ is called expected, or mean value of $\boldsymbol{\eta}$.

The measure of how far this set of possible values of $\boldsymbol{\eta}$ spread out is called the "variance". For example, if the variance is small, that means the data points tend to be closed to the mean value. By definition, the variance of a random variable $\boldsymbol{\eta}$ is its second central moment, or the expected value of the squared deviation from the mean:

$$
\operatorname{var}(\boldsymbol{\eta}):=E\left[(\boldsymbol{\eta}-\mu)^{2}\right]=E\left(\boldsymbol{\eta}^{2}\right)-(E(\boldsymbol{\eta}))^{2}
$$

The square root of the variance is denoted by $\sigma$, also called the "standard deviation". Fig. 2 shows that about $68 \%$ of the values of a random variable with normal distribution fall within one standard deviation from the mean value; about 95.5\% fall within two standard deviations; and about $99.7 \%$ of the values are within three standard deviation. This fact is well known as the 3-sigma rule, or empirical rule, and will be used to estimate the variability of the outputs of all examples in this work.

For the probabilistic framework of this thesis, we assume that $X(\xi)$ is a stochastic process that represents the uncertain response of the system due to some random variable $\xi$ during the manufacturing process. Since $\xi$ is a random variable, $X(\xi)$ is also a random variable. 


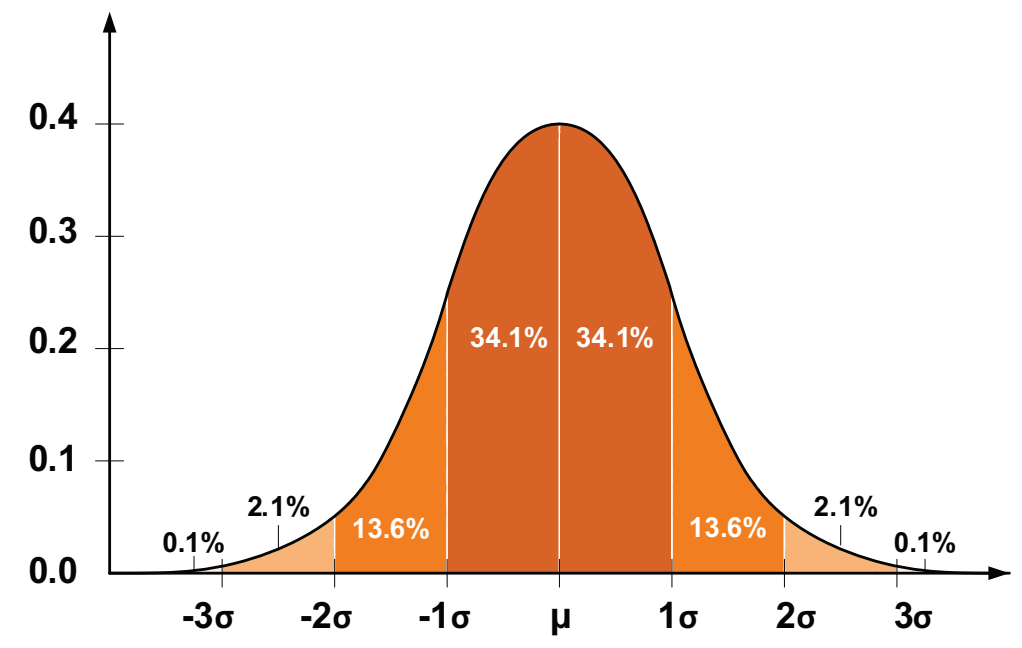

Figure 1: Standard deviation diagram

\subsection{The Monte-Carlo Method}

This section presents the principles of the Monte-Carlo (MC) method as well as some relevant knowledge which will be used in this thesis for the validation purpose.

\subsubsection{Basic knowledge of MC method}

Basically, the MC method is a statistical evaluation tool that relies on repeatedly performing circuit simulations using values assigned to those random parameters, i.e. those circuit parameters that are subject to design uncertainty. The values assigned to those parameters are drawn at random using some random number generator and according to some probability distribution function, e.g. the standard normal distribution. Due to its simplicity in implementation, the MC has become the mainstream statistical analysis tool in almost all CAD suits of circuits and systems. Due to this fact, the MC approach will be used as a reference against which the proposed approach presented in this thesis will evaluate.

Generally, the MC simulation is performed through a process as follows: 
- Define the domain of the random-variable-dependent parameters and their distribution.

- Generate sets of random samples of these parameters over the domain.

- Repeatedly solve the circuit for each deterministic sample generated in the previous step.

- Obtain the variability analysis (the mean and the 3-sigma tolerances) using the results from step 3 .

One of the major difficulties with the MC method is how to generate truly sufficient and efficient random samples [9]. However, many of the most useful schemes of this method in fact do not require these truly random numbers, but use computational algorithms to generate sequences of deterministic pseudo-random results for easy testing and running simulations. Additionally, when dealing with extremely large populations, such as a continuous distribution, it is impossible to count every object in the population, so the variability analysis must be performed on a sample of that population. For the purpose of this work, these samples are generated using MATLAB or HSPICE, with Gaussian distribution, assuming that they are sufficiently "random" enough for statistical analysis. Moreover, these samples will also be used for the proposed algorithm, hence, the number of samples and how to choose them do not affect the comparison between these methods.

The following sections will describe more about the sample mean and standard deviation estimators [10] for use in our stochastic analysis. Generally, an estimator is considered "good" if it is unbiased, which means the expected value of the estimator and the true value of the quantity being estimated are equal. 


\subsubsection{Sample Mean Estimator}

Normally, $\mu$ is a notation of the population mean, which is the mean of a numerical set including all possible numbers within the entire group. For a discrete set of numbers obtained by sampling from some probability distribution, as in this thesis, the term "sample mean" is used as a good estimator of the population mean, since it is unbiased and has the same expected value with the population mean. For the sake of simplifying the mathematical notation, here $\mu$ is used to imply the sample mean.

Particularly, assuming that a Monte-Carlo run contains N simulations, it will provide $\mathrm{N}$ corresponding samples of some stochastic process $\mathrm{X}$ given by $\left\{X=X_{i}\right\}_{i=1}^{N}$, hence, the expected value of $\mathrm{X}$ could be approximated using the discrete form as follows:

$$
\mu=\frac{1}{N} \sum_{i=1}^{N} X_{i}
$$

\subsubsection{Sample Standard Deviation Estimator}

Similar to Sec. 2.2.2, the sample standard deviation will be denoted as $\sigma$ for simplicity. With the same number of samples given above, the approximated variance could be calculated using the biased sample variance:

$$
\operatorname{var}(X)=\sigma^{2}=\frac{1}{N} \sum_{i=1}^{N}\left(X_{i}-\mu\right)^{2}
$$

where $\mu$ is the sample mean as being described in Sec. 2.2.2.

It is easy to find that, since $X_{i}$ is selected randomly, $\sigma^{2}$ is also a random variable itself, hence, its expected value can be evaluated as follows:

$$
E\left(\sigma^{2}\right)=E\left[\frac{1}{N} \sum_{i=1}^{N}\left(X_{i}-\frac{1}{N} \sum_{j=1}^{N} X_{j}\right)^{2}\right]=\frac{N-1}{N} \sigma_{p}^{2}
$$


where $\sigma_{p}$ is the population variance. Since (5) gives an estimation of the population variance that is biased by a factor of $(\mathrm{N}-1) / \mathrm{N}$, the unbiased sample variance is obtained by correcting this difference:

$$
\operatorname{var}(X)=\sigma^{2}=\frac{1}{N-1} \sum_{i=1}^{N}\left(X_{i}-\mu\right)^{2}
$$

Taking the square root of this sample variance yields the "corrected" sample standard deviation, however, this also reintroduces the bias and makes it a biased estimator of the population standard deviation. Although this estimator is biased, it is still remarkably less biased than the uncorrected one obtained from the biased variance. Therefore, this estimator is commonly used, especially for large samples, and generally known as the "sample standard deviation", which will be employed in this thesis.

\subsection{Polynomial Chaos Paradigm}

The previous section described the brute-force Monte-Carlo method for variability analysis, which can turn out to be very computationally expensive and inefficient for many practical applications, especially in complex circuit simulation. To overcome this limitation, another approach has been proposed and developed based on the polynomial chaos theory.

Polynomial chaos is a powerful uncertainty quantification technique which was first proposed in the form of Homogeneous Chaos expansion by N. Wiener [11] in 1938 and its theoretical basis is based on Cameron and Martins theorem [12]. Basically, the idea of the PC approach is to expand a stochastic process into a series of the so-called Askey-Wiener type of orthogonal polynomials [1]. The Hermite orthogonal polynomials were the first such series considered and the optimum basis for Gaussian process since the weighting function of the Hermite polynomials corresponds 
with the probability density function (PDF) of the Gaussian distribution. For other distributions, the convergence rate of the Hermite expansion may be substantially slower, therefore, we could choose another type of polynomials in the Askey scheme which has the weighting function identical to the probability distribution of the random variable. The most popular distributions are shown in Table 1 along with their corresponding optimal class of orthogonal polynomials, which were used to extend the results of the Cameron-Martine principles into a broader framework, popularly known as the generalized polynomial chaos (gPC).

Table 1: Wiener-Askey polynomial chaos and their corresponding optimal random variables

\begin{tabular}{c|c}
\hline Random variables & Wiener-Askey Polynomial Chaos \\
\hline \hline Gaussian & Hermite-Chaos \\
\hline Uniform & Legendre-Chaos \\
\hline Gamma & Laguerre-Chaos \\
\hline Beta & Jacobi-Chaos \\
\hline
\end{tabular}

In the framework of this thesis, all the random variables will be assumed to follow the Gaussian distribution for the purpose of the proposed approach, therefore, we only consider the Hermite Polynomial Chaos. It should be also noted that all these random variables will be assumed to be independent, have zero mean and unit variance.

\subsubsection{Hermite Polynomial Chaos for Single Random Variable}

Consider $\xi$ as a single random variable, $H_{i}(\xi)$ is denoted as the Hermite polynomial of degree " $i$ " where $i=0,1, \cdots$. Basically, the Hermite polynomial can be standardized using the Rodriguez formula by two ways, known as "Probabilists' Hermite polynomials" and "Physicists' Hermite polynomials" [13]. Particularly, the "Probabilists" polynomials are orthogonal with respect to the weighting function $w(\xi)=e^{-\xi^{2} / 2}$ and defined as follows: 


$$
H_{i}(\xi):=(-1)^{i} e^{\xi^{2} / 2} \frac{d^{i}}{d \xi^{i}}\left(e^{-\xi^{2} / 2}\right)
$$

whereas the "Physicists" polynomials are orthogonal with respect to the weighting function $w(\xi)=e^{-\xi^{2}}$ and defined by:

$$
H_{i}(\xi):=(-1)^{i} e^{\xi^{2}} \frac{d^{i}}{d \xi^{i}}\left(e^{-\xi^{2}}\right)
$$

Actually, these two definitions are just the re-scaling versions of each other. However, in the content of this thesis, we only use the "Probabilists" since $\frac{1}{\sqrt{2 \pi}} e^{-\xi^{2} / 2}$ is well-known as the PDF of the Gaussian distribution with zero mean and unit variance. Therefore, "Hermite polynomials" will only be refered to as "Probabilists' Hermite polynomials" in the latter pages, without the term "Probabilists".

In addition to the Rodriguez formula, an alternative way to obtain the Hermite polynomials is to use the three-term recurrence relation [13]:

$$
H_{i+1}(\xi)=\xi H_{i}(\xi)-i H_{i-1}(\xi)
$$

where $i>1$ and given that, $H_{0}=1$ and $H_{1}=\xi$, which could be easily derived from $(7)$.

Similar to other orthogonal polynomials, the system of Hermite polynomials also satisfies the orthogonality relation as follows:

$$
\int_{-\infty}^{\infty} H_{i}(\xi) H_{j}(\xi) w(\xi) d \xi=\int_{-\infty}^{\infty} e^{-\xi^{2} / 2} H_{i}(\xi) H_{j}(\xi) d \xi=\sqrt{2 \pi} i ! \delta_{i j}
$$

where $\delta_{i j}$ is the Kronecker delta function $\left(\delta_{i j}=1\right.$ when $i=j$ and $\delta_{i j}=0$ when $i \neq j$ ). It should also be noted that the equation (10) is equivalent to an inner product $<H_{i}(\xi), H_{j}(\xi)>$.

Now consider $\mathrm{X}$ as a stochastic process of the single random variable $\xi$, then $\mathrm{X}$ 
can be expanded in terms of the Hermite polynomial series as follows:

$$
X(\xi)=\sum_{i=0}^{\infty} X_{i} H_{i}(\xi)
$$

where $X_{i}$ is a scalar expansion coefficient corresponding to the Hermite polynomial $H_{i}(\xi)$. In practical applications, the Hermite expansion above is truncated at a certain order $\mathrm{M}$, which generates $M+1$ terms of Hermite polynomials. For example, if $\mathrm{M}=4$, the Hermite polynomials of order 0 to 4 can be easily derived from (9), as shown in Table 2.

Table 2: Hermite polynomials of orders up to $M=4$

\begin{tabular}{c|l}
\hline Order $(i)$ & Hermite Polynomial $\left(H_{i}\right)$ \\
\hline \hline 0 & $H_{0}=1$ \\
\hline 1 & $H_{1}=\xi$ \\
\hline 2 & $H_{2}=\xi^{2}-1$ \\
\hline 3 & $H_{3}=\xi^{3}-3 \xi$ \\
\hline 4 & $H_{4}=\xi^{4}-6 \xi^{2}+3$ \\
\hline
\end{tabular}

Once the coefficients $X_{i}$ are made available, then computing the statistical properties of the stochastic process $X(\xi)$, e.g. mean $\mu$ and variance $\sigma^{2}$, can be obtained analytically through these coefficients. For example, the mean $\mu$ is given by

$$
\mu=X_{0}
$$

while the standard deviation, $\sigma$, is given by

$$
\sigma^{2}=\sum_{i=1}^{M} X_{i}^{2} i !
$$




\subsubsection{Hermite Polynomial Chaos for Multi-random Variables}

For the case of multi-dimensional random space $\Omega$, first let $\boldsymbol{\xi}=\left[\xi_{1}, \cdots, \xi_{d}\right]$ be a set of $d$ random variables which maps $\Omega$, into $\mathbb{R}^{d}$, and $\phi_{\boldsymbol{\alpha}}(\boldsymbol{\xi})$ be the multi-dimensional polynomials that are orthogonal with respect to a probability measure $W(\boldsymbol{\xi})$ with support on $\Omega$. A multi-index, e.g. $\boldsymbol{\alpha} \in \mathbb{N}^{d}$, is also defined as a vector whose entries are non-negative integers. In addition, an index, written in a non-bold font, with a subscript, e.g. $\alpha_{i}$, is used to refer to the $i^{\text {th }}$ component of a multi-index $\boldsymbol{\alpha}$.

The system of multivariate Hermite polynomials satisfies the orthogonality relation as follows:

$$
<\phi_{\boldsymbol{\alpha}}(\boldsymbol{\xi}), \phi_{\boldsymbol{\beta}}(\boldsymbol{\xi})>=\int_{\Omega} \phi_{\boldsymbol{\alpha}}(\boldsymbol{\xi}) \phi_{\beta}(\boldsymbol{\xi}) W(\boldsymbol{\xi}) d \boldsymbol{\xi}=<\phi_{\boldsymbol{\alpha}}(\boldsymbol{\xi})^{2}>\delta_{\boldsymbol{\alpha} \boldsymbol{\beta}}
$$

where $\delta_{\boldsymbol{\alpha} \boldsymbol{\beta}}$ is the Kronecker delta function as described in the previous section and

$W(\boldsymbol{\xi})=\frac{1}{\sqrt{2 \pi^{d}}} e^{-\|\boldsymbol{\xi}\|^{2} / 2}$ which is identical to the $d$-dimensional Gaussian probability density function [1].

Now we assume that $X(\boldsymbol{\xi})$ is a stochastic process that represents the uncertain response of the system due to multi-random variables in $\boldsymbol{\xi}$. The PC-based variability analysis is based on expanding $X(\boldsymbol{\xi})$ as a series of the form

$$
X(\boldsymbol{\xi})=\sum_{\boldsymbol{\alpha}} a_{\boldsymbol{\alpha}} \phi_{\boldsymbol{\alpha}}(\boldsymbol{\xi})
$$

where $a_{\boldsymbol{\alpha}}$ is a set of scalar coefficients, and $\phi_{\boldsymbol{\alpha}}(\boldsymbol{\xi})$ are multi-dimensional polynomials that are orthogonal with respect to a probability measure $w(\boldsymbol{\xi})$ with support on $\Omega$. The subscript $\boldsymbol{\alpha}$ may be thought of as a unique vector-valued label attached to each term in the series (15).

Truncating the series in (15) and ordering its terms are two principal ideas in multi-dimensional polynomials, that will be discussed next. 


\subsubsection{Truncation Schemes}

Truncation refers to specifying criteria on multi-indices whose corresponding coefficients are to be included in the series. In fact, there are several approaches to specify those criteria. For example, one of the common approaches is to use the concept of "total order" which includes only those terms whose multi-indices coefficients satisfy $\sum_{i=1}^{d} \alpha_{i} \leq M$, for a given positive integer $M$. This truncation scheme leads to the total number $N_{\text {total }}$ of coefficients as follows:

$$
N_{\text {total }}=\frac{(d+M) !}{d ! M !}
$$

An example of ordering the multi-indices for the case of $d=2$ and $M=2$, where $N_{\text {total }}$ turns out to be equal to 6 , is presented in Table 3. The general expression of the multivariate Hermite polynomials can be obtained by simply applying the three term recurrence relation in (9) to the multi-random variable case.

Table 3: An example of ordering $\boldsymbol{\alpha}$ using total order where $d=2$ and $M=2$

\begin{tabular}{c|c|l}
\hline Total order & Multi-index $(\boldsymbol{\alpha})$ & Hermite Polynomial $\phi_{\boldsymbol{\alpha}}(\boldsymbol{\xi})=\phi_{\alpha_{i}}\left(\xi_{1}\right) \phi_{\alpha_{j}}\left(\xi_{2}\right)$ \\
\hline \hline 0 & {$[0,0]$} & $\phi_{00}(\boldsymbol{\xi})=\phi_{0}\left(\xi_{1}\right) \phi_{0}\left(\xi_{2}\right)=1$ \\
\hline \hline 1 & {$[1,0]$} & $\phi_{10}(\boldsymbol{\xi})=\phi_{1}\left(\xi_{1}\right) \phi_{0}\left(\xi_{2}\right)=\xi_{1}$ \\
\hline \hline 1 & {$[0,1]$} & $\phi_{01}(\boldsymbol{\xi})=\phi_{0}\left(\xi_{1}\right) \phi_{1}\left(\xi_{2}\right)=\xi_{2}$ \\
\hline 2 & {$[2,0]$} & $\phi_{20}(\boldsymbol{\xi})=\phi_{2}\left(\xi_{1}\right) \phi_{0}\left(\xi_{2}\right)=\xi_{1}^{2}-1$ \\
\hline 2 & {$[0,2]$} & $\phi_{02}(\boldsymbol{\xi})=\phi_{0}\left(\xi_{1}\right) \phi_{2}\left(\xi_{2}\right)=\xi_{2}^{2}-1$ \\
\hline 2 & {$[1,1]$} & $\phi_{11}(\boldsymbol{\xi})=\phi_{1}\left(\xi_{1}\right) \phi_{1}\left(\xi_{2}\right)=\xi_{1} \xi_{2}$ \\
\hline
\end{tabular}

The total order allows handling the special case of isotropic random space, i.e., those spaces that show identical properties with respect to all the random variables. However, handling the general case of anisotropic random spaces, requires using the "tensor order" [14]. Translating this to our problems, the "tensor order" allows us to choose different expansion orders for different random variables. The flexibility 
of "tensor order" is practically important due to the fact that the variations in the random variables are normally not equal. Particularly, the "tensor order" allows terms in the truncated series only if their multi-indices satisfy $\alpha_{i} \leq p_{i}, \forall i$ for a given positive integer, $p_{i}$. Hence, the total number $N_{\text {tensor }}$ of coefficients is given by:

$$
N_{\text {tensor }}=\prod_{i=1}^{d}\left(p_{i}+1\right)
$$

In addition to its generality, the "tensor order" also lends itself naturally to the proposed decoupling formulation presented in the thesis. Therefore, we adopt the "tensor order" as the truncation scheme throughout this work. For the sake of simplifying the mathematical notation in our derivation in latter chapters, we assume $p_{i}=M$, for $i=1, \cdots, d$. Hence, the total number of coefficients in the case of tensor order $N_{\text {tensor }}$ is given as the following:

$$
N_{\text {tensor }}=(M+1)^{d}
$$

For the same example of total order above, where $d=2$ and $M=2, N_{\text {tensor }}=9$.

\subsubsection{Ordering the Multi-indices}

The choice of ordering the terms in the truncated series also impacts the presentation of the theoretical results. In fact, one of the essential differences between polynomials in a single variable and polynomials in several variables, such as $\phi_{\boldsymbol{\alpha}}(\boldsymbol{\xi})$, is the lack of a natural order in the latter. Instead, there are many ways to order the polynomials $\phi_{\boldsymbol{\alpha}}(\boldsymbol{\xi})$ and their coefficients $a_{\boldsymbol{\alpha}}$ according to the contents of the multi-index.

For the tensor order which is used for the proposed approach, we define the ordering by assuming, without loss of generality, that $0 \leq \alpha_{i} \leq M$ for $i=1, \cdots, d$ and 
denote the rank of a multi-index $\boldsymbol{\alpha}$ by $|\boldsymbol{\alpha}|$, where,

$$
|\boldsymbol{\alpha}|:=\sum_{i=1}^{d} \alpha_{i}(M+1)^{(d-i)}
$$

Table 4 illustrates the ordering of multi-indices for the case of $d=2$, and $M=2$ according to the ranking defined by (19), where $N_{\text {tensor }}$ turns out to be equal to 9 .

Table 4: An example of ordering $\boldsymbol{\alpha}$ using tensor order where $d=2$ and $M=2$

\begin{tabular}{c|c|c|c}
\hline$\alpha_{1}$ & $\alpha_{2}$ & $\phi_{\boldsymbol{\alpha}}$ & $|\boldsymbol{\alpha}|$ \\
\hline \hline 0 & 0 & $\phi_{00}$ & 0 \\
\hline 0 & 1 & $\phi_{01}$ & 1 \\
\hline 0 & 2 & $\phi_{02}$ & 2 \\
\hline 1 & 0 & $\phi_{10}$ & 3 \\
\hline 1 & 1 & $\phi_{11}$ & 4 \\
\hline 1 & 2 & $\phi_{12}$ & 5 \\
\hline 2 & 0 & $\phi_{20}$ & 6 \\
\hline 2 & 1 & $\phi_{21}$ & 7 \\
\hline 2 & 2 & $\phi_{22}$ & 8 \\
\hline
\end{tabular}

For the total order, we assume that the multi-indices are ordered according to the Lexicographic order [15], which is defined as, "a multi-index $\boldsymbol{\alpha}$ is said to be of higher order than another multi-index, say $\boldsymbol{\beta}$, and denoted by $\boldsymbol{\alpha} \succ \boldsymbol{\beta}$, if the first nonzero entry in the difference is positive". The advantage of the total lexicographic order is that, it provides the same order of rank as the tensor order, which allows us to construct the augmented circuit system of DAEs with the total order from the one with tensor order, described in later chapter. Moreover, the multi-indices of the total lexicographic order where $\sum_{i=1}^{d} \alpha_{i} \leq M$ is also a sub-set of the multi-index set in case of tensor order where $\alpha_{i} \leq M$, for $i=1, \cdots, d$. This means, for a set of multi-indices $\boldsymbol{\alpha}$, the all-zero, 0, multi-index is the first element, with the lowest order, while the last element associated with the highest order will be $[M, 0, \cdots, 0]$. 
Particularly, for the example of tensor order where $d=2$, and $M=2$ in Section. 1 , if we use the total order, where $\sum_{i=1}^{d} \alpha_{i} \leq M=2$, and sort it according to lexicographic scheme, the order of multi-indices will be presented as in Table. 5.

Table 5: An example of ordering $\boldsymbol{\alpha}$ using total lexicographic order where $d=2$ and $M=2$

\begin{tabular}{c|c|c|c}
\hline$\alpha_{1}$ & $\alpha_{2}$ & $\phi_{\boldsymbol{\alpha}}$ & $|\boldsymbol{\alpha}|$ \\
\hline \hline 0 & 0 & $\phi_{00}$ & 0 \\
\hline 0 & 1 & $\phi_{01}$ & 1 \\
\hline 0 & 2 & $\phi_{02}$ & 2 \\
\hline 1 & 0 & $\phi_{10}$ & 3 \\
\hline 1 & 1 & $\phi_{11}$ & 4 \\
\hline 2 & 0 & $\phi_{20}$ & 6 \\
\hline
\end{tabular}

Using the tensor order, from the orthogonality relation in (14), the inner product of any two polynomials, $\phi_{\boldsymbol{\alpha}}$ and $\phi_{\boldsymbol{\beta}}$ is equivalent to the following expression:

$$
<\phi_{\boldsymbol{\alpha}}(\boldsymbol{\xi}), \phi_{\boldsymbol{\beta}}(\boldsymbol{\xi})>=\int_{\Omega} \phi_{\boldsymbol{\alpha}}(\boldsymbol{\xi}) \phi_{\boldsymbol{\beta}}(\boldsymbol{\xi}) W(\boldsymbol{\xi}) \mathrm{d} \boldsymbol{\xi}=\prod_{i=1}^{d} \kappa_{i} \delta_{\alpha_{i} \beta_{i}}
$$

where $\kappa_{i}$ is a positive constant and $\delta_{\alpha_{i} \beta_{i}}$ is the Kronecker delta function, which is equal to 1 if, and only if, $\alpha_{i}=\beta_{i}$; otherwise it is identically 0 . It should be noted again that $W(\boldsymbol{\xi})=\frac{1}{\sqrt{2 \pi^{d}}} e^{-\|\boldsymbol{\xi}\|^{2} / 2}$ is the probability measure with support on $\Omega$.

As mentioned in Sec. 2.3.1, the PC-based variability analysis enables representing the statistical properties of the stochastic process $X(\boldsymbol{\xi})$ analytically through these coefficients of this expansion. Particularly, the mean and the standard deviation are given by $\mu=a_{\mathbf{0}}$ and $\sigma^{2}=\sum_{\boldsymbol{\alpha}, \boldsymbol{\alpha} \neq \mathbf{0}} a_{\boldsymbol{\alpha}}^{2}$, respectively. However, for frequency analysis with complex values, estimation of the statistical properties of the magnitude, or phase, of the stochastic process $X(\boldsymbol{\xi})$ requires using the sample mean and standard deviation estimators. Moreover, validating the precision of the proposed PC-based algorithm using this approach provides a fair comparison to the MC-based method, 
which also uses the sample estimators, no matter how many MC runs are taken. Therefore, it will be adopted in all the numerical validation presented in latter chapters of this thesis. 


\section{Chapter 3}

\section{Applications of Polynomial-Chaos to Circuit Variability Analysis}

This chapter considers the application of the PC to quantify the variability of circuit performance. Particularly, Section 3.1 will describe how to construct the augmented system of circuit equations arising from the Hermite PC-based analysis in the frequency domain, which is based on modifications of the well-known modified nodal analysis (MNA) [16] and the Galerkin projection process [17]. An illustrated example of this augmented system will be provided later in Section 3.2 before we outline the computational limitations of the recent PC-based approaches in Section 3.3.

\subsection{Frequency Domain Variability Analysis}

In the context of circuit or EM simulation, random variables are those physical or electrical components whose values are subject to significant uncertainty, while the stochastic processes are the node voltages, branch currents, and capacitor and inductor charges and fluxes. This notion is captured by using the MNA to represent the circuit as follows,

$$
(\boldsymbol{G}+s \boldsymbol{C}) \boldsymbol{X}(s)=\boldsymbol{U}
$$


where $\boldsymbol{G}, \boldsymbol{C} \in \mathbb{R}^{N \times N}$ are matrices describing the memoryless and memory elements in the circuit, respectively; $\boldsymbol{X}(s) \in \mathbb{C}^{N}$ is a vector of the voltages of circuit nodes, currents in the inductors or independent voltage sources; and $\boldsymbol{U} \in \mathbb{C}^{N}$ is a vector representing the independent stimulus of the circuit, with $N$ representing the number of the variables in the circuit response and $s=j \omega$.

In order to capture the idea of uncertainty in the circuit response due to uncertainty in the parameters of the fabrication process, the MNA formulation in (21) is modified as follows

$$
(\boldsymbol{G}(\boldsymbol{\xi})+s \boldsymbol{C}(\boldsymbol{\xi})) \boldsymbol{X}(s, \boldsymbol{\xi})=\boldsymbol{U}
$$

where $\boldsymbol{\xi} \in \mathbb{R}^{d}$ is a vector of $d$ random variables that represents the randomness or the uncertainty of the manufacturing process.

The starting point of the Hermite-based PC variability analysis is the adoption of the generalized Hermite polynomials, denoted by $\mathcal{H}_{\boldsymbol{\alpha}}(\boldsymbol{\xi})$, for the generic orthogonal polynomials $\phi_{\boldsymbol{\alpha}}(\boldsymbol{\xi})$. Typically, $\mathcal{H}_{\boldsymbol{\alpha}}(\boldsymbol{\xi})$ are given by

$$
\mathcal{H}_{\boldsymbol{\alpha}}(\boldsymbol{\xi})=\prod_{i=1}^{d} H_{\alpha_{i}}\left(\xi_{i}\right)
$$

where $\alpha_{i}$ are the non-negative integer elements of the multi-index $\boldsymbol{\alpha}$, and $H_{m}\left(\xi_{i}\right),(m$ being a non-negative integer) is the single-dimensional $m^{\text {th }}$ degree Hermite polynomial [18]. 
Next, all the $\boldsymbol{\xi}$-dependent terms in (22) are expanded as a series of Hermite polynomials in $\boldsymbol{\xi}$. Thus we have,

$$
\begin{aligned}
\boldsymbol{G}(\boldsymbol{\xi}) & =\sum_{\boldsymbol{\beta}} \hat{\boldsymbol{G}}_{\boldsymbol{\beta}} \mathcal{H}_{\boldsymbol{\beta}}(\boldsymbol{\xi}) \\
\boldsymbol{C}(\boldsymbol{\xi}) & =\sum_{\boldsymbol{\beta}} \hat{\boldsymbol{C}}_{\boldsymbol{\beta}} \mathcal{H}_{\boldsymbol{\beta}}(\boldsymbol{\xi}) \\
\boldsymbol{X}(s, \boldsymbol{\xi}) & =\sum_{\boldsymbol{\alpha}} \boldsymbol{X}_{\boldsymbol{\alpha}}(s) \mathcal{H}_{\boldsymbol{\alpha}}(\boldsymbol{\xi})
\end{aligned}
$$

where $\boldsymbol{\alpha}$ and $\boldsymbol{\beta}$ are the $d$-dimensional multi-indices,

$$
\begin{aligned}
& \boldsymbol{\alpha}=\left[\begin{array}{llll}
\alpha_{1} & \alpha_{2} & \cdots & \alpha_{d}
\end{array}\right] \\
& \boldsymbol{\beta}=\left[\begin{array}{llll}
\beta_{1} & \beta_{2} & \cdots & \beta_{d}
\end{array}\right]
\end{aligned}
$$

In order to enable the following derivations to be general, we let the elements of the multi-index $\boldsymbol{\beta}$ be truncated at an upper bound integer $Q$, while those of the $\boldsymbol{\alpha}$ are truncated at $M$, as before. Thus, $0 \leq \alpha_{i} \leq M$ and $0 \leq \beta_{i} \leq Q$ for $i=1, \cdots, d$, and we assume that $M \geq Q$. The order of the multi-indices is taken according to the ranking system of the tensor order described in (19). For example, when $d=2, \boldsymbol{G}(\boldsymbol{\xi})$ could be expressed as:

$$
\begin{aligned}
\boldsymbol{G}(\boldsymbol{\xi}) \cong \sum_{\boldsymbol{\beta}} \hat{\boldsymbol{G}}_{\boldsymbol{\beta}} \mathcal{H}_{\boldsymbol{\beta}}(\boldsymbol{\xi}) & \\
= & \hat{\boldsymbol{G}}_{00} \mathcal{H}_{00}(\boldsymbol{\xi})+\hat{\boldsymbol{G}}_{01} \mathcal{H}_{01}(\boldsymbol{\xi})+\cdots+\hat{\boldsymbol{G}}_{0 Q} \mathcal{H}_{0 Q}(\boldsymbol{\xi}) \\
& +\hat{\boldsymbol{G}}_{10} \mathcal{H}_{10}(\boldsymbol{\xi})+\hat{\boldsymbol{G}}_{11} \mathcal{H}_{11}(\boldsymbol{\xi})+\cdots+\hat{\boldsymbol{G}}_{1 Q} \mathcal{H}_{0 Q}(\boldsymbol{\xi}) \\
\vdots & \\
& +\hat{\boldsymbol{G}}_{Q 0} \mathcal{H}_{Q 0}(\boldsymbol{\xi})+\hat{\boldsymbol{G}}_{Q 1} \mathcal{H}_{Q 1}(\boldsymbol{\xi})+\cdots+\hat{\boldsymbol{G}}_{Q Q} \mathcal{H}_{Q Q}(\boldsymbol{\xi})
\end{aligned}
$$


The ultimate goal of using the PC-based approach to variability analysis is to compute the coefficients $\boldsymbol{X}_{\boldsymbol{\alpha}}(s)$ and use them to deduce the statistical properties of $\boldsymbol{X}(s, \boldsymbol{\xi})$. To this end, a Galerkin projection procedure is carried out through the following steps,

- substitution from (24)-(26) into (22),

- multiplication by $\mathcal{H}_{\boldsymbol{\gamma}}(\boldsymbol{\xi}) e^{-\|\boldsymbol{\xi}\|^{2} / 2}$ where $\boldsymbol{\gamma}$ is an arbitrary multi-index given by

$$
\gamma=\left[\begin{array}{llll}
\gamma_{1} & \gamma_{2} & \cdots & \gamma_{d}
\end{array}\right]
$$

- integration over the $d$-dimensional cube from $-\infty$ to $\infty$ in each dimension, and

- substitution, using the closed-form of the multi-dimensional triple product integral, given by

$$
\Theta_{\boldsymbol{\alpha}, \boldsymbol{\beta}, \boldsymbol{\gamma}}:=\int_{-\infty}^{\infty} \cdots \int_{-\infty}^{\infty} \mathcal{H}_{\boldsymbol{\alpha}}(\boldsymbol{\xi}) \mathcal{H}_{\boldsymbol{\beta}}(\boldsymbol{\xi}) \mathcal{H}_{\boldsymbol{\gamma}}(\boldsymbol{\xi}) e^{-\|\boldsymbol{\xi}\|^{2} / 2} \mathrm{~d} \xi_{1} \cdots \mathrm{d} \xi_{d}=\prod_{i=1}^{d} \theta_{\alpha_{i}, \beta_{i}, \gamma_{i}}
$$

where $\theta_{\alpha_{i}, \beta_{i}, \gamma_{i}}$ is the integral of one-dimensional triple product given by

$$
\begin{aligned}
& \theta_{m, n, p}:=\int_{-\infty}^{\infty} H_{m}\left(\xi_{i}\right) H_{n}\left(\xi_{i}\right) H_{p}\left(\xi_{i}\right) e^{-\xi_{i}^{2} / 2} \mathrm{~d} \xi_{i}
\end{aligned}
$$

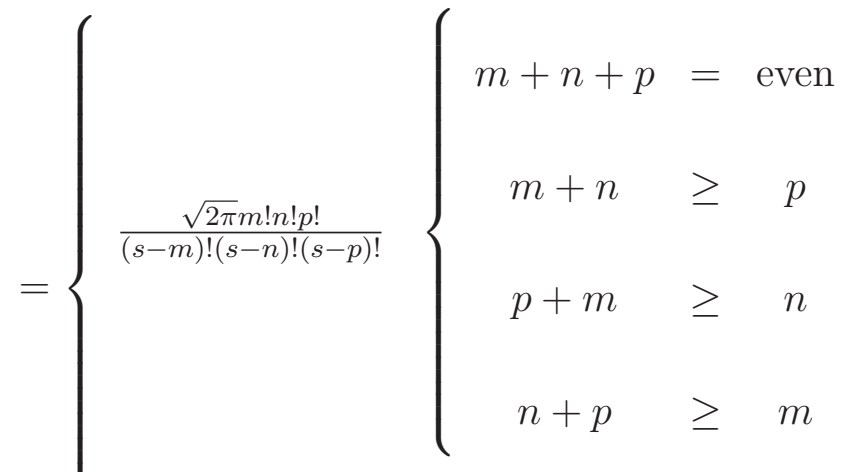

$$
\begin{aligned}
& 0 \quad \text { otherwise }
\end{aligned}
$$


with $s=\frac{m+n+p}{2}$.

The above process typically results in the following system of equations:

$$
\begin{aligned}
\sum_{\boldsymbol{\alpha}}\left(\sum_{\boldsymbol{\beta}} \hat{\boldsymbol{G}}_{\boldsymbol{\beta}} \Theta_{\boldsymbol{\alpha}, \boldsymbol{\beta}, \boldsymbol{\gamma}}+s \sum_{\boldsymbol{\beta}} \hat{\boldsymbol{C}}_{\boldsymbol{\beta}} \Theta_{\boldsymbol{\alpha}, \boldsymbol{\beta}, \boldsymbol{\gamma}}\right) & \boldsymbol{X}_{\boldsymbol{\alpha}}(s)= \\
& \left\{\begin{array}{cc}
(2 \pi)^{d / 2} \boldsymbol{U} & \boldsymbol{\gamma}=\left[\begin{array}{cccc}
0 & 0 & \cdots & 0
\end{array}\right] \\
0 & \text { otherwise }
\end{array}\right.
\end{aligned}
$$

To simplify the derivation, let us define:

$$
\overline{\boldsymbol{\Theta}}_{\boldsymbol{\alpha}, \boldsymbol{\beta}, \boldsymbol{\gamma}}:=\frac{\boldsymbol{\Theta}_{\boldsymbol{\alpha}, \boldsymbol{\beta}, \boldsymbol{\gamma}}}{(2 \pi)^{d / 2} \prod_{i=1}^{d} \gamma_{i} !}
$$

Using the normalized $\overline{\boldsymbol{\Theta}}_{\boldsymbol{\alpha}, \boldsymbol{\beta}, \boldsymbol{\gamma}}$ instead of $\boldsymbol{\Theta}_{\boldsymbol{\alpha}, \boldsymbol{\beta}, \boldsymbol{\gamma}}$ in (33) leads to rewriting this system in the following form:

$$
\begin{aligned}
\sum_{\alpha}\left(\sum_{\boldsymbol{\beta}} \hat{\boldsymbol{G}}_{\boldsymbol{\beta}} \bar{\Theta}_{\boldsymbol{\alpha}, \boldsymbol{\beta}, \boldsymbol{\gamma}}+s \sum_{\boldsymbol{\beta}} \hat{\boldsymbol{C}}_{\boldsymbol{\beta}} \bar{\Theta}_{\boldsymbol{\alpha}, \boldsymbol{\beta}, \boldsymbol{\gamma}}\right) \boldsymbol{X}_{\boldsymbol{\alpha}}(s)= & \left\{\begin{array}{cc}
\boldsymbol{U} & \boldsymbol{\gamma}=\left[\begin{array}{cccc}
0 & 0 & \cdots & 0
\end{array}\right] \\
0 & \text { otherwise }
\end{array}\right.
\end{aligned}
$$

It should be noted that carrying out the above process for an arbitrary multi-index $\gamma$ has produced a system with only $N$ equations while involving $(M+1)^{d} N$ unknown coefficients $\boldsymbol{X}_{\boldsymbol{\alpha}}$. Thus, to balance the system, this process needs to be repeated by sweeping the multi-index $\gamma$ over the $(M+1)^{d}$ possible values. This generates the following system of equations

$$
(\overline{\boldsymbol{G}}+s \overline{\boldsymbol{C}}) \overline{\boldsymbol{X}}(s)=\overline{\boldsymbol{U}}
$$


where $\overline{\boldsymbol{G}}, \overline{\boldsymbol{C}} \in \mathbb{R}^{N\left(N_{\text {tensor }}\right) \times N\left(N_{\text {tensor }}\right)}$ are augmented matrices, that are constructed from the matrix-valued Hermite coefficients $\hat{\boldsymbol{G}}_{\boldsymbol{\beta}}$ and $\hat{\boldsymbol{C}}_{\boldsymbol{\beta}}$, and $\overline{\boldsymbol{X}}(s) \in \mathbb{R}^{N(M+1)^{d}}$ is given by

$$
\overline{\boldsymbol{X}}(s)=\left[\begin{array}{llll}
\hat{\boldsymbol{X}}_{1}(s)^{\top} & \hat{\boldsymbol{X}}_{2}(s)^{\top} & \cdots & \hat{\boldsymbol{X}}_{(M+1)^{d}}(s)^{\top}
\end{array}\right]^{\top}
$$

with $\hat{\boldsymbol{X}}_{i}(s) \in \mathbb{C}^{N}$ being a vector grouping all Hermite coefficients of those Hermite polynomials whose rank is equal to $i$, for all the $N$ circuit variables of the MNA formulation, and $T$ denotes the transpose operator.

\subsection{Illustrative Example of the Augmented Sys- tem}

To illustrate constructing the augmented system of (36), we use a simple example, which is assumed that $d=1$ and $M=3$. In this case, the $\overline{\boldsymbol{G}}$ and $\overline{\boldsymbol{C}}$ are written as follows [19],

$$
\begin{gathered}
\overline{\boldsymbol{G}}=\left(\begin{array}{cccc}
\hat{\boldsymbol{G}}_{0} & \hat{\boldsymbol{G}}_{1} & \hat{\boldsymbol{G}}_{2} & 6 \hat{\boldsymbol{G}}_{3} \\
\hat{\boldsymbol{G}}_{1} & \hat{\boldsymbol{G}}_{0}+2 \hat{\boldsymbol{G}}_{2} & 2 \hat{\boldsymbol{G}}_{1}+6 \hat{\boldsymbol{G}}_{3} & \hat{\boldsymbol{G}}_{3} \\
\hat{\boldsymbol{G}}_{2} & \hat{\boldsymbol{G}}_{1}+3 \hat{\boldsymbol{G}}_{3} & \hat{\boldsymbol{G}}_{0}+4 \hat{\boldsymbol{G}}_{2} & 18 \hat{\boldsymbol{G}}_{3} \\
\hat{\boldsymbol{G}}_{3} & \hat{\boldsymbol{G}}_{2} & \hat{\boldsymbol{G}}_{1}+6 \hat{\boldsymbol{G}}_{3} & \hat{\boldsymbol{G}}_{0}+6 \hat{\boldsymbol{G}}_{2}
\end{array}\right) \\
\overline{\boldsymbol{C}}=\left(\begin{array}{cccc}
\hat{\boldsymbol{C}}_{0} & \hat{\boldsymbol{C}}_{1} & \hat{\boldsymbol{C}}_{2} & 6 \hat{\boldsymbol{C}}_{3} \\
\hat{\boldsymbol{C}}_{1} & \hat{\boldsymbol{C}}_{0}+2 \hat{\boldsymbol{C}}_{2} & 2 \hat{\boldsymbol{C}}_{1}+6 \hat{\boldsymbol{C}}_{3} & \hat{\boldsymbol{C}}_{3} \\
\hat{\boldsymbol{C}}_{2} & \hat{\boldsymbol{C}}_{1}+3 \hat{\boldsymbol{C}}_{3} & \hat{\boldsymbol{C}}_{0}+4 \hat{\boldsymbol{C}}_{2} & 18 \hat{\boldsymbol{C}}_{3} \\
\hat{\boldsymbol{C}}_{3} & \hat{\boldsymbol{C}}_{2} & \hat{\boldsymbol{C}}_{1}+6 \hat{\boldsymbol{C}}_{3} & \hat{\boldsymbol{C}}_{0}+6 \hat{\boldsymbol{C}}_{2}
\end{array}\right)
\end{gathered}
$$




\subsection{Difficulties with the PC Approach}

As can be seen easily from the above, the size of the augmented system grows rapidly with the increasing numbers of random parameter in the physical design. This phenomena limits the attractiveness of the PC approach in tackling large-scale problems in variability analysis.

The main objective of this work is to address the growth in computational complexity arising from the large size of the augmented matrices. The approach followed to achieve this goal is based on developing the general theory that describes the structure of those matrices. To the best of the author's knowledge, the present literature only describes the structures of the augmented matrices. However, a general theory that provides analytical formulas for their structures is still lacking.

The key advantage of the new theory is, derived in the following sections, it reveals certain characteristics in the augmented matrices that can be used to decouple those matrices, which uses the tensor order, into matrices of size $N$, which can then be factorized independently or in parallel on suitable platforms. In addition, the new analytical formulas also show that it is possible to construct the augmented system of DAEs with total order from the corresponding decoupling augmented system which employs the tensor order. 


\section{Chapter 4}

\section{Closed-form Representation of}

\section{Augmented Matrices arising from Hermite-based PC}

In this chapter, details of the proposed algorithm for obtaining the closed-form representation of the augmented matrices in (36) are given by the proposal of four lemmas

along with their proofs. Throughout the first three lemmas, the presentation of the new theory will be considered, with the case of the PC approach under the assumption of a single random parameter, in Section 4.1. This will be followed by Lemma 4, which is a presentation of the generalized treatment for the case of multiple random parameters, in Section 4.2. The last section of this chapter will present how to construct the augmented matrices with total order from the corresponding augmented system using tensor order.

\subsection{The Case of a Single Random Variable}

In the case where the variability analysis involves only one random parameter, the used notation is considerably simplified. For example, the Hermite expansions in 
(24)-(26) can be represented using a single index as follows

$$
\begin{aligned}
\boldsymbol{G}(\xi) & =\sum_{i=0}^{Q} \hat{\boldsymbol{G}}_{i} H_{i}(\xi) \\
\boldsymbol{C}(\xi) & =\sum_{i=0}^{Q} \hat{\boldsymbol{C}}_{i} H_{i}(\xi) \\
\boldsymbol{X}(s, \xi) & =\sum_{j=0}^{M} \boldsymbol{X}_{j}(s) H_{j}(\xi)
\end{aligned}
$$

\subsubsection{Lemma 1}

The following lemma describes the structure of the augmented matrices $\overline{\boldsymbol{G}}$ and $\overline{\boldsymbol{C}}$ in (36).

Lemma 1. For the case of a single random variable, the augmented matrices $\overline{\boldsymbol{G}}$ and $\overline{\boldsymbol{C}}$ can be described as follows

$$
\begin{aligned}
\overline{\boldsymbol{G}} & =\sum_{i=0}^{Q} \boldsymbol{K}_{i} \otimes \hat{\boldsymbol{G}}_{i} \\
\overline{\boldsymbol{C}} & =\sum_{i=0}^{Q} \boldsymbol{K}_{i} \otimes \hat{\boldsymbol{C}}_{i}
\end{aligned}
$$

where $\otimes$ denotes the Kronecker product operator, [20], and $\boldsymbol{K}_{i} \in \mathbb{R}^{(M+1) \times(M+1)}$ are matrices whose entries are described as follows. Let $K_{i}^{(j, k)}$ denote the entry at the $(k+1)^{\text {th }}$ row and the $(j+1)^{\text {th }}$ column of $\boldsymbol{K}_{i}$, where $0 \leq(k, j) \leq M$. Then $K_{i}^{(j, k)}$ is given as follow, where $p=(j-k+i) / 2$. 


$$
K_{i}^{(j, k)}=\left\{\begin{array}{cl}
\frac{i !(k-i+2 p) !}{p !(i-p) !(k-i+p) !} & \text { for, } j=k-i, k-i+2, \cdots, k+i \\
0 & \text { for, } j \text { otherwise }
\end{array}\right\} k>i
$$

Proof. This lemma can be proved by investigating the coefficients $\boldsymbol{X}_{j}$ that are multiplied by a coefficient matrix $\hat{\boldsymbol{G}}_{i}$ or $\hat{\boldsymbol{C}}_{i}$. To proceed, we rewrite (35) for the single random parameter formulation as follows,

$$
\sum_{i=0}^{Q} \sum_{j=0}^{M} \boldsymbol{G}_{i} \boldsymbol{X}_{j} \bar{\theta}_{i, j, k}+s \sum_{i=0}^{Q} \sum_{j=0}^{M} \boldsymbol{C}_{i} \boldsymbol{X}_{j} \bar{\theta}_{i, j, k}=\left\{\begin{array}{cc}
\boldsymbol{U} & k=0 \\
0 & k>0
\end{array}\right.
$$

where $\bar{\theta}_{i, j, k}=\frac{\theta_{i, j, k}}{\sqrt{2 \pi k !}}$.

It should be stressed that the above system is a system of $N$ equations that involves $(M+1) N$ unknowns that was generated for an arbitrary $k$. To produce a number of equations equal to the number of unknowns, $k$ is changed from 0 to $M$.

The goal of the following analysis is to identify the indices $j$, along with the numerical values of $\bar{\theta}_{i, j, k}$, whose corresponding coefficients $\boldsymbol{X}_{j}$ is paired with $\boldsymbol{G}_{i}$ for a 
given value of the integer $k$. We investigate those for the following cases.

1. Case 1: $i$ and $k$ are even, assuming $k>i$. According to the definition of $\theta_{i, j, k}$ in (32), the indices $j$ for which $\bar{\theta}_{i, j, k}$ are non-zero must satisfy the following two conditions

$$
\begin{aligned}
& j=\text { even number } \\
& k-i \leq j \leq k+i
\end{aligned}
$$

and the corresponding values for $\bar{\theta}_{i, j, k}$ are given by

$$
\bar{\theta}_{i, j, k}=\frac{i !(k-i+2 p) !}{p !(i-p) !(k-i+p) !}, \quad p=0, \cdots, i
$$

2. Case 2: $i$ is even and $k$ is odd, assuming $k>i$. According to the definition of $\theta_{i, j, k}$ in $(32)$, the indices $j$ for which $\bar{\theta}_{i, j, k}$ are non-zero must satisfy

$$
\begin{aligned}
& j=\text { odd number } \\
& k-i \leq j \leq k+i
\end{aligned}
$$

and the corresponding values for $\bar{\theta}_{i, j, k}$ are given by

$$
\bar{\theta}_{i, j, k}=\frac{i !(k-i+2 p) !}{p !(i-p) !(k-i+p) !}, \quad p=0, \cdots, i
$$

3. Case 3: $i$ and $k$ are odd, assuming $k>i$. According to the definition of $\theta_{i, j, k}$ in $(32)$, the indices $j$ for which $\bar{\theta}_{i, j, k}$ are non-zero must satisfy

$$
\begin{aligned}
& j=\text { even number } \\
& k-i \leq j \leq k+i
\end{aligned}
$$


and the corresponding values for $\bar{\theta}_{i, j, k}$ are given by

$$
\bar{\theta}_{i, j, k}=\frac{i !(k-i+2 p) !}{p !(i-p) !(k-i+p) !}, \quad p=0, \cdots, i
$$

4. Case 4: $i$ is odd and $k$ is even, assuming $k>i$. According to the definition of $\theta_{i, j, k}$ in $(32)$, the indices $j$ for which $\bar{\theta}_{i, j, k}$ are non-zero must satisfy

$$
\begin{gathered}
j=\text { odd number } \\
k-i \leq j \leq k+i
\end{gathered}
$$

and the corresponding values for $\bar{\theta}_{i, j, k}$ are given by

$$
\bar{\theta}_{i, j, k}=\frac{i !(k-i+2 p) !}{p !(i-p) !(k-i+p) !}, \quad p=0, \cdots, i
$$

The analysis of the values and patterns of the entries in the above four cases shows that they agree with the elements stated in Lemma 1 for the case of $k>i$. It can be shown, that analogous expressions for $i>k$ in the above four cases can be deduced by simply swapping $i$ and $k$. The case where $k=i$ follows from the above analysis. This proves the lemma.

The above four cases for $k>i$ and the other four cases for $k<i$ give us a clear idea about which coefficients $\boldsymbol{X}_{j}$ will be coupled with $\hat{\boldsymbol{G}}_{i}$ and the coupling factors, for a certain value of $k$. For example, assume that $i=3$ and $k=5$, then only coefficients $\boldsymbol{X}_{2}, \boldsymbol{X}_{4}, \boldsymbol{X}_{6}$ and $\boldsymbol{X}_{8}$ are multiplied by $\hat{\boldsymbol{G}}_{3}$ with coefficients, 1, 12,90 and 336 , respectively, as shown in the $6^{\text {th }}$ row of the corresponding matrix $\boldsymbol{K}_{3}$, assuming 
$M=9$, as follows:

$\boldsymbol{K}_{3}=\left[\begin{array}{cccccccccc}0 & 0 & 0 & 6 & 0 & 0 & 0 & 0 & 0 & 0 \\ 0 & 0 & 6 & 0 & 24 & 0 & 0 & 0 & 0 & 0 \\ 0 & 3 & 0 & 18 & 0 & 60 & 0 & 0 & 0 & 0 \\ 1 & 0 & 6 & 0 & 36 & 0 & 120 & 0 & 0 & 0 \\ 0 & 1 & 0 & 9 & 0 & 60 & 0 & 210 & 0 & 0 \\ 0 & 0 & 1 & 0 & 12 & 0 & 90 & 0 & 336 & 0 \\ 0 & 0 & 0 & 1 & 0 & 15 & 0 & 126 & 0 & 504 \\ 0 & 0 & 0 & 0 & 1 & 0 & 18 & 0 & 168 & 0 \\ 0 & 0 & 0 & 0 & 0 & 1 & 0 & 21 & 0 & 216 \\ 0 & 0 & 0 & 0 & 0 & 0 & 1 & 0 & 24 & 0\end{array}\right]$

\subsubsection{Lemma 2}

The lemma above provides a description for the matrices $\boldsymbol{K}_{i}$ that are used to generate the augmented matrices $\overline{\boldsymbol{G}}$ and $\overline{\boldsymbol{C}}$. It should be noted that those matrices, $\boldsymbol{K}_{i}$, are constant matrices that are problem-independent. The lemma 2 will then show that those matrices can be constructed recursively.

Lemma 2. Let $\boldsymbol{K}_{0}$ be an $(M+1) \times(M+1)$ identity matrix. Then $\boldsymbol{K}_{i}(i>0)$ is given by

$$
\boldsymbol{K}_{i}=\boldsymbol{A}_{L} \boldsymbol{K}_{i-1}+\boldsymbol{K}_{i-1} \boldsymbol{A}_{U}
$$


where

$$
\begin{aligned}
& \boldsymbol{A}_{L}=\left[\begin{array}{cccccc}
0 & 0 & 0 & \cdots & 0 & 0 \\
1 & 0 & 0 & \cdots & & 0 \\
0 & 1 & 0 & \cdots & & 0 \\
0 & 0 & 1 & 0 & \cdots & 0 \\
\vdots & 0 & \ddots & & & \\
0 & 0 & 0 & \cdots & 1 & 0
\end{array}\right] \\
& \boldsymbol{A}_{U}=\left[\begin{array}{ccccccc}
0 & 1 & 0 & 0 & \cdots & \cdots & 0 \\
0 & 0 & 2 & 0 & 0 & \cdots & 0 \\
0 & 0 & 0 & 3 & 0 & \cdots & 0 \\
\vdots & \ddots & & & & \vdots & 0 \\
0 & 0 & \cdots & & 0 & 0 & M \\
0 & 0 & \cdots & 0 & 0 & 0
\end{array}\right]
\end{aligned}
$$

Proof. To prove this lemma, we assume, without loss of generality, that both $k$ and $i$ are even numbers, with $k>i$. To prove the lemma, it will suffice to show that the $(k+1)^{\text {th }}$ row of $\boldsymbol{K}_{i+1}$ is identical to the $(k+1)^{\text {th }}$ row of $\boldsymbol{A}_{L} \boldsymbol{K}_{i}+\boldsymbol{K}_{i} \boldsymbol{A}_{U}$. To this end, we write down first the column indices of $(k+1)^{\text {th }}$ row of $\boldsymbol{K}_{i+1}$. The column indices of the nonzero entries of this row can be seen to be, guided by case 4 of the proof of Lemma 1 in Section 4.1.1, those $j+1$ for which $j$ is given by

$$
j=k-(i+1), k-(i+1)+2, \cdots, k+(i+1)
$$


and the numerical values for those entries are given by

$$
\frac{(i+1) !(k-(i+1)+2 p) !}{p !(i+1-p) !(k-(i+1)+p) !}, \quad p=0, \cdots, i+1
$$

We now take the $(k+1)^{\text {th }}$ row of of the matrix $\boldsymbol{A}_{L} \boldsymbol{K}_{i}+\boldsymbol{K}_{i} \boldsymbol{A}_{U}$ into consideration. It is clear that pre-multiplication by $\boldsymbol{A}_{L}$ shifts all rows down, while padding the first row with an all-zeros row. Also, post-multiplication by $\boldsymbol{A}_{U}$ shifts all columns to the right, scaling them by the column index (first column starts at $j=0$ ), while padding the first column with an all-zeros column.

First we consider the $(k+1)^{\text {th }}$ row in $\boldsymbol{A}_{L} \boldsymbol{K}_{i}$. Pre-multiplying $\boldsymbol{K}_{i}$ by $\boldsymbol{A}_{L}$ turns this row into a row whose non-zero entries occur at columns indices given by $j+1$, where

$$
j=k-i-1, k-i+1, \cdots, k+i-1
$$

which are all odd numbers, with numerical values given by

$$
\frac{i !(k-i+2 p-1) !}{p !(i-p) !(k-i+p-1) !}, \quad p=0, \cdots, i
$$

On the other hand, post-multiplication of $\boldsymbol{K}_{i}$ by $\boldsymbol{A}_{U}$ makes the nonzero entries of the $(k+1)^{\text {th }}$ row in $\boldsymbol{K}_{i} \boldsymbol{A}_{U}$ appear at column indices given by $j+1$ for the following values of $j$

$$
j=k-i+1, k-i+3, \cdots, k+i+1
$$

and with following numerical values,

$$
\begin{aligned}
\frac{i !(k-i+2 p) !}{p !(i-p) !(k-i+p) !} \times \overbrace{(k-i+2 p+1)}^{\text {scaling by } j} & \\
& =\frac{i !(k-i+2 p+1) !}{p !(i-p) !(k-i+p) !} \quad p=0, \cdots, i
\end{aligned}
$$


Adding the results from (60) and (62), it can be seen that the $(k+1)^{\text {th }}$ row in the matrix $\boldsymbol{A}_{L} \boldsymbol{K}_{i}+\boldsymbol{K}_{i} \boldsymbol{A}_{U}$ will have non-zero entries in columns whose indices are equal to $j+1$ for values of $j$ given by,

$$
j=k-(i+1), k-(i+1)+2, \cdots, k+(i+1)
$$

Those are indeed the same column indices of the non-zero entries in the $(k+1)^{\text {th }}$ row of $\boldsymbol{K}_{i+1}$, as can be seen from (57). Thus we proved that the non-zero entries in the $(k+1)^{\text {th }}$ row in both $\boldsymbol{A}_{L} \boldsymbol{K}_{i}+\boldsymbol{K}_{i} \boldsymbol{A}_{U}$ and $\boldsymbol{K}_{i+1}$ do occur at the same column indices. It remains to show that they also have the same numerical values to complete the proof of the lemma.

The non-zero value at the first entry, i.e., $j=k-(i+1)$, is obtained from (59) by substituting $p=0$, and turns out to be equal to 1 . This is indeed the first element in the $(k+1)^{\text {th }}$ row in $\boldsymbol{K}_{i+1}$.

The non-zero value at the last entry of, i.e. $j=k+(i+1)$, is obtained from (62) by substituting $p=i$ and is equal to $\frac{(k+i) !(k+i+1)}{k !}$ which can be simplified to $\frac{(k+i+1) !}{k !}$. Again, this is the last non-zero entry in the $(k+1)^{\text {th }}$ row of $\boldsymbol{K}_{i+1}$.

For other intermediate indices corresponding to $k-(i+1)<j<k+(i+1)$, the numerical values of those nonzero entries will be the sum of those given in (60) starting with $p=1$ and up to $p=i$, and those given in (62) starting with $p=0$ and up to $p=i-1$. We can perform this summation by replacing $p$ by $m+1$ in (60) and replacing $p$ by $m$ in (62), while letting $m$ range from $m=0$ and up to $m=i-1$. 
Proceeding with the following manipulation

$$
\begin{aligned}
& \frac{i !(k-i+2(m+1)-1) !}{(m+1) !(i-(m+1)) !(k-i+m) !}+\frac{i !(k-i+2 m+1) !}{m !(i-m) !(k-i+m) !} \\
& =\frac{i !(k-i+2 m+1) !}{(k-i+m) !}\left(\frac{i+1}{(m+1) !(i-m) !}\right)=\frac{(i+1) !(k-i+2 m+1) !}{(k-i+m) !(m+1) !(i-m) !} \\
& \quad m=0,1, \cdots, i-1
\end{aligned}
$$

Comparing the results obtained in (64) with the numerical entries of the $(k+1)^{\text {th }}$ row of $\boldsymbol{K}_{i+1}$ in (58) for $p=1,2, \cdots, i$ shows that they are identical. This completes the proof.

\subsubsection{Lemma 3}

Using the above two lemmas, the following lemma can be deduced.

Lemma 3. $\boldsymbol{K}_{i}$ can be expressed directly in terms of $\boldsymbol{A}_{L}$ and $\boldsymbol{A}_{U}$ using the binomial expansion,

$$
\boldsymbol{K}_{i}=\sum_{l=0}^{i} \frac{i !}{(i-l) ! l !} \boldsymbol{A}_{L}^{l} \boldsymbol{A}_{U}^{i-l}
$$

This lemma can be proved by recursively substituting in (54).

\subsection{The Case of Multi-random Variables}

The structure of the augmented matrices for the case of $d$-dimensional random space is obtained by the following result.

Lemma 4. Let $\boldsymbol{\beta}$ be a multi-index with d elements, $\beta_{i}(i=1, \cdots, d)$, then augmented 
matrices $\overline{\boldsymbol{G}}, \overline{\boldsymbol{C}}$ are obtained from

$$
\begin{aligned}
\overline{\boldsymbol{G}} & =\sum_{\boldsymbol{\beta}} \boldsymbol{K}_{\beta_{1}} \otimes \boldsymbol{K}_{\beta_{2}} \otimes \cdots \otimes \boldsymbol{K}_{\beta_{d}} \otimes \hat{\boldsymbol{G}}_{\boldsymbol{\beta}} \\
\overline{\boldsymbol{C}} & =\sum_{\boldsymbol{\beta}} \boldsymbol{K}_{\beta_{1}} \otimes \boldsymbol{K}_{\beta_{2}} \otimes \cdots \otimes \boldsymbol{K}_{\beta_{d}} \otimes \hat{\boldsymbol{C}}_{\boldsymbol{\beta}}
\end{aligned}
$$

where the summation is over all elements of $\boldsymbol{\beta}$ having $0 \leq \beta_{i} \leq Q$.

Proof. To prove this lemma, first rewriting (35)

$$
\begin{aligned}
& \sum_{\alpha}\left(\sum_{\beta} \hat{\boldsymbol{G}}_{\boldsymbol{\beta}} \bar{\Theta}_{\boldsymbol{\alpha}, \boldsymbol{\beta}, \boldsymbol{\gamma}}+s \sum_{\boldsymbol{\beta}} \hat{\boldsymbol{C}}_{\boldsymbol{\beta}} \bar{\Theta}_{\boldsymbol{\alpha}, \boldsymbol{\beta}, \boldsymbol{\gamma}}\right) \boldsymbol{X}_{\boldsymbol{\alpha}}(s)= \\
& \left\{\begin{array}{cc}
\boldsymbol{U} & \boldsymbol{\gamma}=\left[\begin{array}{cccc}
0 & 0 & \cdots & 0
\end{array}\right] \\
0 & \text { otherwise }
\end{array}\right.
\end{aligned}
$$

where

$$
\overline{\boldsymbol{\Theta}}_{\boldsymbol{\alpha}, \boldsymbol{\beta}, \boldsymbol{\gamma}}=\frac{\boldsymbol{\Theta}_{\boldsymbol{\alpha}, \boldsymbol{\beta}, \boldsymbol{\gamma}}}{\sqrt{2 \pi}^{d} \prod_{i=1}^{d} \gamma_{i} !}=\prod_{i=1}^{d} \bar{\theta}_{\alpha_{i}, \beta_{i}, \gamma_{i}}
$$

For the sake of simplifying the mathematical notation, we assume, without loss of generality, that the circuit under consideration leads to a scalar MNA formulation, i.e. $N=1$, and therefore $\hat{\boldsymbol{G}}_{\boldsymbol{\beta}}, \hat{\boldsymbol{C}}_{\boldsymbol{\beta}}$ are scalars, which could be denoted as $\hat{G}_{\boldsymbol{\beta}}$ and $\hat{C}_{\boldsymbol{\beta}}$, respectively. In this situation, the Galerkin-based PC approach to variability analysis will lead to a problem consisting of a linear system of equations in $(M+1)^{d}$ unknowns (Hermite coefficients). Furthermore, the Galerkin projection operation which was done for an arbitrary $\gamma$ and resulted in the system (68) will have to be repeated for all possible value of $\boldsymbol{\gamma}$, starting with an all-zeros multi-index $\gamma$ and up to the last multi-index $[M M \cdots M]$, and will result in a matrix of size $(M+1)^{d}$. It is possible to see that the $(|\gamma|+1)^{\text {th }}$ row, with $|\gamma|$ being defined by (19), will have the 
following structure

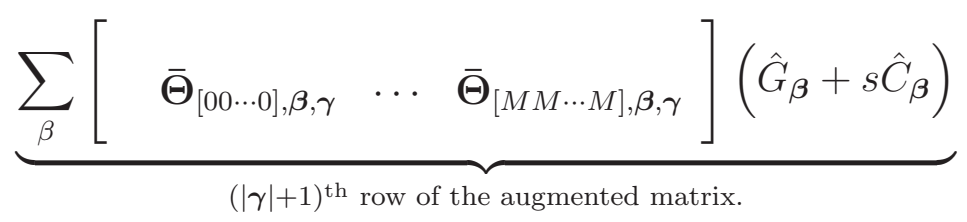

To recognize the above structure, let us recall a very important fact from the singledimensional analysis in Section 4.1.1. This fact states that a row constructed using

$$
\boldsymbol{\rho}_{\beta_{i}, \gamma_{i}}:=\left[\begin{array}{lllll}
\bar{\theta}_{0, \beta_{i}, \gamma_{i}} & \bar{\theta}_{1, \beta_{i}, \gamma_{i}} & \bar{\theta}_{2, \beta_{i}, \gamma_{i}} & \cdots & \bar{\theta}_{M, \beta_{i}, \gamma_{i}}
\end{array}\right]
$$

is the $\left(\gamma_{i}+1\right)^{\text {th }}$ row of the $\boldsymbol{K}_{\beta_{i}} \in \mathbb{R}^{(M+1) \times(M+1)}$ matrix derived in (65).

We can use the above-defined row to rewrite the augmented row in (70) as follows,

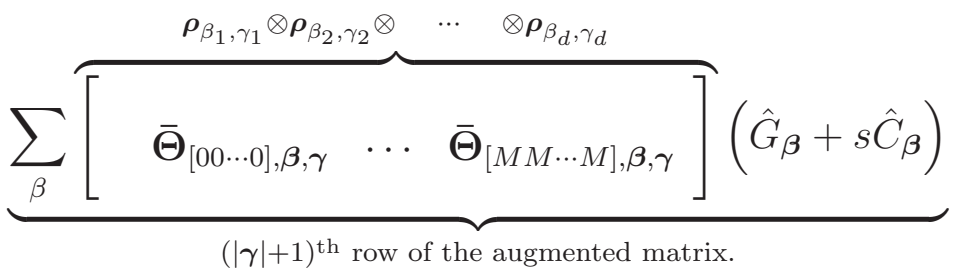

The entire matrix can be constructed by employing the Kronecker product

$$
\underbrace{\sum_{\beta}\left[\boldsymbol{K}_{\beta_{1}} \otimes \boldsymbol{K}_{\beta_{2}} \otimes \cdots \boldsymbol{K}_{\beta_{d}}\right]\left(\hat{G}_{\boldsymbol{\beta}}+s \hat{C}_{\boldsymbol{\beta}}\right)}_{\text {the augmented matrix. }}
$$

Generalizing the above result to $N>1$ will lead to augmented matrices of the form in (66) and (67), thereby completing the proof of this lemma. 


\subsection{Closed-form Representation of Augmented Matrices using Total Order}

Using the theory proposed in the previous sections of this chapter, we could provide analytical formulas for the structures of augmented system using the PC approach with total order.

For the sake of simplifying the mathematical notation, the superscript $*$ will be used here to distinguish the total lexicographic order from the tensor order. Thus, all the $\boldsymbol{\xi}$-dependent terms using total order are expanded as a series of Hermite polynomials in $\boldsymbol{\xi}$ as follows:

$$
\begin{aligned}
G^{*}(\boldsymbol{\xi}) & =\sum_{\boldsymbol{\beta}^{*}} \hat{\boldsymbol{G}}_{\boldsymbol{\beta}^{*}} \mathcal{H}_{\boldsymbol{\beta}^{*}}(\boldsymbol{\xi}) \\
\boldsymbol{C}^{*}(\boldsymbol{\xi}) & =\sum_{\boldsymbol{\beta}^{*}} \hat{\boldsymbol{C}}_{\boldsymbol{\beta}^{*}} \mathcal{H}_{\boldsymbol{\beta}^{*}}(\boldsymbol{\xi}) \\
\boldsymbol{X}^{*}(s, \boldsymbol{\xi}) & =\sum_{\boldsymbol{\alpha}^{*}} \boldsymbol{X}_{\boldsymbol{\alpha}^{*}}(s) \mathcal{H}_{\boldsymbol{\alpha}^{*}}(\boldsymbol{\xi})
\end{aligned}
$$

where $\boldsymbol{\alpha}^{*}$ and $\boldsymbol{\beta}^{*}$ are the $d$-dimensional multi-indices, which are corresponding to $\boldsymbol{\alpha}$ and $\boldsymbol{\beta}$, respectively:

$$
\begin{aligned}
& \boldsymbol{\alpha}^{*}=\left[\begin{array}{llll}
\alpha_{1}^{*} & \alpha_{2}^{*} & \cdots & \alpha_{d}^{*}
\end{array}\right] \\
& \boldsymbol{\beta}^{*}=\left[\begin{array}{llll}
\beta_{1}^{*} & \beta_{2}^{*} & \cdots & \beta_{d}^{*}
\end{array}\right]
\end{aligned}
$$

where $0 \leq \alpha_{i}^{*} \leq M, 0 \leq \beta_{i}^{*} \leq Q$ for $i=1, \cdots, d$; and $\sum_{i=1}^{d} \alpha_{i}^{*} \leq M, \sum_{i=1}^{d} \beta_{i}^{*} \leq Q$.

It should be noted again that, for the total lexicographic order, since its multiindex set is a sub-set of the corresponding tensor order as described in Chapter 2, particularly here we have $\boldsymbol{\alpha}^{*} \subset \boldsymbol{\alpha}$ and $\boldsymbol{\beta}^{*} \subset \boldsymbol{\beta}$, it is possible to construct the augmented 
system of DAEs with total order from the corresponding decoupling augmented system which uses the tensor order. To do this, we first define an order-selected operator $\boldsymbol{\tau}$, which is a matrix with $N_{\text {total }}$ row and $N_{\text {tensor }}$ column, in which the $i^{\text {th }}$ row has a single unity entry at a column index equal to the tensor-rank of the $i^{\text {th }}$ multi-index in the lexicographic order of $\boldsymbol{\alpha}_{*}$, assuming that rows and columns of $\boldsymbol{\tau}$ start at indices 0 .

Particularly, considering the example in Section 2.3.4, where $N_{\text {total }}=6$ and $N_{\text {tensor }}=9$, the order-selected operator $\boldsymbol{\tau}$ will be given by:

$$
\boldsymbol{\tau}=\left[\begin{array}{lllllllll}
1 & 0 & 0 & 0 & 0 & 0 & 0 & 0 & 0 \\
0 & 1 & 0 & 0 & 0 & 0 & 0 & 0 & 0 \\
0 & 0 & 1 & 0 & 0 & 0 & 0 & 0 & 0 \\
0 & 0 & 0 & 1 & 0 & 0 & 0 & 0 & 0 \\
0 & 0 & 0 & 0 & 1 & 0 & 0 & 0 & 0 \\
0 & 0 & 0 & 0 & 0 & 0 & 1 & 0 & 0
\end{array}\right]
$$

Thus, the augmented matrices with total order is obtained as follow

$$
\begin{aligned}
\overline{\boldsymbol{G}}^{*}= & {\left[\boldsymbol{\tau} \sum_{\boldsymbol{\beta}^{*}}\left(\boldsymbol{K}_{\beta_{1}^{*}} \otimes \boldsymbol{K}_{\beta_{2}^{*}} \otimes \cdots \otimes \boldsymbol{K}_{\beta_{d}^{*}}\right) \boldsymbol{\tau}^{\boldsymbol{T}}\right] \otimes \hat{\boldsymbol{G}}_{\boldsymbol{\beta}^{*}} } \\
\overline{\boldsymbol{C}}^{*}= & {\left[\boldsymbol{\tau} \sum_{\boldsymbol{\beta}^{*}}\left(\boldsymbol{K}_{\beta_{1}^{*}} \otimes \boldsymbol{K}_{\beta_{2}^{*}} \otimes \cdots \otimes \boldsymbol{K}_{\beta_{d}^{*}}\right) \boldsymbol{\tau}^{\boldsymbol{T}}\right] \otimes \hat{\boldsymbol{C}}_{\boldsymbol{\beta}_{*}} }
\end{aligned}
$$

This allows us to generate the following augmented system of DAEs using standard PC approach with total lexicographic order:

$$
\left(\bar{G}^{*}+s \bar{C}^{*}\right) \bar{X}^{*}(s)=\bar{U}^{*}
$$




\section{Chapter 5}

\section{The Proposed Decoupling Approach}

The previous chapter presented a closed-form characterization of the augmented matrices arising from the PC variability analysis. This chapter addresses the issue of factorizing those matrices with tensor order. The basic idea in the proposed approach is based on developing an alternative formulation for these augmented matrices that enables decoupling them into smaller matrices which can be factorized independently or in parallel. In a similar manner to the style adopted in the previous chapter, we will focus first on a single-dimensional random space, in Section 5.1, followed by the case of multiple random parameters, in Section 5.2. Next, Section 5.3 will present an approximate analysis to obtain an improved formulation for the decoupled PC method. Several numerical examples will also be provided for the accuracy validation of the proposed approach in the last section of this chapter.

\subsection{The Case of a Single Random Parameter}

For the sake of illustrating the main concept in the proposed decoupling approach,

assume that, instead of using $\boldsymbol{K}_{i}$, given by (65), we consider using an alternative 
matrix, denoted by $\check{\boldsymbol{K}}_{i}$, and computed from

$$
\check{\boldsymbol{K}}_{i}=\left(\boldsymbol{A}_{L}+\boldsymbol{A}_{U}\right)^{i}
$$

to construct the augmented matrices through the Kronecker product formulations in (41) and (42). An obvious objection to using $\check{\boldsymbol{K}}_{i}$ in place of $\boldsymbol{K}_{i}$ is that the obtained augmented matrices will deviate from the original augmented matrices $\overline{\boldsymbol{G}}$ and $\overline{\boldsymbol{C}}$. This is indeed the case, and, therefore, the $\check{\boldsymbol{K}}_{i}$-based augmented matrices will be denoted by $\check{\boldsymbol{G}}$ and $\check{\boldsymbol{C}}$ in order to distinguish them from $\overline{\boldsymbol{G}}$ and $\overline{\boldsymbol{C}}$. Thus, we have,

$$
\begin{aligned}
\check{\boldsymbol{G}} & =\sum_{i=0}^{Q} \check{\boldsymbol{K}}_{i} \otimes \hat{\boldsymbol{G}}_{i} \\
\check{\boldsymbol{C}} & =\sum_{i=0}^{Q} \check{\boldsymbol{K}}_{i} \otimes \hat{\boldsymbol{C}}_{i}
\end{aligned}
$$

Obviously, there are two important issues that need to be addressed before proceeding further:

1. The computational advantage of working with $\check{\boldsymbol{G}}, \check{\boldsymbol{C}}\left(\right.$ or $\left.\check{\boldsymbol{K}}_{i}\right)$ instead of $\overline{\boldsymbol{G}}, \overline{\boldsymbol{C}}$ (or $\boldsymbol{K}_{i}$ ), and

2. The proximity relation between a solution obtained by employing $\check{\boldsymbol{G}}, \check{\boldsymbol{C}}$ and the original exact solution obtained through $\overline{\boldsymbol{G}}, \overline{\boldsymbol{C}}$.

The rest of this section addresses the first question by reviewing the concept of the selector operator, before expanding the decoupling idea to the multi-random variable in the next section. The second question is then addressed in the Section 5.3.

\subsubsection{The Selector Operator}

To illustrate the potential computational advantages in using $\check{\boldsymbol{K}}_{i}$, we first stress the fact that the goal of variability analysis is to allow the designer to gauge the 
performance variability in only few, say $q$, variables of the circuit response. Those variables, which the designer deems of particular interest, can be grouped in a vector, denoted by $\boldsymbol{Y}(s) \in \mathbb{R}^{q}$. Typically, $\boldsymbol{Y}(s)$ is captured by defining a selector operator, $\boldsymbol{\psi}$ which is a matrix with $q$ columns and $N$ rows, in which each column has a single unity entry at a row index equal to the index of the variable of interest. Thus,

$$
\boldsymbol{Y}(s)=\boldsymbol{\psi}^{\top} \boldsymbol{X}(s)
$$

In order to extract the statistical properties of $\boldsymbol{Y}(s)$ using the PC approach, we will need its Hermite coefficients, which are given by $\boldsymbol{\psi}^{\top} \boldsymbol{X}_{\boldsymbol{\alpha}}(s)$ in the generalized case, for all the values of the multi-index $\boldsymbol{\alpha}$ taken into the truncated Hermite series. Let us

denote by $\overline{\boldsymbol{Y}}(s) \in \mathbb{R}^{q(M+1)^{d}}$ the vector whose $q(M+1)^{d}$ rows contain all the Hermite coefficients of those variables. It is not difficult to see that $\overline{\boldsymbol{Y}}(s)$ and $\overline{\boldsymbol{X}}(s)$ are related by

$$
\begin{aligned}
\overline{\boldsymbol{Y}}(s) & =\overline{\boldsymbol{\psi}}^{\top} \overline{\boldsymbol{X}}(s) \\
& =\overline{\boldsymbol{\psi}}^{\top}(\overline{\boldsymbol{G}}+s \overline{\boldsymbol{C}})^{-1} \overline{\boldsymbol{U}}
\end{aligned}
$$

where

$$
\overline{\boldsymbol{\psi}}=\boldsymbol{I}_{(M+1)^{d}} \otimes \boldsymbol{\psi}
$$

with $\boldsymbol{I}_{(M+1)^{d}}$ being identity matrix of size $(M+1)^{d}$.

\subsubsection{Approximate Decoupling Technique}

By employing the notion of the selector operator described above, the Hermite coefficients obtained from the alternative augmented matrices, $\check{\boldsymbol{G}}$ and $\check{\boldsymbol{C}}$, are selected. These selected coefficients in this case will be denoted by $\check{\boldsymbol{Y}}(s)$ to distinguish them from those obtained through the original augmented matrices. It then follows, that 


$$
\check{\boldsymbol{Y}}(s)=\overline{\boldsymbol{\psi}}^{\top}(\check{\boldsymbol{G}}+s \check{\boldsymbol{C}})^{-1} \overline{\boldsymbol{U}}
$$

Next, we use (84) and (85) in (89) while substituting for $\check{\boldsymbol{K}}_{i}$ by its eigendecomposition, which can be written as

$$
\check{\boldsymbol{K}}_{i}=\boldsymbol{V} \boldsymbol{\lambda}^{i} \boldsymbol{V}^{-1}
$$

where $\boldsymbol{\lambda}$ is a diagonal matrix whose diagonal elements are the eigenvalues of the matrix $\boldsymbol{K}_{1}=\boldsymbol{A}_{L}+\boldsymbol{A}_{U}$ and $\boldsymbol{V}$ is the matrix of eigenvectors of $\check{\boldsymbol{K}}_{i}$. Due to the fact that $\check{\boldsymbol{K}}_{i}$ is given by $\left(\boldsymbol{A}_{L}+\boldsymbol{A}_{U}\right)^{i}$, its matrix of eigenvectors is always independent of $i$. This means that all matrices $\check{\boldsymbol{K}}_{i}$ will share the same matrix of eigenvectors $\boldsymbol{V}$, which is the matrix of eigenvectors of $\boldsymbol{K}_{1}$. This feature allows us to factor the matrix $\boldsymbol{V}$ out of the summation over $i$ and transform (89), using the properties of the Kronecker operator, into the following form:

$$
\check{\boldsymbol{Y}}(s)=\overline{\boldsymbol{\psi}}^{\top} \boldsymbol{\Gamma}\left(\sum_{i=0}^{Q}\left(\boldsymbol{\lambda}^{i} \otimes \hat{\boldsymbol{G}}_{i}+s \boldsymbol{\lambda}^{i} \otimes \hat{\boldsymbol{C}}_{i}\right)\right)^{-1} \boldsymbol{\Gamma}^{-1} \overline{\boldsymbol{U}}
$$

where

$$
\boldsymbol{\Gamma}=\boldsymbol{V} \otimes \boldsymbol{I}_{N}
$$

with $\boldsymbol{I}_{N}$ being identity matrix of size $N$.

The above expression for $\check{\boldsymbol{Y}}(s)$ can be expanded, using the definition of the Kronecker product, to show that, 


$$
\check{\boldsymbol{Y}}(s)=\overline{\boldsymbol{\psi}}^{\top} \boldsymbol{\Gamma}\left[\begin{array}{cccc}
\boldsymbol{L}_{0} & \mathbf{0} & \cdots & \mathbf{0} \\
0 & \boldsymbol{L}_{1} & \cdots & \mathbf{0} \\
\vdots & 0 & \ddots & \vdots \\
\vdots & 0 & 0 & \boldsymbol{L}_{M}
\end{array}\right] \boldsymbol{\Gamma}^{-1} \overline{\boldsymbol{U}}
$$

where,

$$
\boldsymbol{L}_{k}=\left(\sum_{i=0}^{Q}\left(\lambda_{k}\right)^{i}\left(\hat{\boldsymbol{G}}_{i}+s \hat{\boldsymbol{C}}_{i}\right)\right)^{-1}
$$

and $\lambda_{k}$ is the $k^{\text {th }}$ eigenvalue of the matrix $\boldsymbol{K}_{1}$.

In (93), it can be clearly seen that the matrix which needs to be factorized is block diagonal, whose blocks are of size $N$. Those blocks can be factorized independently of each other or in parallel on suitable multi-core platform, effectively leading to decoupling the large augmented matrix into smaller matrices. This approximate framework of decoupling the original augmented matrix into several $N$-sized blocks is what provides the computational advantage in the proposed technique.

It is important, before generalizing the above technique to the case of more than one random parameter, to stress the fact that there is no noticeable computational efforts (beyond the factorization of the diagonal blocks) involved in (91). More specifically, the matrices $\boldsymbol{\Gamma}$ or $\boldsymbol{\Gamma}^{-1}$ do not need to be computed since they are based on the matrix $\boldsymbol{V}$ or $\boldsymbol{V}^{-1}$ which is problem-independent, and therefore can be made available offline. Furthermore, the operations of $\overline{\boldsymbol{\psi}}^{\top} \boldsymbol{\Gamma}$ (or $\boldsymbol{\Gamma}^{-1} \overline{\boldsymbol{U}}$ ) can be carried out by scaling one or more rows of $\boldsymbol{V} \in \mathbb{R}^{(M+1) \times(M+1)}$ (respectively, columns of $\boldsymbol{V}^{-1}$ ) and spreading the entries appropriately in vector of size $(M+1) N$. 


\subsection{The Case of Multi-random Parameters}

Generalizing the decoupling idea is carried out by employing $\check{\boldsymbol{K}}_{\beta_{i}}$ in building the augmented matrices in (66) and (67) instead of $\boldsymbol{K}_{\beta_{i}}$, for all $i=1, \cdots, d$. It can be shown that proceeding along the above analysis steps will transform (89) into the following system

$$
\check{\boldsymbol{Y}}(s)=\overline{\boldsymbol{\psi}}^{\top} \overline{\boldsymbol{\Gamma}}\left(\sum_{\boldsymbol{\beta}} \boldsymbol{D}_{\boldsymbol{\beta}}(\boldsymbol{\lambda}) \otimes \hat{\boldsymbol{G}}_{\boldsymbol{\beta}}+s \sum_{\boldsymbol{\beta}} \boldsymbol{D}_{\boldsymbol{\beta}}(\boldsymbol{\lambda}) \otimes \hat{\boldsymbol{C}}_{\boldsymbol{\beta}}\right)^{-1} \overline{\boldsymbol{\Gamma}}^{-1} \overline{\boldsymbol{U}}
$$

where

$$
\boldsymbol{D}_{\boldsymbol{\beta}}(\boldsymbol{\lambda})=\boldsymbol{\lambda}^{\beta_{1}} \otimes \boldsymbol{\lambda}^{\beta_{2}} \otimes \cdots \otimes \boldsymbol{\lambda}^{\beta_{d}}
$$

and

$$
\overline{\boldsymbol{\Gamma}}=\underbrace{\boldsymbol{V} \otimes \boldsymbol{V} \otimes \cdots \otimes \boldsymbol{V}}_{d \text { times }} \otimes \boldsymbol{I}_{N}
$$

It should be stressed again here, that the matrix to be factorized in this new formulation is block diagonal, with decoupled blocks that can be factorized independently. In addition, the transformation matrix $\bar{\Gamma}$ is problem-independent, and, therefore, does not need to be computed. Furthermore, the typical structure of the matrices $\overline{\boldsymbol{U}}$ and $\overline{\boldsymbol{\psi}}$ enables computing $\overline{\boldsymbol{\psi}}^{\top} \overline{\boldsymbol{\Gamma}}$ and $\overline{\boldsymbol{\Gamma}}^{-1} \overline{\boldsymbol{U}}$ by simply selecting, or scaling, few rows and columns of $\overline{\boldsymbol{\Gamma}}$ and $\overline{\boldsymbol{\Gamma}}^{-1}$. Those rows or columns are obtained, in turn, from the Kronecker product of rows and columns of $\boldsymbol{V}$ and $\boldsymbol{V}^{-1}$ and spread in a vector of dimension $(M+1)^{d} N$.

\subsection{An Improved Decoupled Formulation}

This section considers the relation between the exact Hermite coefficients, $\overline{\boldsymbol{Y}}(s)$,

and the approximate $\check{\boldsymbol{Y}}(s)$ obtained through the decoupled-based formulation. In 
addition, it presents an alternative formulation that enhances the proximity between them. The development in this section will be guided by the following two main observations.

1. The first observation is that $\check{\boldsymbol{Y}}(s)$ deviates from $\overline{\boldsymbol{Y}}(s)$ in as much as $\check{\boldsymbol{K}}_{\beta_{i}}$ departs from the original $\boldsymbol{K}_{\beta_{i}}$, and

2. the key idea that enabled the decoupling approach, as shown from the analysis leading up to (95), was the fact that eigenvectors of the matrices $\check{\boldsymbol{K}}_{\beta_{i}}$ are actually independent from the sub-index $\beta_{i}$.

Under the first observation, we further note that $\check{\boldsymbol{K}}_{i}$, described by (83), and $\boldsymbol{K}_{i}$, given by (65), could have been identical, had the matrices $\boldsymbol{A}_{L}$ and $\boldsymbol{A}_{U}$ been commuting matrices, i.e. if their Lie Bracket operation, $\left[\boldsymbol{A}_{U}, \boldsymbol{A}_{L}\right]$, defined by

$$
\left[\boldsymbol{A}_{U}, \boldsymbol{A}_{L}\right]:=\boldsymbol{A}_{U} \boldsymbol{A}_{L}-\boldsymbol{A}_{L} \boldsymbol{A}_{U}
$$

vanishes. Unfortunately, this is not the case.

We also note that seeking an improved matrix to build the augmented formulation must always take into account the second observation to maintain the decoupling feature in the augmented matrices.

The approach used to obtain an improved matrix will be illustrated through considering the difference between $\check{\boldsymbol{K}}_{i}$ and $\boldsymbol{K}_{i}$. We first note that $\check{\boldsymbol{K}}_{i}=\boldsymbol{K}_{i}$ for $i=0,1$. Therefore, we start investigating the difference matrix, for $i=2$, i.e. $\check{\boldsymbol{K}}_{2}-\boldsymbol{K}_{2}$, which 
is given by

$$
\check{\boldsymbol{K}}_{2}-\boldsymbol{K}_{2}=\left[\begin{array}{cccc}
1 & 0 & \cdots & 0 \\
0 & 1 & \cdots & 0 \\
\vdots & 0 & \ddots & 0 \\
0 & \cdots & & -M
\end{array}\right]
$$

The above result suggests that an improved, in the sense of being closer to the original $\boldsymbol{K}_{2}$, matrix, denoted $\tilde{\boldsymbol{K}}_{2}$, can be defined using

$$
\tilde{\boldsymbol{K}}_{2}=\left(\boldsymbol{A}_{U}+\boldsymbol{A}_{L}\right)^{2}-\boldsymbol{I}
$$

which still has the same eigenvectors matrix $\boldsymbol{V}$ of $\boldsymbol{A}_{L}+\boldsymbol{A}_{U}$, as required in the above second observation. The new matrix reduces the non-zero entries in the difference matrix, $\tilde{\boldsymbol{K}}_{2}-\boldsymbol{K}_{2}$ to only one,

$$
\tilde{\boldsymbol{K}}_{2}-\boldsymbol{K}_{2}=\left[\begin{array}{cccc} 
& & & \\
0 & 0 & \cdots & 0 \\
0 & 0 & \cdots & 0 \\
\vdots & 0 & \ddots & 0 \\
0 & \cdots & & -M-1
\end{array}\right]
$$

In a similar manner, improved matrices, for $i=, 3,4,5$, and 6 were derived and were found to be sufficiently accurate for the purposes of variability analysis. Those 
matrices are shown in (102)-(105):

$$
\begin{aligned}
\tilde{\boldsymbol{K}}_{3} & =\left(\boldsymbol{A}_{U}+\boldsymbol{A}_{L}\right)^{3}-3\left(\boldsymbol{A}_{U}+\boldsymbol{A}_{L}\right) \\
\tilde{\boldsymbol{K}}_{4} & =\left(\boldsymbol{A}_{U}+\boldsymbol{A}_{L}\right)^{4}-6\left(\boldsymbol{A}_{U}+\boldsymbol{A}_{L}\right)^{2}+3 \boldsymbol{I} \\
\tilde{\boldsymbol{K}}_{5} & =\left(\boldsymbol{A}_{U}+\boldsymbol{A}_{L}\right)^{5}-10\left(\boldsymbol{A}_{U}+\boldsymbol{A}_{L}\right)^{3}+15\left(\boldsymbol{A}_{U}+\boldsymbol{A}_{L}\right) \\
\tilde{\boldsymbol{K}}_{6} & =\left(\boldsymbol{A}_{U}+\boldsymbol{A}_{L}\right)^{6}-15\left(\boldsymbol{A}_{U}+\boldsymbol{A}_{L}\right)^{4}+45\left(\boldsymbol{A}_{U}+\boldsymbol{A}_{L}\right)^{2}-15 \boldsymbol{I}
\end{aligned}
$$

For example, using a component-wise norm operator to gauge the proximity between the improved $\tilde{\boldsymbol{K}}_{6}$ and the exact $\boldsymbol{K}_{6}$, we find that,

$$
\frac{\left|\tilde{\boldsymbol{K}}_{6}-\boldsymbol{K}_{6}\right|}{\left|\boldsymbol{K}_{6}\right|}=0.1003
$$

which is better, in the sense of being more accurate, than $\check{\boldsymbol{K}}_{6}$ of (83) for $i=6$, as can be seen from the following

$$
\frac{\left|\check{\boldsymbol{K}}_{6}-\boldsymbol{K}_{6}\right|}{\left|\boldsymbol{K}_{6}\right|}=0.1930
$$

In addition, those improved matrices share the same eigenvectors matrix, $\boldsymbol{V}$, and therefore serve as better approximants to $\boldsymbol{K}_{i}$ than $\check{\boldsymbol{K}}_{i}$, without losing the decoupling feature of the latter.

The adoption of those matrices to construct the augmented matrices will modify the decoupled structure (95) to take the following form

$$
\tilde{\boldsymbol{Y}}(s)=\overline{\boldsymbol{\psi}}^{\top} \overline{\boldsymbol{\Gamma}}\left(\sum_{\boldsymbol{\beta}} \boldsymbol{F}_{\boldsymbol{\beta}}(\boldsymbol{\lambda}) \otimes \hat{\boldsymbol{G}}_{\boldsymbol{\beta}}+s \sum_{\boldsymbol{\beta}} \boldsymbol{F}_{\boldsymbol{\beta}}(\boldsymbol{\lambda}) \otimes \hat{\boldsymbol{C}}_{\boldsymbol{\beta}}\right)^{-1} \overline{\boldsymbol{\Gamma}}^{-1} \overline{\boldsymbol{U}}
$$

where

$$
\boldsymbol{F}_{\boldsymbol{\beta}}(\boldsymbol{\lambda})=F_{\beta_{1}}(\boldsymbol{\lambda}) \otimes F_{\beta_{2}}(\boldsymbol{\lambda}) \otimes \cdots F_{\beta_{d}}(\boldsymbol{\lambda})
$$


and

$$
\begin{aligned}
& F_{0}(\lambda)=1 \\
& F_{1}(\lambda)=\lambda \\
& F_{2}(\lambda)=\lambda^{2}-1 \\
& F_{3}(\lambda)=\lambda^{3}-3 \lambda \\
& F_{4}(\lambda)=\lambda^{4}-6 \lambda^{2}+3 \\
& F_{5}(\lambda)=\lambda^{5}-10 \lambda^{3}+15 \lambda \\
& F_{6}(\lambda)=\lambda^{6}-15 \lambda^{4}+45 \lambda^{2}-15
\end{aligned}
$$

\subsection{Numerical Validation}

In this section, simulation results of the proposed algorithm are presented and compared with those obtained from the standard PC and Monte Carlo based simulations. Basing on the sensitivity of the uncertain parameters, the output response will be expanded differently for different random variable, in terms of Hermite series. Particularly, in the following examples, $\boldsymbol{M}=\left[\begin{array}{llll}p_{1} & p_{2} & \cdots & p_{d}\end{array}\right]^{T}$ is denoted as the vector whose the value of $p_{i}$ is the highest truncated Hermite order of the circuit response expansion for the $i^{\text {th }}$ random variable $(i=1,2, \cdots, d)$.

\subsubsection{Example 1}

In this example, a macromodel for two coupled transmission lines, shown in Fig. 2, has been used to test the proposed algorithm in comparison to the standard PC method. Each TL here has a length of $10 \mathrm{~cm}$ and its macromodel is constructed using 100 sections of lumped RLGC segmentation. The excitation source in the circuit is an AC voltage source with $1 \mathrm{~V}$ amplitude at the near-end of the first line while the 
remaining line is kept quiet.

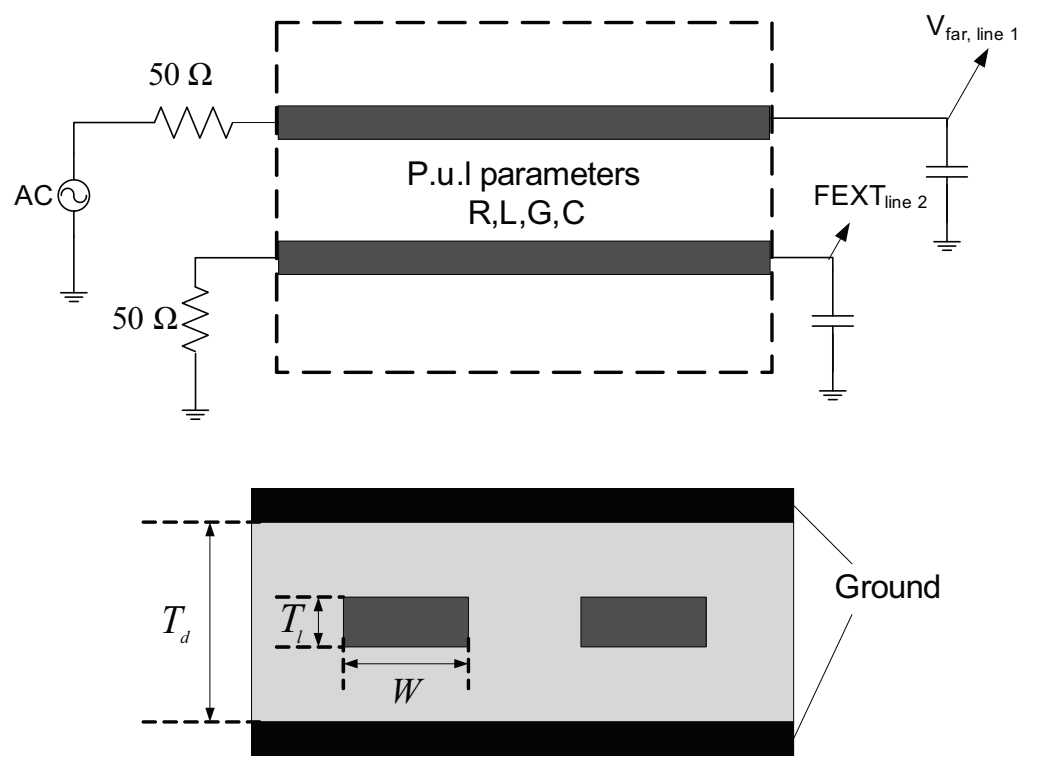

Figure 2: Two coupled transmission-line circuit (top panel) and its cross-section (bottom panel) of Example 1.
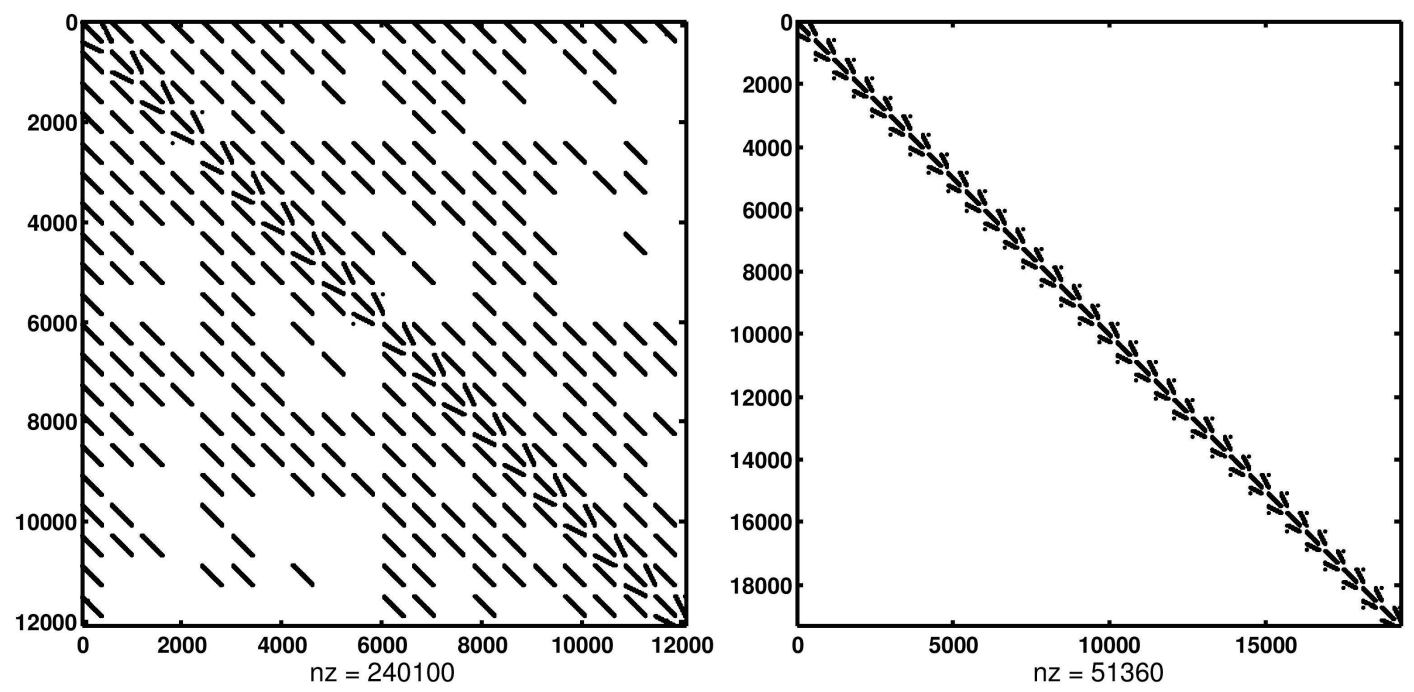

Figure 3: Sparsity patterns of augmented coupled matrix $\bar{G}^{*}$ with total order (left hand side) and augmented decoupled matrix $\check{G}$ with tensor order (right hand side)

The sources of uncertainty in this circuit are assumed to arise from the thickness of dielectric $T_{d}$, the thickness of the conductor $T_{l}$ and its width $W$. Those uncertain 


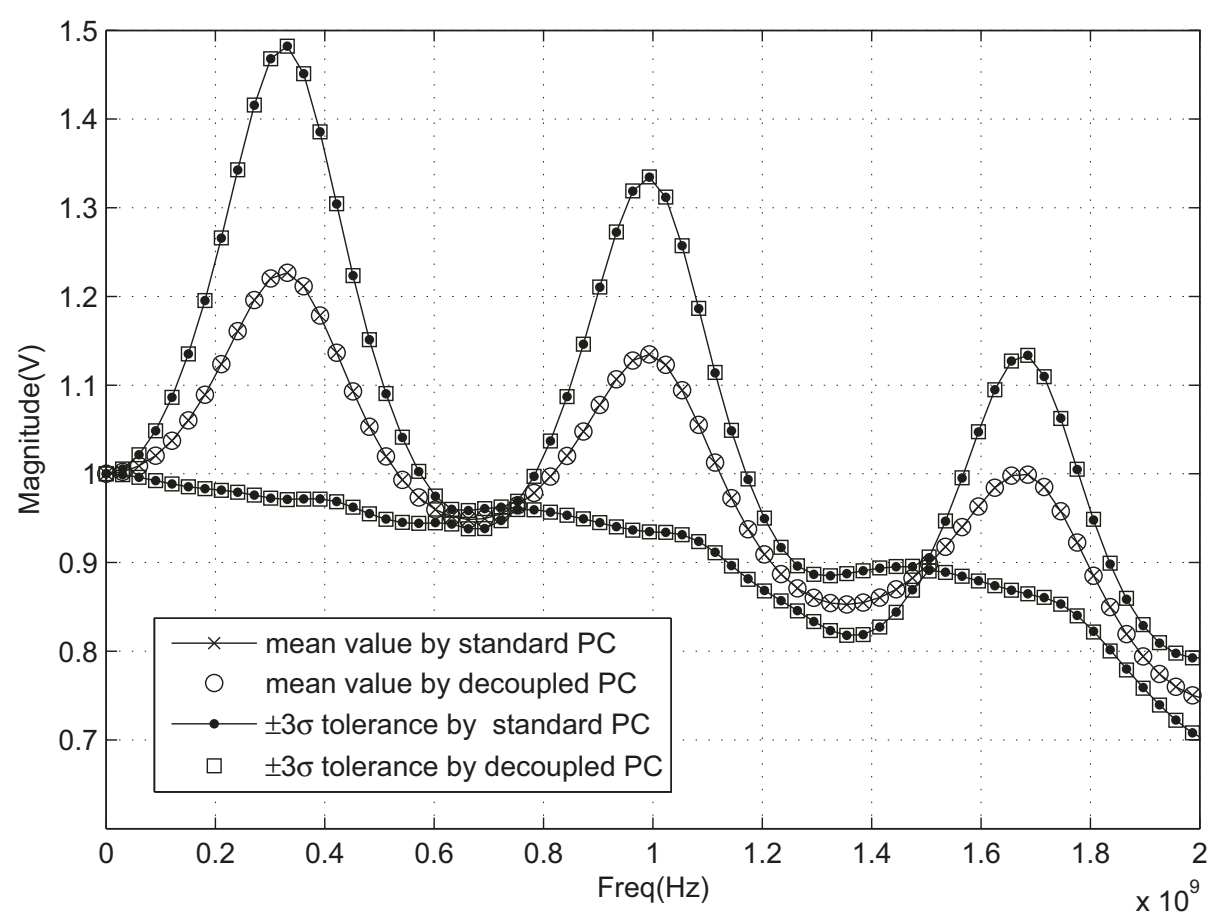

Figure 4: Statistical analysis for the magnitude of the far-end voltage of line 1 (example 1).

physical parameters were expressed as functions of 3 independent normalized Gaussian random variable (with zero mean and unit variance) $\xi_{i}(i=1,2,3)$, as follows,

$$
\begin{aligned}
W & =\bar{W}\left(1+\sigma \xi_{1}\right) \\
T_{l} & =\bar{T}_{l}\left(1+\sigma \xi_{2}\right) \\
T_{d} & =\bar{T}_{d}\left(1+\sigma \xi_{3}\right)
\end{aligned}
$$

where $\sigma=0.1$ is the normalized standard deviation, while $\bar{W}, \bar{T}_{l}$ and $\bar{T}_{d}$ are the nominal values of the parameters, given by, $\bar{W}=5 \mathrm{mil}, \bar{T}_{l}=0.6 \mathrm{mil}$ and $\bar{T}_{d}=20 \mathrm{mil}$.

To visualize the potential computational advantage of the proposed approach, Fig. 3 presents the sparsity pattern of the augmented coupled matrix, denoted by $\bar{G}^{*}$ (left), using standard PC with third-order expansion $(M=3)$, and the sparsity pattern of the proposed augmented matrix $\check{G}$ (right) using the tensor order where 


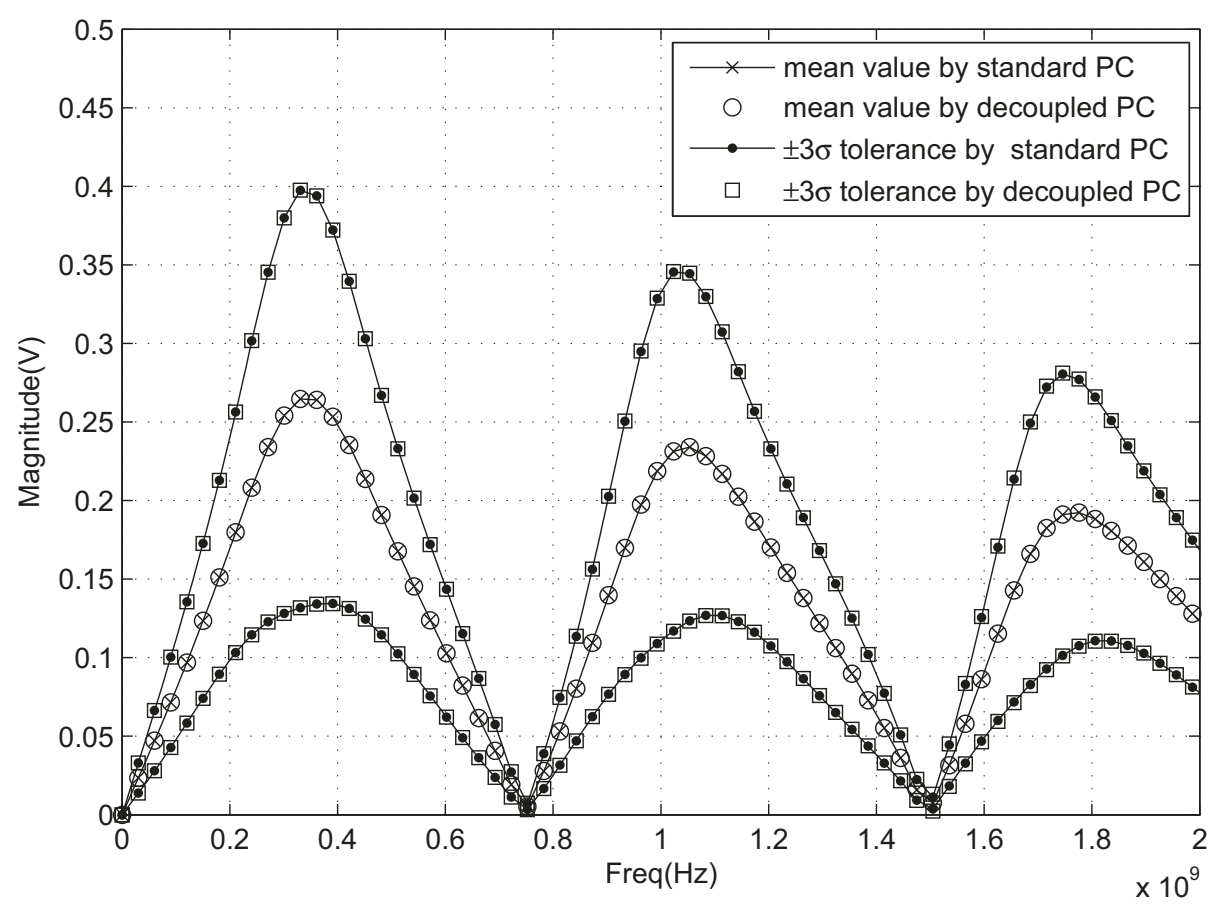

Figure 5: Statistical analysis for the magnitude of the far-end cross-talk of line 2 (example 1).

$\boldsymbol{M}=\left[\begin{array}{lll}3 & 1 & 3\end{array}\right]^{T}$. As can be seen, the matrix $\check{\boldsymbol{G}}$ after decoupling is transformed into block diagonal, whose diagonal blocks can be factorized independently.

Figs. 4 and 5 show the mean value and the $\pm 3 \sigma$ tolerances using both the decoupling algorithm and the regular coupled PC approach for the far-end voltage magnitudes of lines 1 and 2, respectively. As can be seen, the mean value and the $3 \sigma$ boundaries using both approaches match accurately.

\subsubsection{Example 2}

In this example, the proposed algorithm is implemented to characterize the variability of the eight coupled transmission line structure shown in Fig. 6. Each TL has a length of $10 \mathrm{~cm}$ and its macromodel is constructed using 100 sections of lumped RLGC segmentation. The first of every two lines is excited by an $\mathrm{AC}$ voltage source with $1 \mathrm{~V}$ amplitude at its near-end while the remaining lines are kept quiet. 

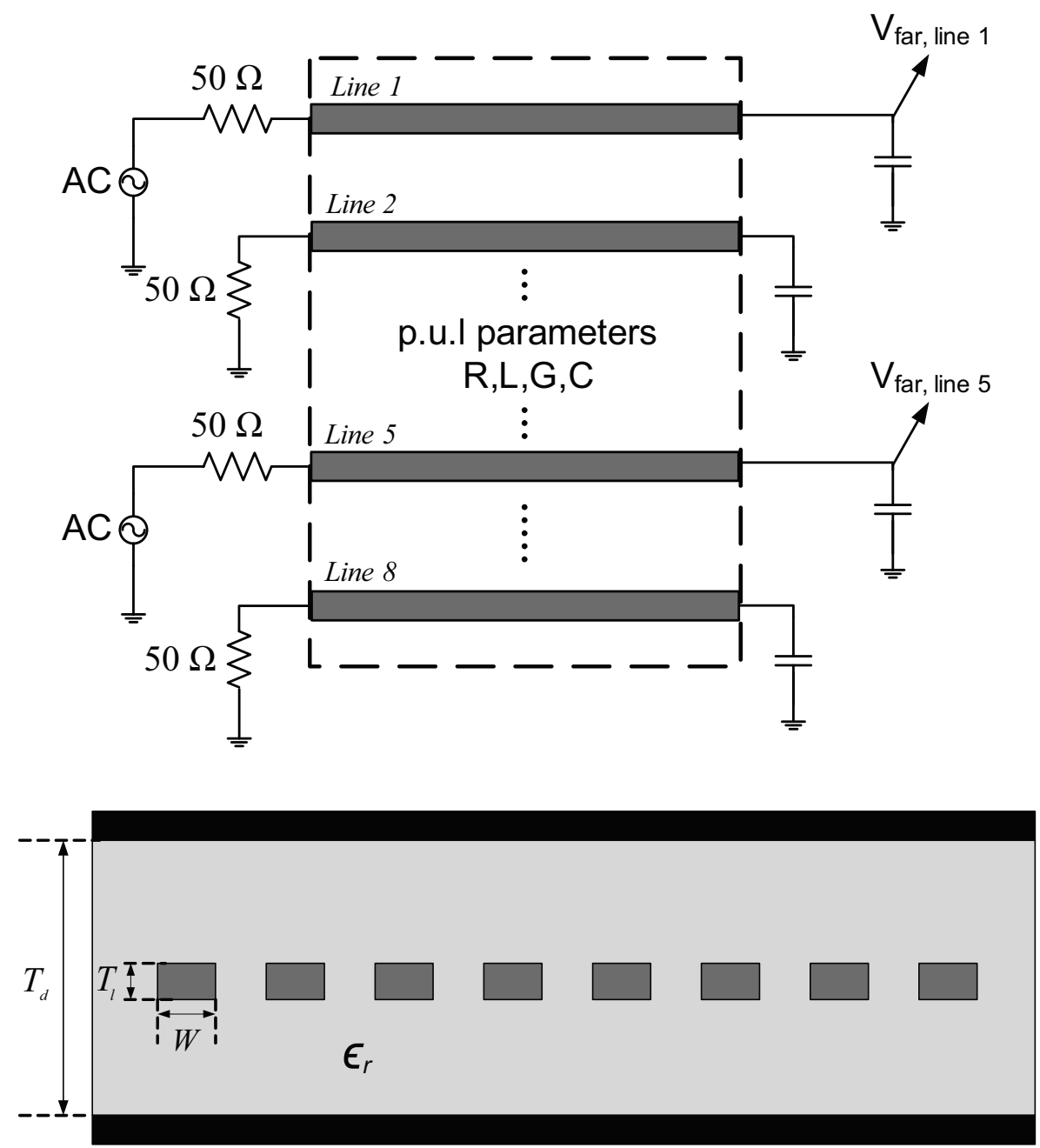

Figure 6: Eight coupled transmission-line circuit (top panel) and its cross-section (bottom panel) of example 2 .

The sources of uncertainty in this circuit are assumed to arise from the thickness of dielectric $T_{d}$, and its relative permittivity $\epsilon_{r}$, the thickness of the conductor $T_{l}$ and its width $W$. Those uncertain physical parameters were expressed as functions of 4 independent normalized Gaussian random variable (with zero mean and unit 
variance) $\xi_{i}$, for $i=1, \cdots, 4$, as shown next,

$$
\begin{aligned}
W & =\bar{W}\left(1+\sigma \xi_{1}\right) \\
T_{l} & =\bar{T}_{l}\left(1+\sigma \xi_{2}\right) \\
T_{d} & =\bar{T}_{d}\left(1+\sigma \xi_{3}\right) \\
\epsilon_{r} & =\bar{\epsilon}_{r}\left(1+\sigma \xi_{4}\right)
\end{aligned}
$$

where $\sigma=0.1$ is the normalized standard deviation, while $\bar{W}, \bar{T}_{l}, \bar{T}_{d}$ and $\bar{\epsilon}_{r}$ are the nominal values of the parameters, given by, $\bar{W}=5 \mathrm{mil}, \bar{T}_{l}=0.6 \mathrm{mil}, \bar{T}_{d}=20 \mathrm{mil}$ and $\overline{\epsilon_{r}}=4$.

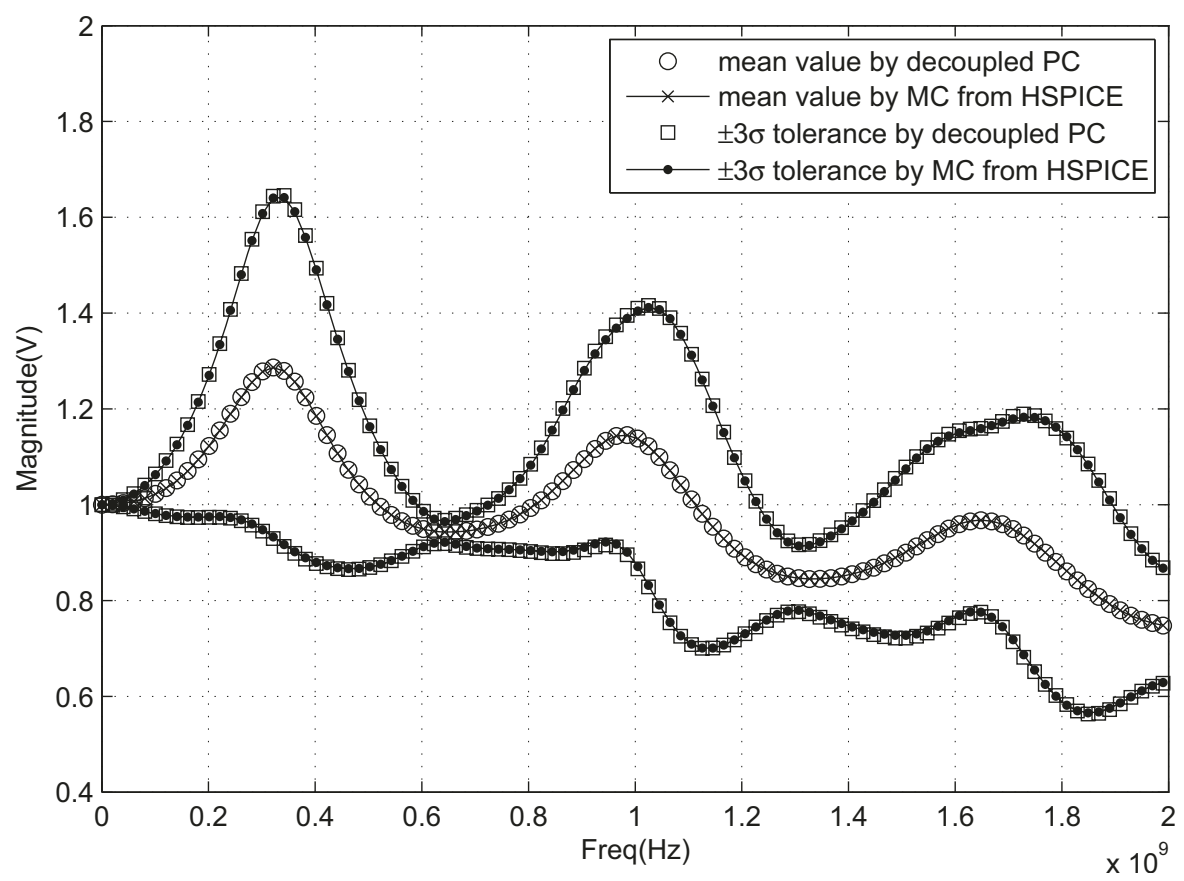

Figure 7: Statistical analysis for the magnitude of the far-end voltage of line 1 (example 2).

Figs. 7-10 show the mean value and the $\pm 3 \sigma$ tolerances of the magnitude of far-end voltages of four active lines obtained by both the proposed decoupled PC approach and Monte-Carlo method using $\mathrm{HSPICE}^{\circledR}$. Those figures demonstrate a 


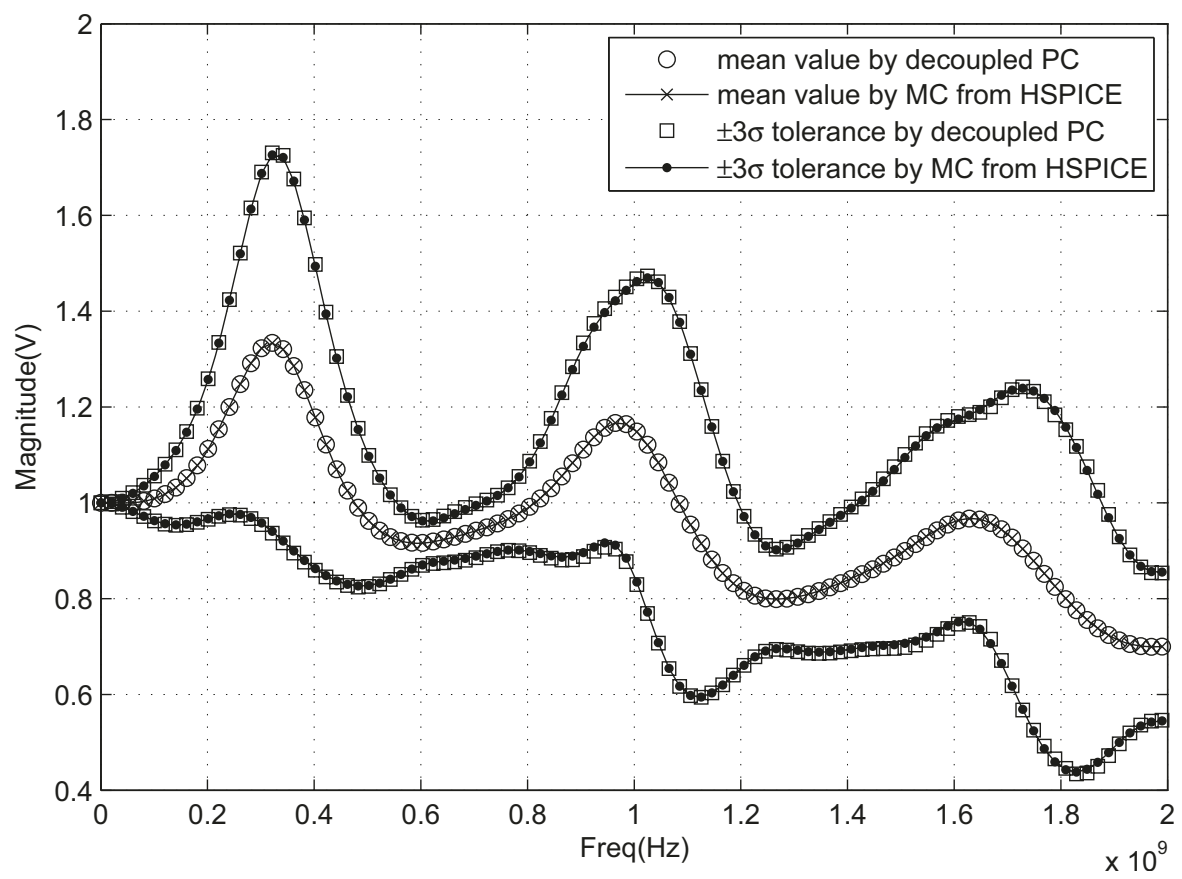

Figure 8: Statistical analysis for the magnitude of the far-end voltage of line 3 (example 2).

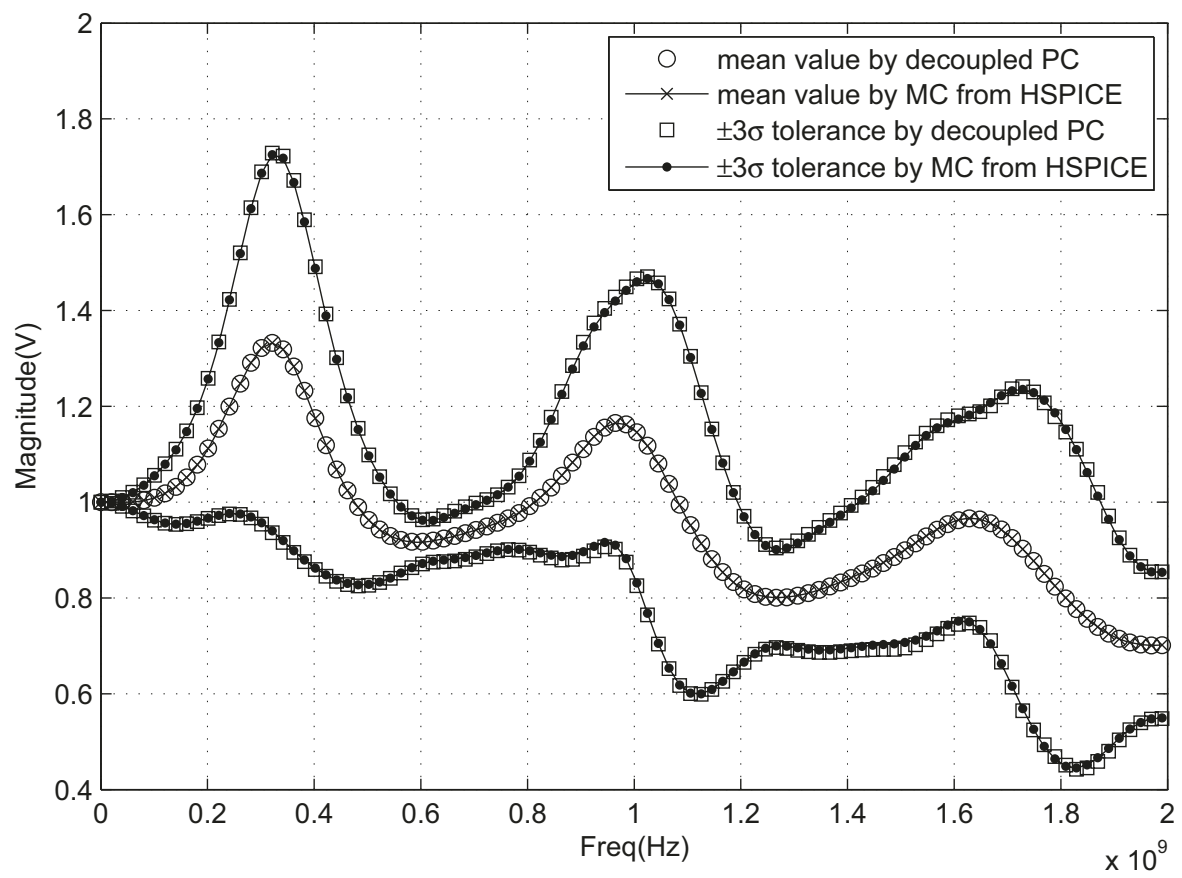

Figure 9: Statistical analysis for the magnitude of the far-end voltage of line 5 (example 2). 


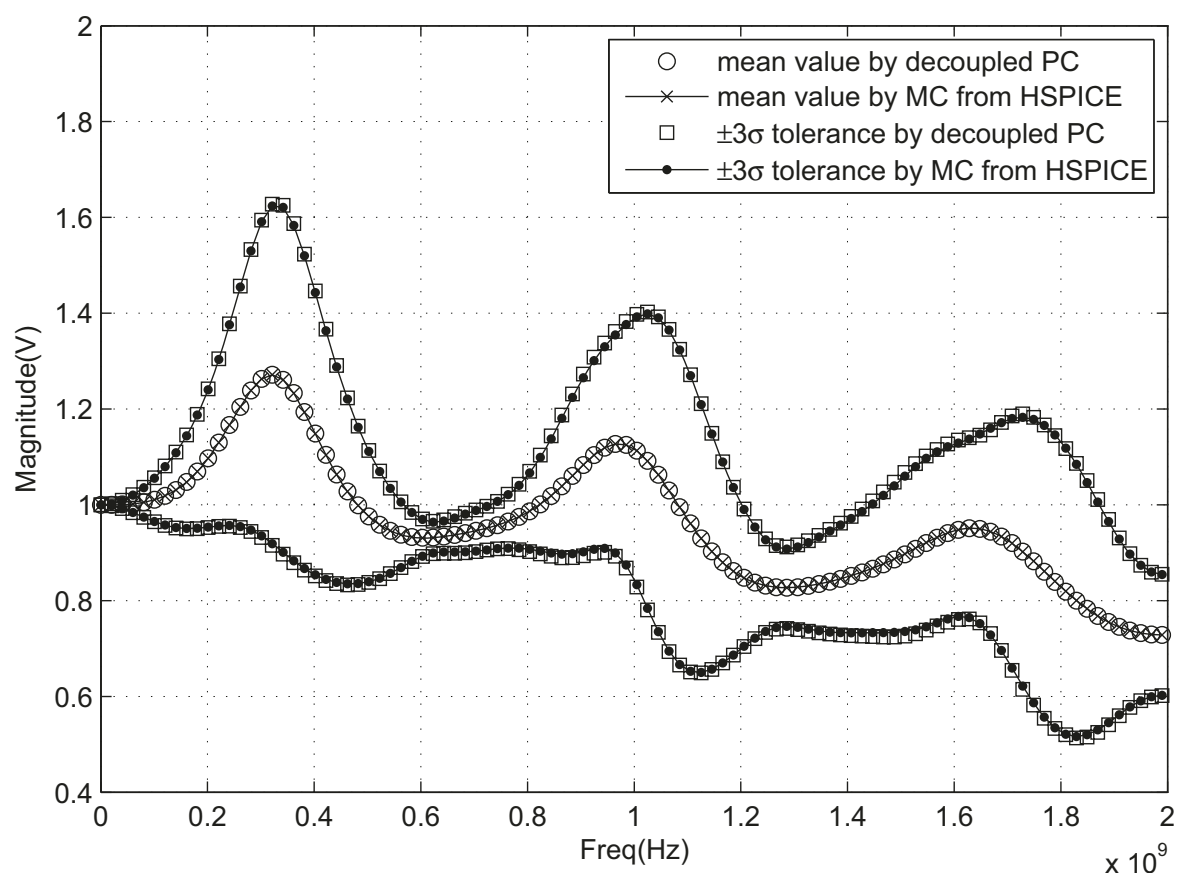

Figure 10: Statistical analysis for the magnitude of the far-end voltage of line 7 (example 2).

good agreement between the proposed approach and the classical MC approach.

\subsubsection{Example 3}

This example considers the task of performing yield analysis on the circuit of active low-pass filter shown in Fig. 11 [21]. The frequency-domain response of the output voltage $V_{\text {out }}$, due to a $1 \mathrm{~V}$ amplitude at the input, was required to satisfy the following specifications.

- A pass-band that extends from 0 to $3400 \mathrm{~Hz}$ with $0.2 \mathrm{~dB}$ ripple.

- A stop-band starting from $3850 \mathrm{~Hz}-\infty$ with minimum attenuation of $50 \mathrm{~dB}$.

The Operational Amplifiers (OpAmps) of the circuit were all modelled with the circuit shown in Fig. 12. The circuit parameters that represented the design uncertainty in this example were taken to be $R_{1}, R_{21}, C_{9}$, with each one being represented with 


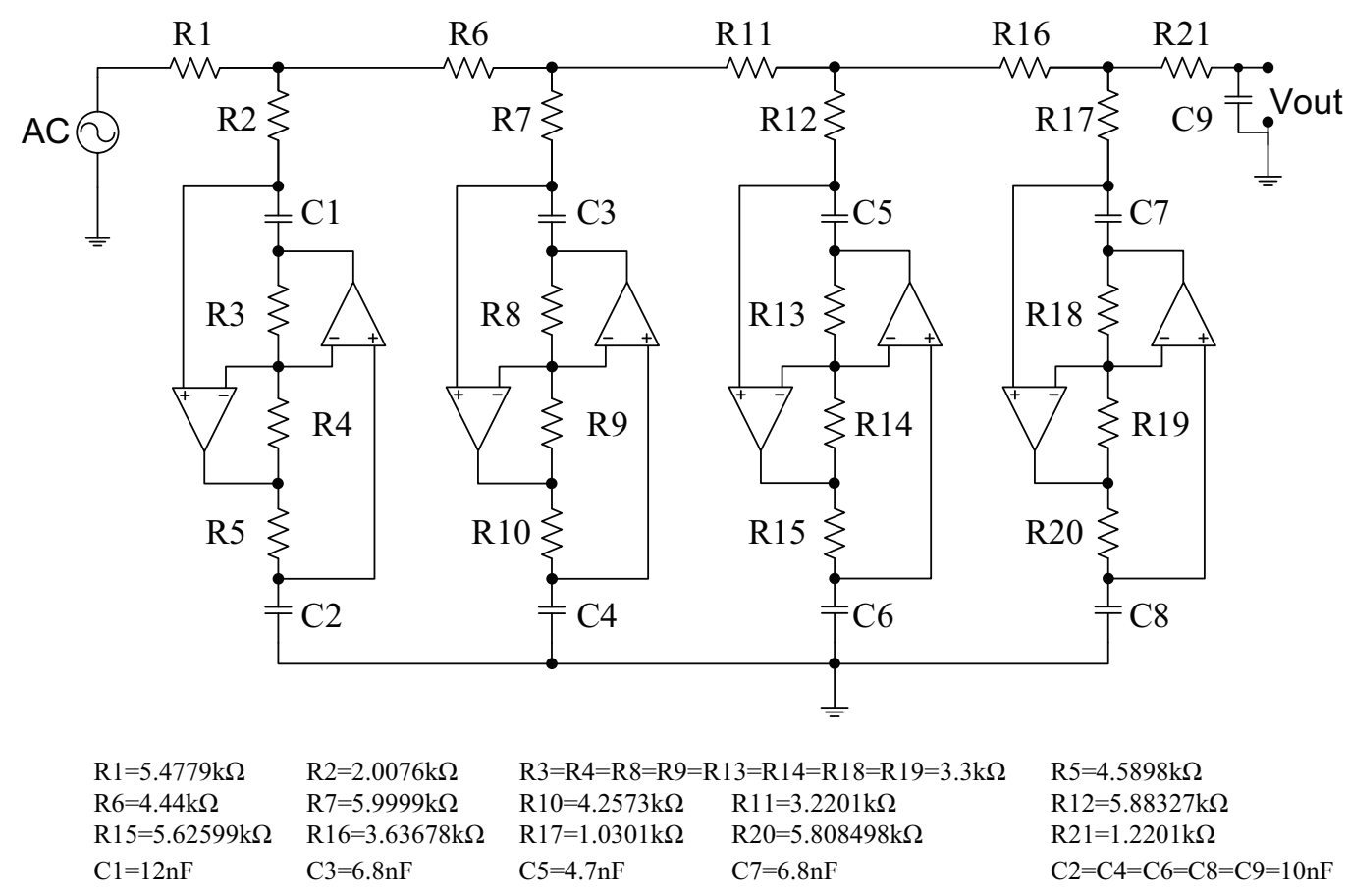

Figure 11: Schematic of an active low-pass filter.

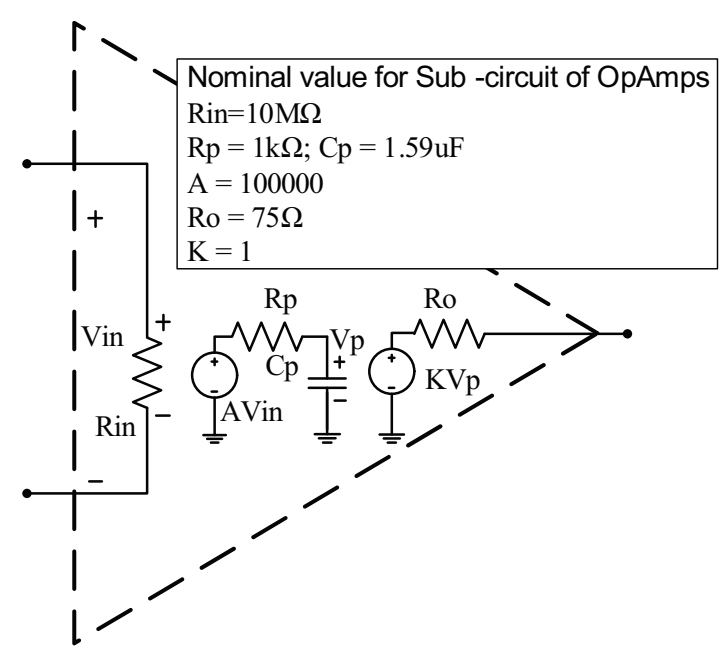

Figure 12: Op-Amp subcircuit model.

an independent normalized random variables, $\xi_{i}, i=1,2,3$. Three additional groups of parameters were represented with another three independent normalized Gaussian random variables, $\xi_{4}, \xi_{5}, \xi_{6}$. Those groups were, respectively, $\left\{C_{2}, C_{4}, C_{6}\right.$ and $\left.C_{8}\right\}$, \{all OpAmp internal resistors $\}$, and $\{$ all OpAmp internal capacitors $\}$. This problem 


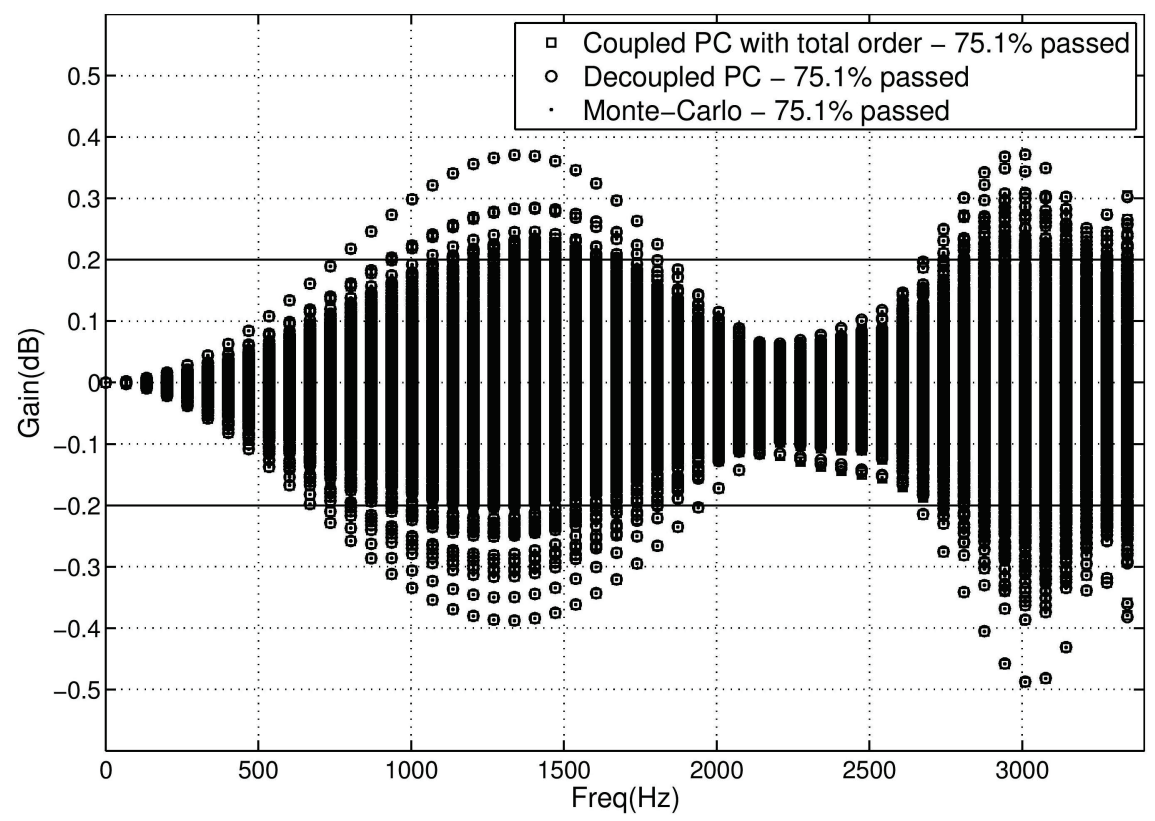

Figure 13: Pass-band response of the filter (Example 3).

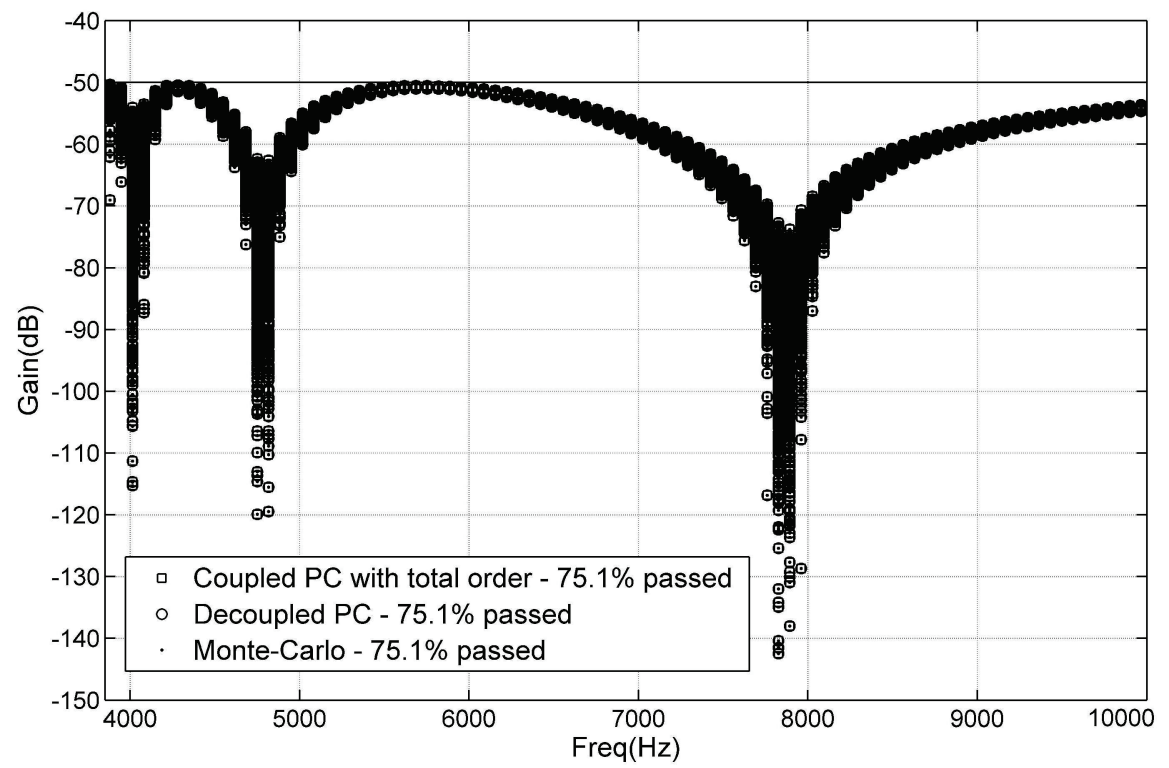

Figure 14: Stop-band response of the filter (Example 3).

configuration, therefore, created a $d$-dimensional random space with $d=6$. The relation between the uncertain circuit parameters and the normalized random variables 


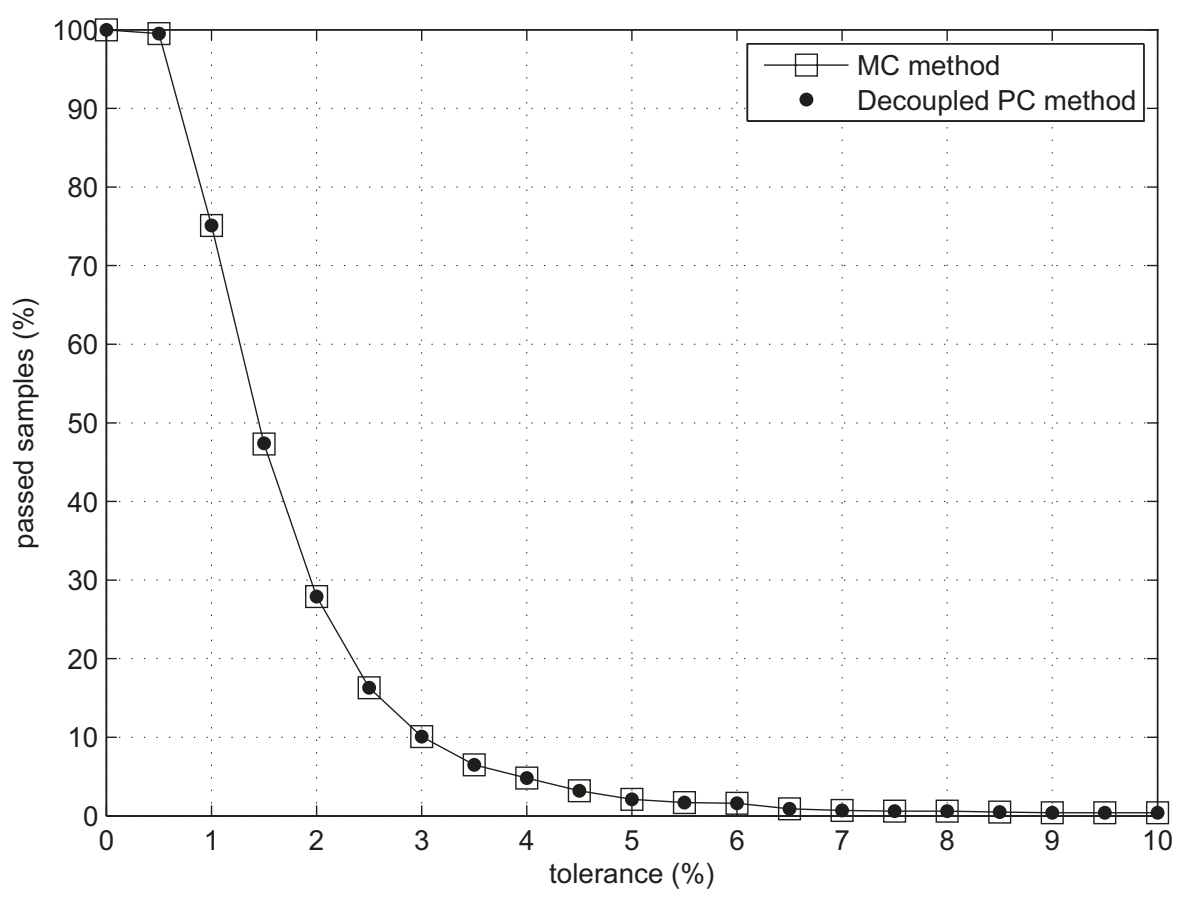

Figure 15: Yield of the Filter.

was represented by

$$
A_{i}=\bar{A}_{i}\left(1+\sigma \xi_{i}\right), \quad i=1, \cdots, 6
$$

where $A_{i}$ stands for the circuit parameter, $\bar{A}_{i}$ is its nominal value, $\xi_{i}$ is the normalized random variables (with Gaussian PDF, zero mean value and unit variance), and $\sigma$ represents the $\%$ tolerance on the circuit parameter. The nominal values for all the circuit parameters are shown in Figs. 11 and 12.

Figs. 13 and 14 show the simulation results, for gain-magnitude in the pass- and stop-band, respectively, obtained for 1000 samples of the circuit parameters, using the MC approach and standard PC method with fifth-order expansion $(M=5)$. Superimposed on these results are the results obtained from the proposed PC approach, for the same 1000 samples. The figures also report the $\%$ of samples that satisfied the above specifications using both the MC and proposed approach. As can be seen from those figures, the predicted number of samples satisfying the above specifications, obtained by MC and both standard and proposed decoupling PC approaches, 
is in excellent agreement. Fig. 15 plots the yield of the filter as the tolerance of the circuit components, $\sigma$, is changed from 0 to $10 \%$. It is worth noting that, while the MC method requires launching the simulation each time the tolerance of the circuit elements is changed, the PC method only needs updating the Hermite expansion of the output.

\subsubsection{The CPU Time Comparison}

This section provides the CPU time of three examples above using both the standard PC with total order and the proposed decoupled PC approaches. The computation of $\mathrm{CPU}$ time was done using $\mathrm{C}++$ at 300 frequency points.

As can be seen, the first row of Table 6 provides a CPU comparison of the analysis using the proposed decoupled PC approach whose $\boldsymbol{M}=\left[\begin{array}{lll}8 & 2 & 8\end{array}\right]^{T}$, and the standard coupled PC with third-order expansion $(M=3)$. As can be seen, a good speed-up is obtained using the proposed approach.

Table 6: Problems sizes and CPU computation time (CPU time for experiments that could not be completed due to lack of memory are marked with "-")

\begin{tabular}{|c|c|c|c|c|c|c|c|}
\hline \multirow{2}{*}{ Example } & \multicolumn{2}{|c|}{ Prob. Size } & \multicolumn{2}{|c|}{ Coupled PC } & \multicolumn{2}{|c|}{ Decoupled PC } & \multirow{2}{*}{ Speed-up } \\
\hline & $N$ & $d$ & $M$ & $\mathrm{CPU}(\mathrm{s})$ & $M^{T}$ & $\mathrm{CPU}(\mathrm{s})$ & \\
\hline Example 1 & 604 & 3 & 3 & 36.11 & {$\left[\begin{array}{lll}3 & 1 & 3\end{array}\right]$} & 1.05 & 34.39 \\
\hline Example 2 & 2416 & 4 & - & - & {$\left[\begin{array}{llll}8 & 2 & 8 & 5\end{array}\right]$} & 643.42 & - \\
\hline Example 3 & 112 & 6 & 5 & 1531.25 & {$\left[\begin{array}{llllll}1 & 2 & 1 & 5 & 1 & 1\end{array}\right]$} & 1.27 & 1205.71 \\
\hline
\end{tabular}

The second row of Table 6 provides the CPU time of the proposed decoupled

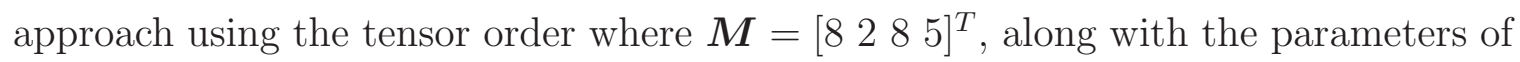
the problem size used in this example. In this example, the standard PC approach with total order could not be completed due to the large size of the problem.

The third row of Table 6 shows the CPU simulation time using the total-order PC approach and the proposed decoupled PC approach with tensor order of which 
$\boldsymbol{M}=\left[\begin{array}{llllll}1 & 2 & 1 & 5 & 1 & 1\end{array}\right]^{T}$, along with the other parameters pertaining to the problem size. As shown in this table, a speed-up to the tune of three orders of magnitude is obtained using the proposed approach. 


\section{Chapter 6}

\section{Application of Model Order Reduction to Variability Analysis}

Model-Order Reduction (MOR) has been used extensively as an effective approximation of many large scale mathematical models in different scientific and engineering fields, especially in circuit analysis and simulation. This advantage results from the fact that MOR enables reducing computational efforts in simulation process by approximating the complex high-order system with a much lower-order one, while still retaining the accuracy. For this reason, MOR could be a very useful technique to enhance the computational efficiency of the circuit variability analysis. Within this framework, there have been several approaches which were proposed in the literature to take this issue into consideration.

The perturbation-based techniques [22-24], basically apply MOR to the original stochastic system by representing the reduced models as an expansion of Taylor series in the random variables $\boldsymbol{\xi}$. Using these approaches, the reduced system is sampled at several values of $\boldsymbol{\xi}$, which are carefully chosen, and for each sampling point, an equation of the coefficients is constructed. Next, the system of these equations is solved to obtain the unknown coefficients. The disadvantage of the perturbational approaches comes from the requirement of applying MOR to every deterministic system at each 
sampling point. If the number of sampling points is huge and the original system is complex, these approaches could lead to the computational inefficiency.

In contrast to the perturbation-based approaches, the polynomial-chaos techniques could characterize the variability analysis of a system in the presence of process variations, hence, the application of MOR in this case only needs to be done once, to the deterministic augmented system, as described in $[2,25]$. However, one of the main drawbacks, which limits the attractiveness of MOR applications for variability analysis using the standard coupled PC approach, is the size of the augmented system, which is significantly bigger than the original system if a large number of random variables and a large number Hermite coefficients used for each random variable are required. For this reason, the $\mathrm{LU}$ factorization of the augmented system might not be done directly and needs to be solved using the iterative techniques [25]. In order to overcome this difficulty, another approach will be proposed in this chapter by applying MOR techniques on sub-systems, which are obtained after decoupling the augmented system using our decoupled PC method, instead of applying MOR to the augmented system directly. Due to the sparsity pattern, hence the size, of the subsystems similar to that of the original system of MNA equations, the computational cost for applying MOR to one sub-system is identical to the cost for applying MOR to an original deterministic system. This approach provides significant computational advantages over the PC methods without MOR.

In general, we could apply any existing MOR based on moment-matching techniques (MMTs) [26-34] to the systems arising from the PC-based variability analysis. MMTs can be generally classified into two categories [34]: explicit moment-matching approaches, such as AWE (Asymptotic Waveform Evaluation) [26,27] and implicit moment-matching approaches, such as Krylov subspace based techniques [30-33]. The AWE technique employs Padé rational approximations to explicitly match the moments. However, such an approach suffers from a significantly ill-conditioning 
problem and there will be no tangible improvement in the results whenever highorder approximations are attempted [34]. The indirect MMT, which belong to the second category mentioned above, could overcome this difficulty by matching the moments implicitly instead. Therefore, during the rest of this chapter, we will apply MOR to the circuit stochastic analysis by employing only the Krylov-Subspace-based projection techniques, which play a key role in the implicit MMT by constructing a reduced-model based on extracting leading eingenvalues of a given complex system.

This chapter is organized as follows. Section 6.1 will present an application of Krylov-subspace-based MOR method to the circuit stochastic analysis using the standard PC approach, while the proposed MOR approach which employs the decoupled PC algorithm will be described in details in Section 6.2. A numerical example will be presented in the last section to validate the accuracy as well as the computational advantage of the proposed approach.

\subsection{Applying MOR to Circuit Variability Analysis using Standard PC Approach}

This section describes the application of the Krylov-subspace projection techniques to obtain a reduced model for the augmented system arising from the coupled PC approach. Without loss of generality, only the augmented system using the total order will be taken into account, since the size of this augmented system is generally smaller than the size of the one with the tensor order, which means less computational effort required.

Let us start by recalling the augmented system of DAEs derived in (82), using the standard PC approach with total lexicographic order, which is given by:

$$
\left(\overline{\boldsymbol{G}}^{*}+s \overline{\boldsymbol{C}}^{*}\right) \overline{\boldsymbol{X}}^{*}(s)=\overline{\boldsymbol{U}}^{*}
$$


where the superscript " *" is used to distinguish the total lexicographic order from the tensor order. Multiplying both sides of (118) by $-\bar{G}^{*-1}$ and rearranging this system of equations yields the following:

$$
s \boldsymbol{A}^{*} \overline{\boldsymbol{X}}^{*}(s)=\overline{\boldsymbol{X}}^{*}(s)-\boldsymbol{R}^{*}
$$

where $\boldsymbol{A}^{*}=-\overline{\boldsymbol{G}}^{*-1} \overline{\boldsymbol{C}}^{*}$ and $\boldsymbol{R}^{*}=\overline{\boldsymbol{G}}^{*-1} \overline{\boldsymbol{U}}^{*}$.

From (119), the system response $\overline{\boldsymbol{X}}^{*}(s)$ can be written as follows:

$$
\overline{\boldsymbol{X}}^{*}(s)=\left(\boldsymbol{I}-s \boldsymbol{A}^{*}\right)^{-1} \boldsymbol{R}^{*}
$$

Expanding (120) using the Taylor series will provide us the formulas to calculate the moments of the system response, as shown in the following:

$$
\overline{\boldsymbol{X}}^{*}(s)=\left(\boldsymbol{I}+s \boldsymbol{A}^{*}+s^{2} \boldsymbol{A}^{* 2}+\cdots\right) \boldsymbol{R}^{*}=\sum_{q=0}^{\infty} s^{q} \boldsymbol{M}^{*}{ }_{q}
$$

where $\boldsymbol{M}_{q}^{*}=\boldsymbol{A}^{* q} \boldsymbol{R}^{*}$ is the vector of moments with order $q$ (for $\left.q=1,2, \cdots\right)$.

Next, define a Krylov subspace as:

$$
\begin{aligned}
\kappa\left(\boldsymbol{A}^{*}, \boldsymbol{R}^{*}, k\right)=\text { ColumnSpace }\left\{\boldsymbol{R}^{*}, \boldsymbol{A}^{*} \boldsymbol{R}^{*}, \boldsymbol{A}^{* 2} \boldsymbol{R}^{*}, \cdots, \boldsymbol{A}^{* k-1} \boldsymbol{R}^{*}\right\} \\
=\text { ColumnSpace }\left\{\boldsymbol{Q}^{*}\right\}
\end{aligned}
$$

where the matrix $\boldsymbol{Q}^{*}$ is the orthogonal basis of the Krylov subspace $\kappa\left(\boldsymbol{A}^{*}, \boldsymbol{R}^{*}, k\right)$, which spans k columns of $\boldsymbol{Q}^{*}$, and given by:

$$
\boldsymbol{Q}^{*}=\left[\begin{array}{llll}
\boldsymbol{q}_{1} & \boldsymbol{q}_{2} & \cdots & \boldsymbol{q}_{k}
\end{array}\right]
$$

where $\boldsymbol{q}_{i}$ (for $\left.i=1,2, \cdots, k\right)$ is the $i^{t h}$ column of matrix $\boldsymbol{Q}^{*}\left(\boldsymbol{q}_{i}^{T} \boldsymbol{q}_{j}=1\right.$ if $i=j$ and 
$\boldsymbol{q}_{i}^{T} \boldsymbol{q}_{j}=0$ if $\left.i \neq j\right)$. This matrix then will satisfy the following property of the Krylov subspace:

$$
A^{*} Q^{*}=Q^{*} H^{*}
$$

where $\boldsymbol{H}^{*}$ is the so-called upper-Hessenberg Matrix whose $\boldsymbol{H}^{*}{ }_{i j}=0$ for $i>j+1$.

The equation in (124) could be used to recursively calculate the orthogonal basis $\boldsymbol{Q}^{*}$ using Arnoldi algorithm [31] as described in the following pseudo-code.

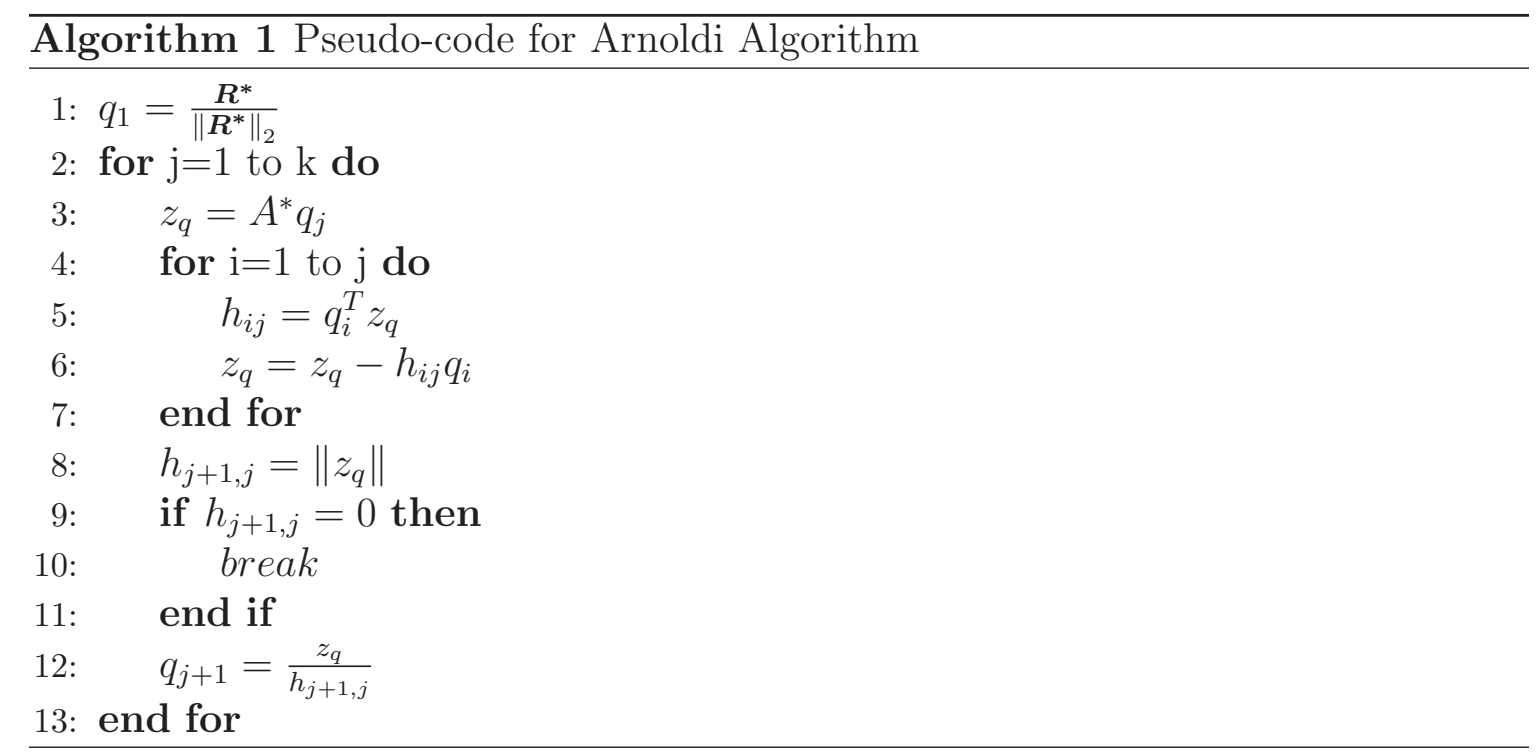

By a change of variables using the known matrix $Q^{*}$ above, the vector $\overline{\boldsymbol{X}}^{*}(s)$ could be mapped into a much smaller vector $\check{\boldsymbol{X}}^{*}(s)$ as follows:

$$
\overline{\boldsymbol{X}}^{*}(s)=\boldsymbol{Q}^{*} \check{\boldsymbol{X}}^{*}(s)
$$

Substituting (125) into (119) and premultiplying both sides by $\boldsymbol{Q}^{* T}$ generates the following system of equations:

$$
s \boldsymbol{Q}^{* T} \boldsymbol{A}^{*} \boldsymbol{Q}^{*} \check{\boldsymbol{X}}^{*}(s)=\check{\boldsymbol{X}^{*}}(s)-\boldsymbol{Q}^{* T} \boldsymbol{R}^{*}
$$


Rearranging (126), the frequency response of the reduced system can be written as:

$$
\check{\boldsymbol{X}}^{*}(s)=\left(\boldsymbol{I}-s \boldsymbol{H}^{*}\right)^{-1} \boldsymbol{Q}^{* T} \boldsymbol{R}^{*}
$$

where $\boldsymbol{H}^{*}=\boldsymbol{Q}^{* T} \boldsymbol{A}^{*} \boldsymbol{Q}^{*}$, which is obtained from (124).

The frequency response of the reduced system in (127) is a good approximation of the augmented system response in (120) and this reduced system of order $k$ is proved to preserve the first $k$ moments of the original augmented system [33]. Therefore, using this approximation allows us to implicitly match the moments of the augmented system response, thereby avoiding the numerical ill-conditioned problem as in the direct moment-matching like AWE.

However, as can be seen in the Krylov-subspace-based approach above, one of the most costly processes is to find $\boldsymbol{A}^{*}$ by solving the following equation:

$$
\bar{G}^{*} A^{*}=-\bar{C}^{*}
$$

The equation (128) requires the LU factorization of the augmented matrix $\overline{\boldsymbol{G}}^{*}$ which indeed is huge due to the size of the augmented system. Therefore, this process might be difficult, or even impossible, to be done explicitly and needs to be solved using iterative techniques, as described in [25]. Nevertheless, the computational cost of this MOR approach, even when using iterative methods, is still high if the stochastic analysis involves many random variables and requires a large number of Hermite orders.

The proposed MOR approach, which will be presented in the next section, could overcome these problems, providing its computational efficiency. 


\subsection{Applying MOR to Circuit Variability Analysis using Decoupled PC Approach}

In this section, the application of MOR to the circuit stochastic analysis arising from the proposed PC techniques is presented. Particularly, the systems to which we target to apply MOR are those obtained by decoupling the augmented system in (95). It should be noted that the sub-matrices of these sub-systems have the same size $\mathrm{N}$ and sparsity pattern with the corresponding matrices of the original MNA, therefore, it is possible to apply the same MOR techniques as for the normal circuit system to these sub-systems.

Let us consider first the augmented decoupling system in (108), which could be rewritten as follows:

$$
\left(\sum_{\boldsymbol{\beta}} \boldsymbol{F}_{\boldsymbol{\beta}}(\boldsymbol{\lambda}) \otimes \hat{\boldsymbol{G}}_{\boldsymbol{\beta}}+s \sum_{\boldsymbol{\beta}} \boldsymbol{F}_{\boldsymbol{\beta}}(\boldsymbol{\lambda}) \otimes \hat{\boldsymbol{C}}_{\boldsymbol{\beta}}\right)\left(\overline{\boldsymbol{\psi}}^{\top} \overline{\boldsymbol{\Gamma}}\right)^{-1} \tilde{\boldsymbol{Y}}(s)=\overline{\boldsymbol{\Gamma}}^{-1} \overline{\boldsymbol{U}}
$$

Performing the changes of variables:

$$
\tilde{\boldsymbol{Z}}(s)=\left(\overline{\boldsymbol{\psi}}^{\top} \overline{\boldsymbol{\Gamma}}\right)^{-1} \tilde{\boldsymbol{Y}}(s)
$$

leads us to the following system of equations:

$$
\left(\sum_{\boldsymbol{\beta}} \boldsymbol{F}_{\boldsymbol{\beta}}(\boldsymbol{\lambda}) \otimes \hat{\boldsymbol{G}}_{\boldsymbol{\beta}}+s \sum_{\boldsymbol{\beta}} \boldsymbol{F}_{\boldsymbol{\beta}}(\boldsymbol{\lambda}) \otimes \hat{\boldsymbol{C}}_{\boldsymbol{\beta}}\right) \tilde{\boldsymbol{Z}}(s)=\overline{\boldsymbol{\Gamma}}^{-1} \overline{\boldsymbol{U}}
$$


Let us define $\tilde{\boldsymbol{G}}, \tilde{\boldsymbol{C}}$ and $\tilde{\boldsymbol{U}}(s)$ so that:

$$
\begin{array}{r}
\tilde{\boldsymbol{G}}=\sum_{\boldsymbol{\beta}} \boldsymbol{F}_{\boldsymbol{\beta}}(\boldsymbol{\lambda}) \otimes \hat{\boldsymbol{G}}_{\boldsymbol{\beta}} \\
\tilde{\boldsymbol{C}}=\sum_{\boldsymbol{\beta}} \boldsymbol{F}_{\boldsymbol{\beta}}(\boldsymbol{\lambda}) \otimes \hat{\boldsymbol{C}}_{\boldsymbol{\beta}} \\
\tilde{\boldsymbol{U}}=\overline{\boldsymbol{\Gamma}}^{-1} \overline{\boldsymbol{U}}
\end{array}
$$

As we explained before in Chapter $5.2, \tilde{\boldsymbol{G}}$ and $\tilde{\boldsymbol{C}}$ can be shown to be block-diagonal as follows:

$$
\begin{gathered}
\tilde{G}=\left[\begin{array}{cccc}
G_{0} & 0 & \cdots & 0 \\
0 & G_{1} & \cdots & 0 \\
\vdots & 0 & \ddots & \vdots \\
\vdots & 0 & 0 & G_{|\alpha|_{\max }}
\end{array}\right] \\
\tilde{\boldsymbol{C}}=\left[\begin{array}{cccc}
\boldsymbol{C}_{0} & 0 & \cdots & 0 \\
0 & C_{1} & \cdots & 0 \\
\vdots & 0 & \ddots & \vdots \\
\vdots & 0 & 0 & \boldsymbol{C}_{|\boldsymbol{\alpha}|_{\max }}
\end{array}\right]
\end{gathered}
$$

where $\boldsymbol{G}_{i}, \boldsymbol{C}_{i} \in \mathbb{R}^{N \times N}$ are the $i^{\text {th }}$ block-matrices on the diagonal of $\tilde{\boldsymbol{G}}$ and $\tilde{\boldsymbol{C}}$, respectively ( $i$ is the rank of the multi-index and $i=1,2, \cdots,|\boldsymbol{\alpha}|_{\max }$ ). $|\boldsymbol{\alpha}|_{\max }$ is the highest rank of the multi-indices $\boldsymbol{\alpha}$. 
Moreover, $\tilde{\boldsymbol{Z}}(s)$ and $\tilde{\boldsymbol{U}}$ can be expressed as

$$
\tilde{\boldsymbol{Z}}=\left[\begin{array}{c}
\boldsymbol{Z}_{0} \\
\boldsymbol{Z}_{1} \\
\vdots \\
\boldsymbol{Z}_{|\boldsymbol{\alpha}|_{\max }}
\end{array}\right] \tilde{\boldsymbol{U}}=\left[\begin{array}{c}
\boldsymbol{U}_{0} \\
\boldsymbol{U}_{1} \\
\vdots \\
\boldsymbol{U}_{|\boldsymbol{\alpha}|_{\max }}
\end{array}\right]
$$

where $\boldsymbol{Z}_{i} \in \mathbb{C}^{N}$ and $\boldsymbol{U}_{i} \in \mathbb{C}^{N}$ are the $i^{\text {th }}$ block-vectors of $\tilde{\boldsymbol{Z}}$ and $\tilde{\boldsymbol{U}}$, respectively.

Therefore, the sub-systems obtained after decoupling (131) are given by

$$
\left(\boldsymbol{G}_{i}+{ }_{s} \boldsymbol{C}_{i}\right) \boldsymbol{Z}_{i}(s)=\boldsymbol{U}_{i}, i=1,2, \cdots,|\boldsymbol{\alpha}|_{\max }
$$

MOR techniques can now be applied to the sub-systems in (138) to approximate the responses $\boldsymbol{Z}_{i}(s)$. Particularly, consider the $i^{\text {th }}$ sub-system, multiplying both sides of (138) by $-\boldsymbol{G}_{i}^{-1}$ and rearranging it yields the following response of the sub-system:

$$
\boldsymbol{Z}_{i}(s)=\left(\boldsymbol{I}-s \boldsymbol{A}_{i}\right)^{-1} \boldsymbol{R}_{i}
$$

where $\boldsymbol{A}_{i}=-\boldsymbol{G}_{i}^{-1} \boldsymbol{C}_{i}$ and $\boldsymbol{R}_{i}=\boldsymbol{G}_{i}^{-1} \boldsymbol{U}_{i}$.

Similar to the previous section, expanding (139) in terms of Taylor series provides us the formulas for the moments of $\boldsymbol{Z}_{i}(s)$, which is $\boldsymbol{M}_{i}^{q}=\boldsymbol{A}_{i}^{q} \boldsymbol{R}_{i}($ for $q=1,2, \cdots)$.

Assuming that $\boldsymbol{Q}_{i}$ is the orthogonal basis of the Krylov subspace $\kappa^{i}\left(\boldsymbol{A}_{i}, \boldsymbol{R}_{i}, m\right)$, which spans $m$ columns of $\boldsymbol{Q}_{i}$, given by:

$$
\begin{aligned}
\kappa^{i}\left(\boldsymbol{A}_{i}, \boldsymbol{R}_{i}, m\right)=\text { ColumnSpace }\left\{\boldsymbol{R}_{i}, \boldsymbol{A}_{i} \boldsymbol{R}_{i}, \boldsymbol{A}_{i}^{2} \boldsymbol{R}_{i}, \cdots,\right. & \left.\boldsymbol{A}_{i}^{m-1} \boldsymbol{R}_{i}\right\} \\
& =\text { ColumnSpace }\left\{\boldsymbol{Q}_{i}\right\}
\end{aligned}
$$


where,

$$
\boldsymbol{Q}_{i}=\left[\begin{array}{llll}
\boldsymbol{q}_{i}^{1} & \boldsymbol{q}_{i}^{2} & \cdots & \boldsymbol{q}_{i}^{m}
\end{array}\right]
$$

By a change or variables using the matrix $\boldsymbol{Q}_{i}$, which could be obtained by applying the Arnoldi algorithm described in the previous section to the Krylov subspace $\kappa^{i}\left(\boldsymbol{A}_{i}, \boldsymbol{R}_{i}, m\right)$, the vector $\boldsymbol{Z}_{i}(s)$ could be mapped into a smaller vector $\check{\boldsymbol{Z}}_{i}(s)$ as follows:

$$
\check{Z}_{i}(s)=\boldsymbol{Q}_{i}^{-1} \boldsymbol{Z}_{i}(s)
$$

Using (142), the systems of equation in (139) can be rewritten as:

$$
s \boldsymbol{A}_{i} \boldsymbol{Q}_{i} \check{Z}_{i}(s)=\boldsymbol{Q}_{i} \check{\boldsymbol{Z}}_{i}(s)-\boldsymbol{R}_{i}
$$

Premultiplying both sides of (143) by $\boldsymbol{Q}_{i}^{T}$ generates the following system of equations:

$$
s \boldsymbol{Q}_{i}^{T} \boldsymbol{A}_{i} \boldsymbol{Q}_{i} \check{Z}_{i}(s)=\check{\boldsymbol{Z}}_{i}(s)-\boldsymbol{Q}_{i}^{T} \boldsymbol{R}_{i}
$$

Rearranging (144) allows us to find the response of the reduced system as:

$$
\check{\boldsymbol{Z}}_{i}(s)=\left(\boldsymbol{I}-s \boldsymbol{H}_{i}\right)^{-1} \boldsymbol{Q}_{i}^{T} \boldsymbol{R}_{i}
$$

where $\boldsymbol{H}_{i}=\boldsymbol{Q}_{i}^{T} \boldsymbol{A}_{i} \boldsymbol{Q}_{i}$ is the upper-Hessenberg matrix.

Using (145) to find the approximations of all sub-system responses (sweeping $i$ from 0 to $|\boldsymbol{\alpha}|_{\max }$ ) and performing the change of variable, as in (130), will provide us a good approximation of the original augmented system response.

Furthermore, it can be seen easily that one of the major computational costs in this approach is to find $\boldsymbol{A}_{i}$ by solving the following equation:

$$
G_{i} A_{i}=-C_{i}
$$


The equation (146) requires the LU factorization of each sub-matrix $\boldsymbol{G}_{i}$, for $i=$ $1,2, \cdots,|\boldsymbol{\alpha}|_{\max }$. However, this process only needs to be done once for each subsystem which has the same size and sparsity pattern of the original circuit system, so it does not take much computational effort to be completed. The significant advantage of this approach is that, by applying MOR to the sub-systems, we do not have to cope with the difficulty of direct LU factorization, as described in Section 6.1.

\subsection{Numerical Example}

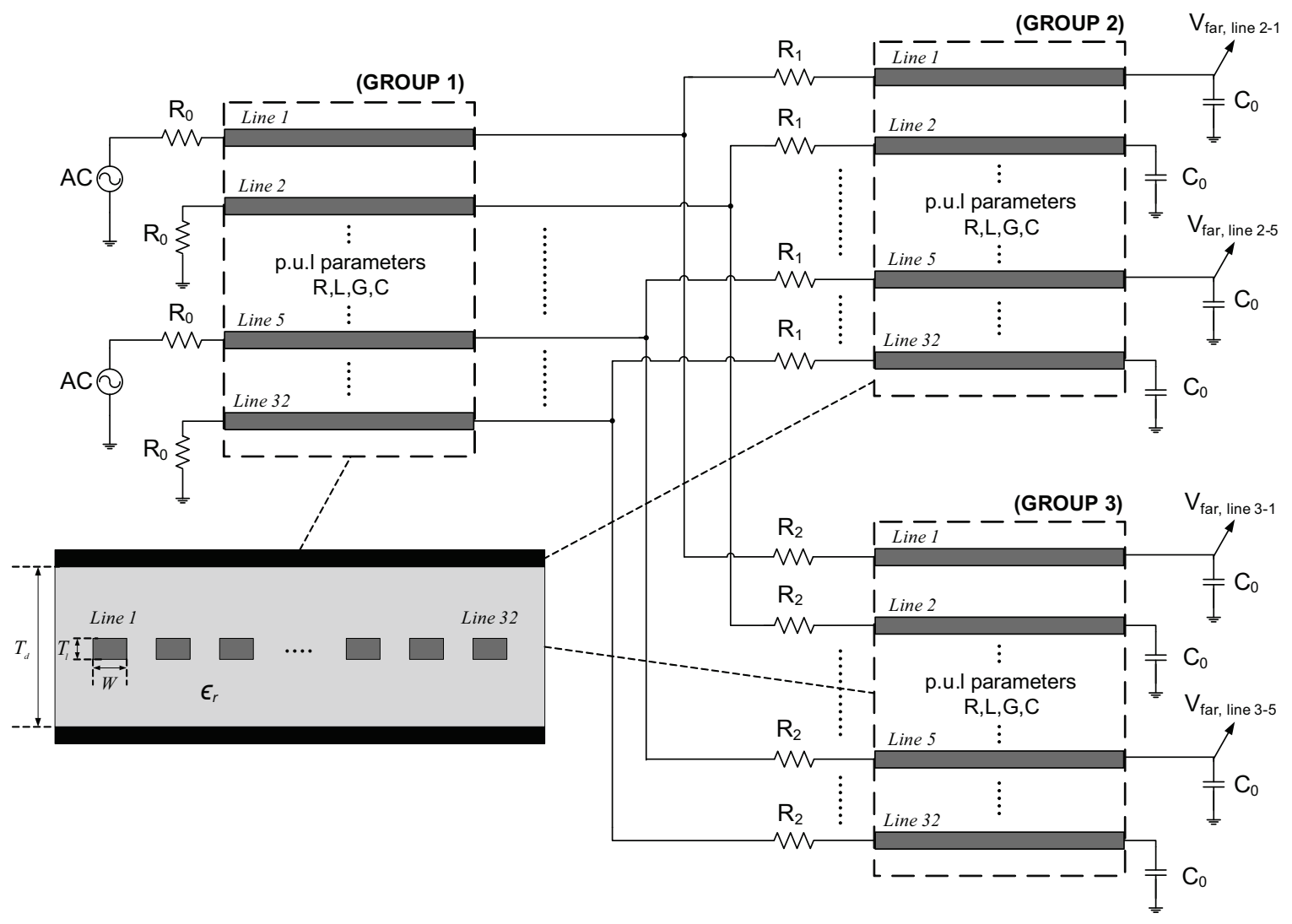

Figure 16: Circuit of three 32-MTL groups and the cross-section of each MTL group (bottom left of the figure)

In this example, the proposed algorithm is implemented to characterize the variability of the circuit which comprises three groups of multi-conductor transmission 
line (MTL), with 32 TLs for each group, as shown in Fig. 16. Each TL has a length of $10 \mathrm{~cm}$ and its macromodel is constructed using 50 sections of lumped RLGC segmentation. The first of every four lines of MTL group one is excited by an AC voltage source with $1 \mathrm{~V}$ amplitude at its near-end while the remaining lines are kept quiet.

The sources of uncertainty in this circuit are assumed to arise from four groups of parameters which were represented with four independent normalized Gaussian random variables (with zero mean and unit variance), $\xi_{1}, \xi_{2}, \xi_{3}, \xi_{4}$. Those groups were, respectively, $\left\{\right.$ all resistors $\left.R_{0}\right\},\left\{\right.$ all resistors $\left.R_{1}\right\},\left\{\right.$ all resistors $\left.R_{2}\right\}$ and $\left\{\right.$ all resistors $\left.C_{0}\right\}$. This problem configuration, therefore, created a $d$-dimensional random space with $d=4$.

The relation between the uncertain circuit parameters and the normalized random variables was represented by

$$
\begin{aligned}
& R_{0}=\bar{R}_{0}\left(1+\sigma \xi_{1}\right) \\
& R_{1}=\bar{R}_{1}\left(1+\sigma \xi_{2}\right) \\
& R_{2}=\bar{R}_{2}\left(1+\sigma \xi_{3}\right) \\
& C_{0}=\bar{C}_{0}\left(1+\sigma \xi_{4}\right)
\end{aligned}
$$

where $\sigma=0.1$ is the normalized standard deviation, while $\bar{R}_{0}, \bar{R}_{1}, \bar{R}_{2}$ and $\bar{C}_{0}$ are the nominal values of the parameters, given by, $\bar{R}_{0}=50 \Omega, \bar{R}_{1}=10 \Omega, \bar{R}_{2}=20 \Omega$ and $\bar{C}_{0}=1 p F$.

Figs. 17-20 show the mean value and the $\pm 3 \sigma$ tolerances of the magnitude of far-end voltages of four active lines, 1,9,17 and 25 of MTL group two, which are obtained by Monte-Carlo method using HSPICE ${ }^{\circledR}$. Superimposed on these results are the results obtained from both standard and proposed PC approaches where we applied the MOR based on Krylov-subspace projection techniques as described in previous sections of this chapter. Those figures demonstrate a good agreement 


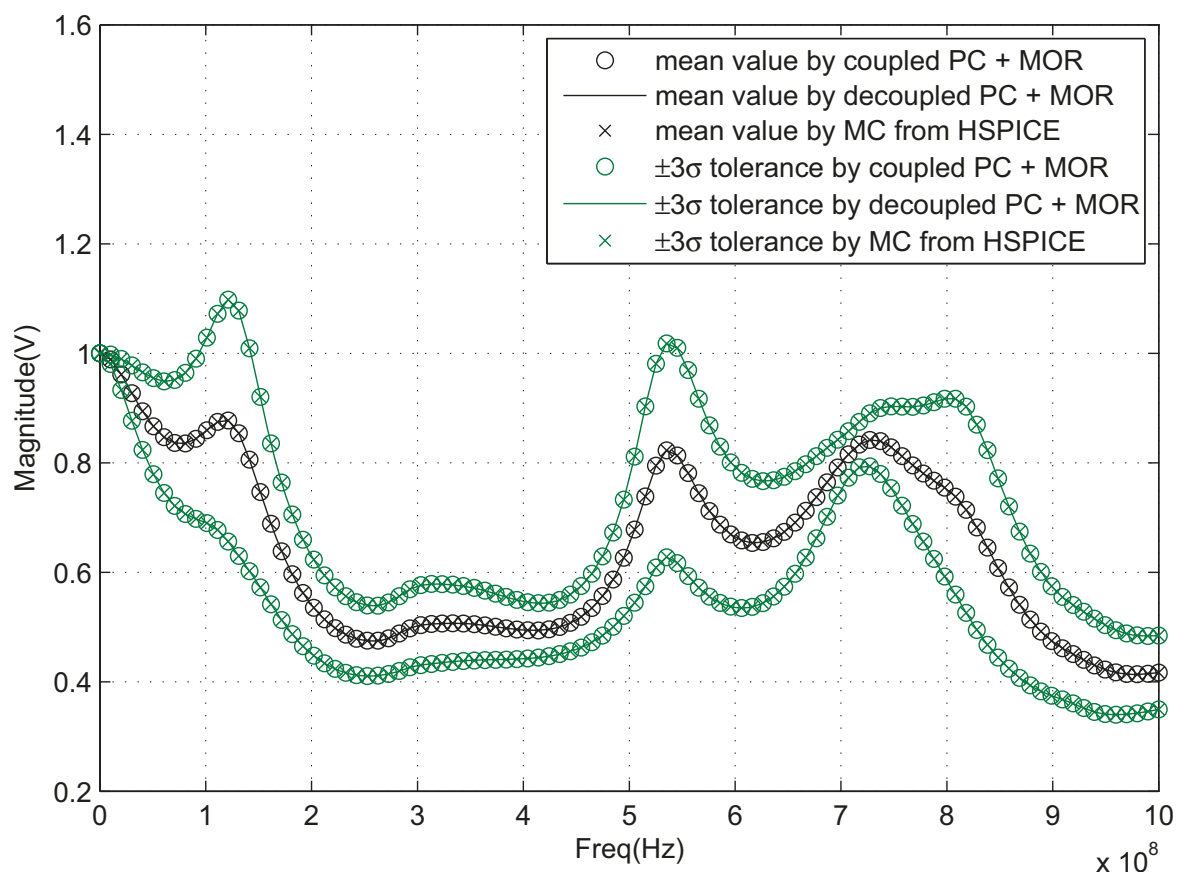

Figure 17: Statistical analysis for the magnitude of the far-end voltage of line 2-1.

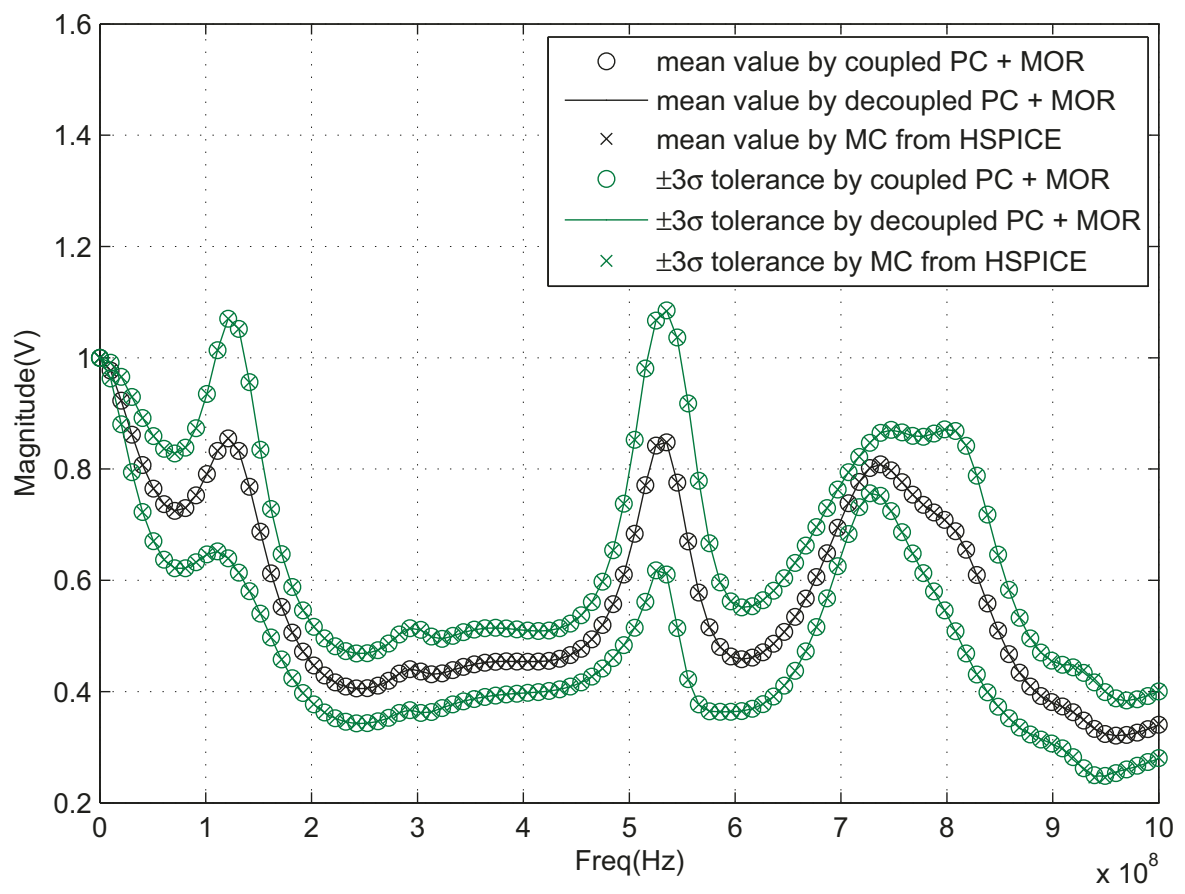

Figure 18: Statistical analysis for the magnitude of the far-end voltage of line 2-9.

between the PC approaches (with MOR) and the classical MC approach.

Table 7 provides the CPU time for this example using the PC approaches, with 


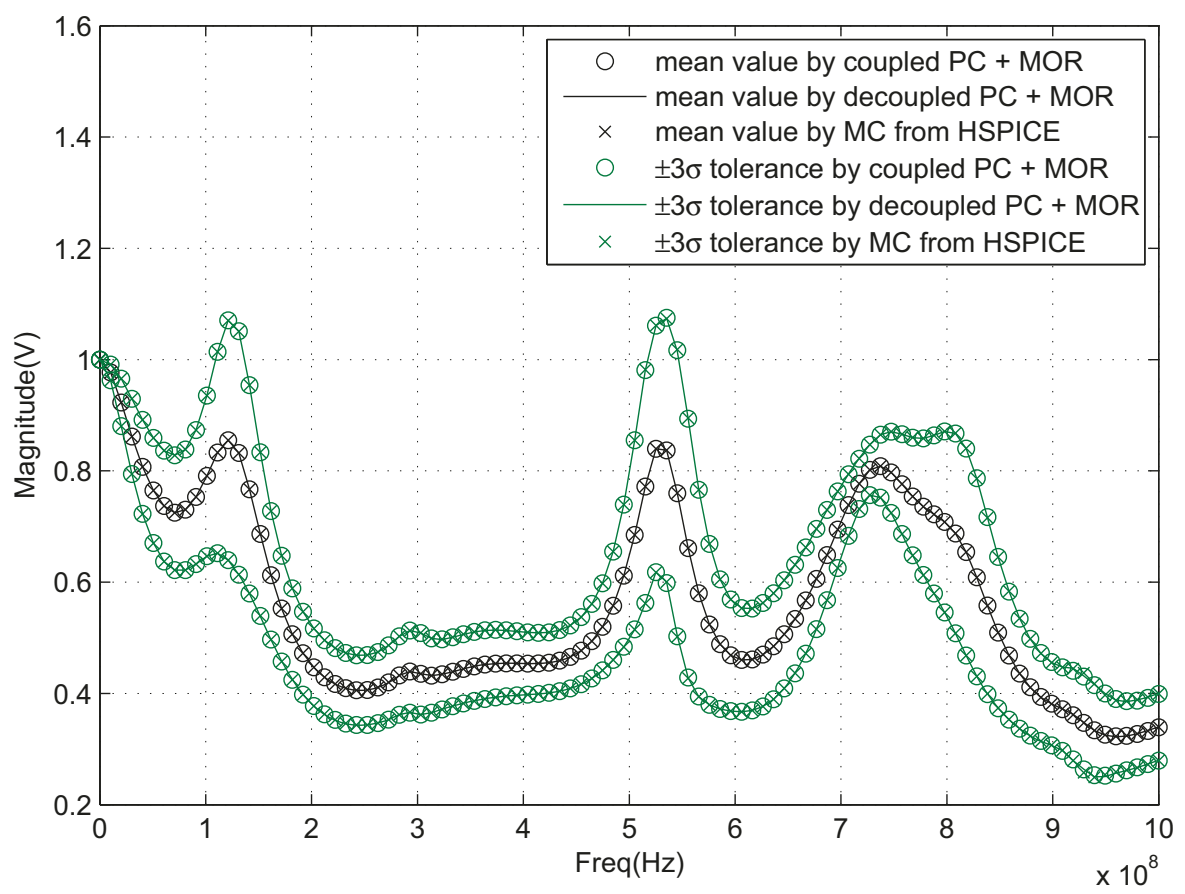

Figure 19: Statistical analysis for the magnitude of the far-end voltage of line 2-17.

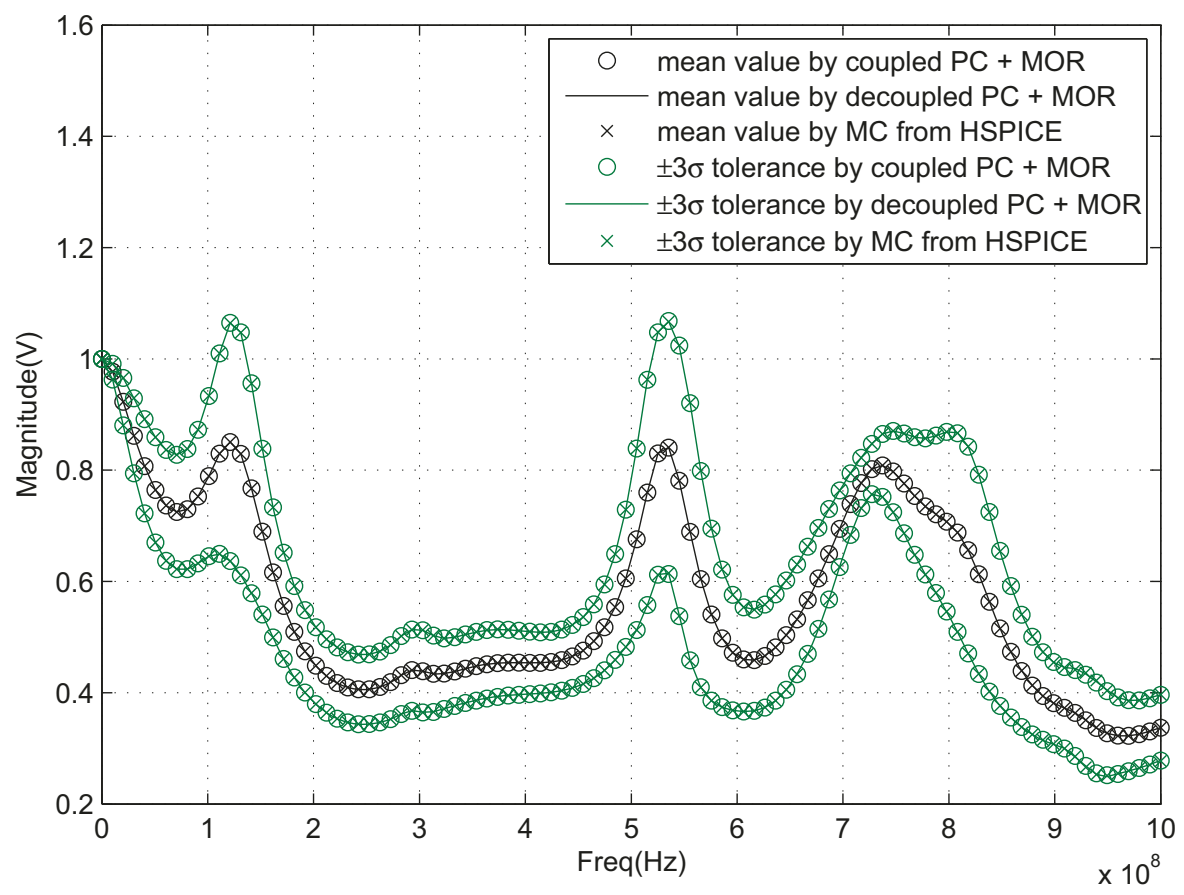

Figure 20: Statistical analysis for the magnitude of the far-end voltage of line 2-25. 
and without the application of MOR. The computation of CPU time was done using a $\mathrm{C}++$ implementation of these approaches and evaluating at 100 frequency points. It should also be noted that the coupled PC here is the standard one which uses the total order. As can be seen, compared to the case of standard coupled PC without MOR, applying MOR directly to the augmented system (arising from coupled PC with second-order expansion) gains a speed-up of 23.24 times while a better speedup, which is 27.61 times faster, could be obtained using our proposed MOR approach, where $\boldsymbol{M}=\left[\begin{array}{llll}2 & 1 & 1 & 2\end{array}\right]^{T}$. In addition, this table also shows that without MOR, the decoupled PC could obtain a speed-up of more than 5 times over the standard coupled PC. It should be noted that the CPU time of the decoupled PC approaches could be significantly reduced if their implementation is done in parallel on a suitable multicore platform.

Table 7: CPU computation time with and without MOR (speed-ups are obtained compared with the case of coupled PC without MOR)

\begin{tabular}{c|c|c|c|c}
\hline Method & System Size & No. of Systems & CPU(seconds) & Speed-up \\
\hline \hline Coupled PC (total order) & 219120 & 1 & 1325.69 & - \\
\hline Decoupled PC & 14608 & 36 & 228.07 & 5.81 \\
\hline Coupled PC + MOR & 700 & 1 & 57.04 & 23.24 \\
\hline Decoupled PC + MOR & 200 & 36 & 48.01 & 27.61 \\
\hline
\end{tabular}




\section{Chapter 7}

\section{Conclusions and Future Work}

\subsection{Conclusions}

One of the major constraints of variability analysis based on the Polynomial Chaos techniques comes from the size of the augmented system, which grows significantly with the increasing number of random variables and the number of Hermite coefficients used with each random variable. This thesis presented a novel approach to overcome the above difficulty by decoupling the system of equations, arising from the Hermite-based Polynomial Chaos approach for variability analysis, into smaller subsystems. The thesis also presented a new theoretical result to characterize the structure of the augmented matrices and show its proximity to a different structure that lends itself more naturally to decoupling.

Additionally, the application of the model order reduction techniques to the subsystems, obtained after decoupling the augmented system arising from the proposed PC algorithm, is also presented in the thesis.

Numerical examples have been presented to validate the proposed technique, which shows the significant computational advantage by comparing the results obtained from the standard coupled, and the proposed decoupled PC approach along with Monte Carlo simulations. 


\subsection{Future Work}

Several directions for further research based on the results of this thesis are described below:

- Parallel implementation: It should be noted, the major advantage of the proposed algorithm is that, the matrices of decoupled sub-systems could be factorized independently, and hence, this could be done in parallel on a suitable multi-core platform. It could be easily seen that the parallel implementation of our decoupling techniques will obtain a much greater speed-up, especially if we employ our proposed MOR application.

- Improvement of the decoupling approach: This could be done by a further scrutiny in the structure of the augmented system arising from our proposed approach. For example, more investigation could be done on the structure of the constant matrices $\boldsymbol{K}_{i}$ to find their approximated structures with better proximity relation. In addition, considering the the block matrices $\boldsymbol{L}_{k}$ as shown in (94), it could be easily seen that the source of the difference between these matrices only arises from $\lambda_{k}$, which is the $k^{\text {th }}$ eigenvalue of the matrix $\boldsymbol{K}_{\mathbf{1}}=$ $\boldsymbol{A}_{\boldsymbol{L}}+\boldsymbol{A}_{U}$. It is interesting to find that there is a relationship between the eigenvalues of $\boldsymbol{K}_{\mathbf{1}}$ which could be grouped into a vector $\Delta$ as follows:

$$
\Delta^{T}=\left\{\begin{array}{cccccc}
{\left[\begin{array}{ccccccc}
-\lambda_{p} & \cdots & -\lambda_{1} & \lambda_{1} & \cdots & \lambda_{p}
\end{array}\right]} & \mathrm{M}=\text { odd number, } p=\frac{M+1}{2} \\
{\left[\begin{array}{lllllll}
-\lambda_{p} & \cdots & -\lambda_{1} & 0 & \lambda_{1} & \cdots & \lambda_{p}
\end{array}\right]} & \mathrm{M}=\text { even number, } p=\frac{M}{2}
\end{array}\right.
$$

As can be seen, (151) shows us that any eigenvalue of $\boldsymbol{K}_{\mathbf{1}}$, except zero eigenvalue, will have the same absolute value with another eigenvalue of $\boldsymbol{K}_{\mathbf{1}}$, in an opposite direction. This discovery could be used to investigate the similarity between 
the sub-systems, and possibly, reduce the number of sub-systems which needs to be solved.

- Time-domain formulation: In this thesis, the proposed decoupled PC method has just been performed in frequency domain, however, this approach could be extended to the time domain analysis with nonlinear elements, which is expected to yield significant computational advantages over the standard PC approach as well as the Monte-Carlo method.

- Sensitivity analysis: As being explained in the thesis, the proposed PC approach was developed using the tensor order, which allows us to choose different expansion orders for different random variables. Due to the fact that the effects of random variables on the circuit response are normally not equal, the flexibility of the tensor order could enable a future research in sensitivity analysis basing on the proposed decoupled algorithm.

- Circuit optimization: Development of a circuit optimization engine which uses the proposed stochastic based analysis could be considered as another candidate of research interest. 


\section{List of References}

[1] D. Xiu and G. E. Karniadakis, "The wiener-askey polynomial chaos for stochastic differential equations," SIAM J. Sci. Comput., vol. 24, no. 2, pp. 619-644, Feb. 2002. [Online]. Available: http://dx.doi.org/10.1137/S1064827501387826

[2] S. Vrudhula, J. Wang, and P. Ghanta, "Hermite polynomial based interconnect analysis in the presence of process variations," IEEE Trans. Computer-Aided Design of Integrated Circ. Sys., vol. 25, no. 10, pp. 2001 -2011, Oct. 2006.

[3] I. Stievano, P. Manfredi, and F. Canavero, "Stochastic analysis of multiconductor cables and interconnects," IEEE Trans. Electromagn. Compat., vol. 53, no. 2, pp. $501-507$, May 2011.

[4] D. Spina, F. Ferranti, T. Dhaene, L. Knockaert, G. Antonini, and D. Vande Ginste, "Variability analysis of multiport systems via polynomial-chaos expansion," IEEE Trans. Microw. Theory Techn., vol. 60, no. 8, pp. 2329 -2338, Aug. 2012.

[5] I. Stievano, P. Manfredi, and F. Canavero, "Carbon nanotube interconnects: Process variation via polynomial chaos," IEEE Trans. Electromagn. Compat., vol. 54, no. 1, pp. $140-148$, Feb. 2012.

[6] R. Pulch, "Stochastic collocation and stochastic galerkin methods for linear differential algebraic equations," Journal of Computational and Applied Mathematics, 2012.

[7] H. C. Elman, C. W. Miller, E. T. Phipps, and R. S. Tuminaro4, "Assessment of collocation and galerkin approaches to linear diffusion equations with random data," International Journal for Uncertainty Quantification, vol. 1, no. 1, pp. 19-33, 2011.

[8] T.-A. Pham, E. Gad, M. Nakhla, and R. Achar, "Efficient hermite-based variability analysis using approximate decoupling technique," in Signal and Power Integrity (SPI), 2013 IEEE 17th Workshop on, 2013, pp. 111-114. 
[9] M. H. Kalos and P. A. Whitlock, The Monte Carlo Methods, 2nd ed. NJ:Wiley, 2008.

[10] M. Evans, N. Hastings, and B. Peacock, Statistical Distributions, 3rd ed. New York: Wiley, 2000.

[11] N. Wienner, "The homogeneous chaos," American Journal of Mathematics, vol. 60, no. 4, pp. 897-936, Oct. 1938.

[12] R. H. Cameron and W. T. Martin, "The orthogonal development of non-linear functionals in series of fourier-hermite functionals," Annals of Mathematics, Second Series, vol. 48, no. 2, pp. 385-392, Apr. 1947.

[13] M. Abramowitz and I. Stegun, Handbook of Mathematical Functions. Washington, D.C.: National Bureau of Standards, Applied Mathematics Series 55, 1964.

[14] M. S. Eldred, "Recent Advances in Non-Intrusive Polynomial Chaos and Stochastic Collocation Methods for Uncertainty Analysis and Design," in Structures, Structural Dynamics, and Materials Conference. Sandia National Laboratories†, Albuquerque, NM 87185, May 2009. [Online]. Available: http://dakota.sandia.gov/papers/AIAA-2009-2274.pdf

[15] C. F. Dunkl and Y. Xu, Orthogonal Polynomials of Several Variables, ser. Encyclopedia of Mathematics and its Applications. Cambridge, 2001.

[16] C.-W. Ho, A. Ruehli, and P. Brennan, "The modified nodal approach to network analysis," IEEE Trans. Circ. and Sys., vol. 22, no. 6, pp. 504 - 509, Jun. 1975.

[17] D. Xiu, "Fast numerical methods for stochastic computations: a review," Communications in Computational Physics, vol. 5, no. 2-4, pp. 242-272, Feb. 2009.

[18] I. Gradshteyn and I. Ryzhik, Table of integrals, series and products. Acadmic Press, 2007.

[19] I. S. Stievano and F. G. Canavero, "Response variability of high-speed interconnects via hermite polynomial chaos," in Signal Propagation on Interconnects (SPI), 2010 IEEE 14th Workshop on, May 2010, pp. 3 -6.

[20] D. Bernstein, Matrix Mathematics. Theory, Facts, and Formulas with Applications to Linear Systems Theory. Princeton University Press, 2005. 
[21] J. Vlach and K. Singhal, Computer Methods for Circuit Analysis and Design. New York: Van Nostrand Reinhold, 1983.

[22] Y. Liu, L. T. Pileggi, and A. J. Strojwas, "Model order reduction of rc(l) interconnect including variational analysis," in Proc. IEEE/ACM Design Automation Conf., 1999, pp. 201-206.

[23] J. M. Wang, O. Hafiz, and J. Li, "A linear fractional transform (lft) based model for interconnect parametric uncertainty," in Proc. of 41 st Design Automation Conf., 2004, pp. 375-380.

[24] P. Heydari and M. Pedram, "Model reduction of variable-geometry interconnects using variational spectrally-weighted balanced truncation," in Proc. IEEE/ACM Intl. Conf. Computer-Aided Design, 2001, pp. 586-591.

[25] Y. Zou, Y. Cai, Q. Zhou, X. Hong, S. X.-D. Tan, and L. Kang, "Practical implementation of stochastic parametrized model order reduction via hermite polynomial chaos," in 2007 Asia and South Pacific Conference on Design Automation, 2007, pp. 367-372.

[26] L. T. Pillage and R. A. Rohrer, "Asymptotic waveform evaluation for timing analysis," IEEE Trans. Computer-Aided Design of Integrated Circ. Sys., vol. 9, pp. 352-366, Apr. 1990.

[27] E. Chiprout and M. Nakhla, Asymptotic Waveform Evaluation and Moment Matching for Interconnect Analysis. Boston: Kluwer, 1993.

[28] G. A. Baker Jr., Essentials of Padé Approximants. New York: Academic Press, 1975.

[29] E. Chiprout and M. Nakhla, "Analysis of interconnect networks using complex frequency hopping (CFH)," IEEE Trans. Computer-Aided Design of Integrated Circ. Sys., vol. 14, pp. 186-200, Feb. 1995.

[30] M. Sylveria, M. Kamon, I. Elfadel, and J. White, "A coordinate-transformed Arnoldi algorithm for generating guaranteed stable reduced-order models of arbitrary RLC circuits," in Proc. IEEE/ACM Intl. Conf. Computer-Aided Design, Nov. 1996, pp. 288-294.

[31] I. M. Elfadel and D. D. Ling, "A block rational arnoldi algorithm for multipoint passive model-order reduction of multiport RLC networks," in Proc. IEEE/ACM Intl. Conf. Computer-Aided Design, 1997, pp. 66-71. 
[32] K. J. Kerns and A. T. Yang, "Preservation of passivity during RLC network network reduction via split congruence transformations," IEEE Trans. ComputerAided Design of Integrated Circ. Sys., vol. 17, pp. 582-591, Jul. 1998.

[33] A. Odabasioglu, M. Celik, and L. T. Pileggi, "PRIMA: passive reduced-order interconnect macromodeling algorithm," IEEE Trans. Computer-Aided Design of Integrated Circ. Sys., vol. 17, no. 8, pp. 645-654, Aug. 1998.

[34] R. Achar and M. Nakhla, "Simulation of high-speed interconnects," Proceedings of IEEE, vol. 89, no. 5, pp. 693-728, May 2001. 